\title{
Diagnosing Long-QT Syndrome, Simple but not easy
}

Citation for published version (APA):

Hermans, B. J. M. (2020). Diagnosing Long-QT Syndrome, Simple but not easy. [Doctoral Thesis, Maastricht University]. Gildeprint en Universitaire Pers Maastricht. https://doi.org/10.26481/dis.20200110bh

Document status and date:

Published: 01/01/2020

DOI:

10.26481/dis.20200110bh

Document Version:

Publisher's PDF, also known as Version of record

\section{Please check the document version of this publication:}

- A submitted manuscript is the version of the article upon submission and before peer-review. There can be important differences between the submitted version and the official published version of record.

People interested in the research are advised to contact the author for the final version of the publication, or visit the DOI to the publisher's website.

- The final author version and the galley proof are versions of the publication after peer review.

- The final published version features the final layout of the paper including the volume, issue and page numbers.

Link to publication

\footnotetext{
General rights rights.

- You may freely distribute the URL identifying the publication in the public portal. please follow below link for the End User Agreement:

www.umlib.nl/taverne-license

Take down policy

If you believe that this document breaches copyright please contact us at:

repository@maastrichtuniversity.nl

providing details and we will investigate your claim.
}

Copyright and moral rights for the publications made accessible in the public portal are retained by the authors and/or other copyright owners and it is a condition of accessing publications that users recognise and abide by the legal requirements associated with these

- Users may download and print one copy of any publication from the public portal for the purpose of private study or research.

- You may not further distribute the material or use it for any profit-making activity or commercial gain

If the publication is distributed under the terms of Article $25 \mathrm{fa}$ of the Dutch Copyright Act, indicated by the "Taverne" license above, 


\section{DIAGNOSING \\ LONG-QT SYNDROME, \\ Simple but nat Easy}




\title{
DIAGNOSING LONG-QT SYNDROME, \\ Simple but nat Easy
}

\author{
Proefschrift \\ ter verkrijging van de graad van doctor aan de Universiteit Maastricht, \\ op gezag van de Rector Magnificus, prof. dr. Rianne M. Letschert, \\ volgens het besluit van het College van Decanen, \\ in het openbaar te verdedigen \\ op vrijdag 10 januari 2020 om 12:00 uur.
}


Promotores

Prof. dr. T. Delhaas

Prof. dr. L.A.F.G. Pison (Universiteit Hasselt)

Copromotor

Dr. P.G. Postema (Amsterdam UMC)

\section{Beoordelingscommissi}

Prof. dr. P.G.A. Volders (voorzitter)

Prof. dr. N.A. Blom (Amsterdam UMC)

Prof. dr. F.W. Prinzen

Prof. dr. M.A. Vos (UMC Utrecht)

Dr. S. Zeemering

\section{Table of Contents}

Chapter 1

Chapter 2 The Development and Validation of an Easy to Use Automatic QT-interval Algorithm

Chapter 3 A Comparison of the QTC-interval between Genotype-Negative LQTS Family Members and Healthy Controls

Chapter 4

QT-interval Dynamics during Brisk Standing Tests in Adult Long-QT Syndrome Patients

Chapter 5

Diagnostic Accuracy of the Response of the QT-interval to Brief Tachycardia Provoked by Standing in Children Suspect for Long-QT Syndrome

Chapter 6 Support Vector Machine-Based Assessment of the T-wave Morphology Improves LQTS Diagnosis

Chapter $7 \quad$ Improving LQTS Diagnosis by a Polynomial-Based T-wave Morphology Characterization

Chapter $8 \quad$ The Impact of Pulmonary Vein Isolation on the QT-interva

Chapter $9 \quad$ Effects of Different Dialysate Calcium Concentrations and Citric-Acid Dialysate on QTC

Chapter $10 \quad$ General Discussion

Appendices

$\begin{array}{ll}\text { A } & \text { Summary } \\ \text { B } & \text { Valorization } \\ \text { C } & \text { Dankwoord / Acknowledgements } \\ \text { D } & \text { Curriculum Vitae }\end{array}$




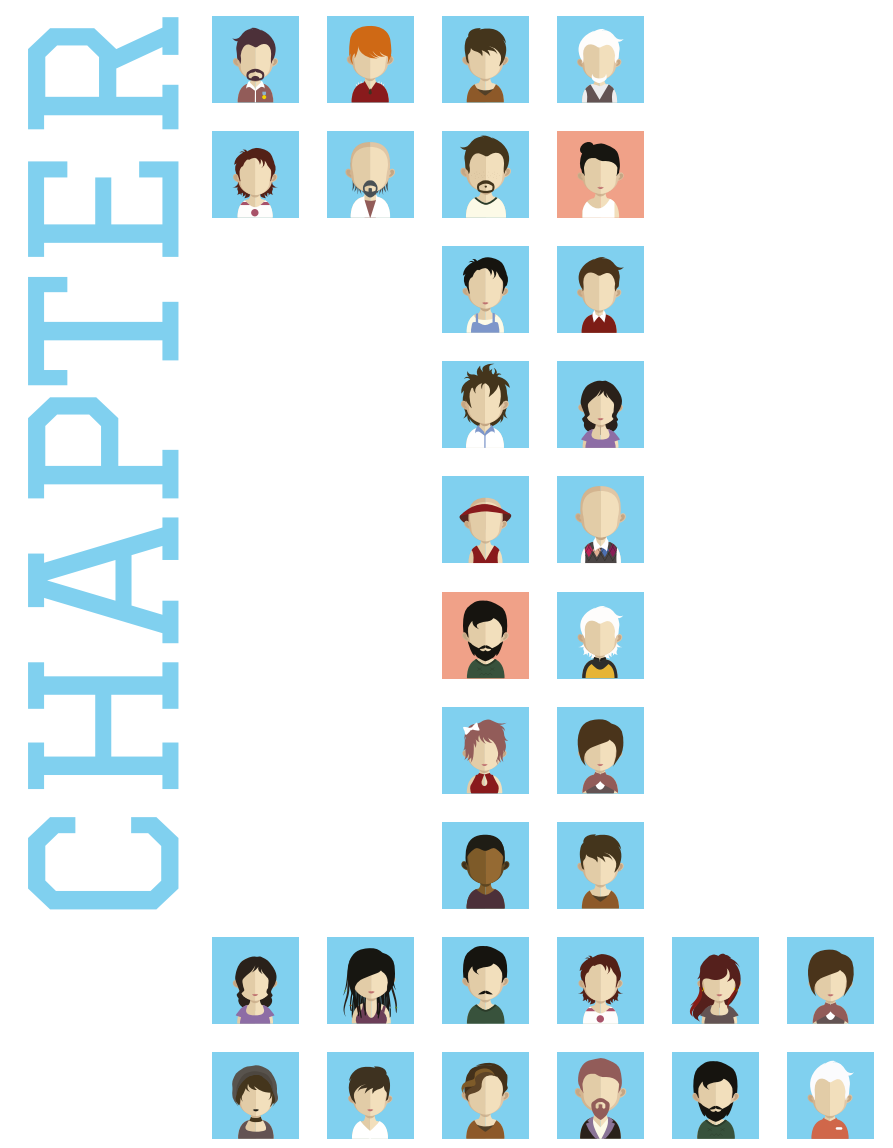

\section{CHAPTER 1}

GENERAL INTRODUCTION 


\subsection{Background}

The congenital long-QT syndrome (LQTS) is an inherited cardiac syndrome in which ventricular repolarisation is altered due to genetic mutations in genes encoding ion channels involved in the repolarization phase of the action potential. Nowadays, mutations in at least 16 genes have been found in patients with congenital LQTS'. The most common LQTS genes (KCNQ1, KCNH2 and SCN5A) account for the vast majority of all LQTS genotype-positive cases ${ }^{2,3}$. Mutations in the KCNQ1 and KCNH2 genes can lead to an altered $\mathrm{K}^{+}$current whereas mutations in the SCN5A gene can lead to an increased $\mathrm{Na}^{2+}$ inward current. Both lead to a prolonged action potential duration which is typically characterized by an increased QT-interval and/or an abnormal T-wave morphology on the electrocardiogram (ECG).

Diagnosing LQTS as soon as possible is crucial since $26 \%$ of untreated symptomatic LQTS patients will have a lethal cardiac event within three years ${ }^{4}$. If recognized and treated early enough, mortality rate drops to approximately $1 \%$ over a 15 -year follow-up 5 . Since LQTS is an inherited syndrome, genetic testing for known LQTS mutations might sound as the preferred diagnostic tool, however approximately $20 \%$ of clinically diagnosed LQTS patients remain genetically elusive ${ }^{2}$. Furthermore, the known pathogenic variants seem to have a reduced penetrance and therefore do not always lead to symptoms and/or a prolonged QT-interval6,7. Since LQTS owes its name to a prolonged QT-interval, it seems obvious to diagnose LQTS based on the QT-interval. However, diagnosing LQTS solely based on the QT-interval, even if corrected for heart rate (QTC), comes with serious limitations.

First of all, most physicians seem to be unable to identify a prolonged QT-interval when they encounte one ${ }^{8}$. Reasons for this could be erroneous QT-interval measurements, incorrect QTc calculations or the use of wrong QTc thresholds. Thereafter, two often used methods to manually assess the QTinterval (the threshold and the tangent method) lead to significantly different QT-intervals and result in a different sensitivity and specificity9. Thresholds should therefore differ for the used method. An accurate computer algorithm which determines the QT-interval would be a solution for the measuremen problems and can put an end to the method-specific thresholds if the same algorithm is used worldwide. Unfortunately, the accuracy of the existing algorithms is doubted by worldwide LQTS experts ${ }^{10}$.

Secondly, the considerable overlap in QTc obtained from standard resting ECGs between affected and unaffected individuals ${ }^{11}$ hampers screening for LQTS based on standard ECGs. Therefore, instead of studying QTc at rest, provocative tests have been introduced to study the capability of the QT-interval to adjust to changes in heart rate accomplished by epinephrine ${ }^{12,13}$, exercise ${ }^{14,15}$ or brisk standing ${ }^{16-18}$. Though these studies report improved diagnosis of LQTS, the suspicion for LQTS has to be raised before these tests will be used.

Finally, the QT-interval is effected by age, gender and hormones making it unstable over time ${ }^{19}$. LQTS patients can show a prolonged QT-interval on one ECG and a normal on a repeated $\mathrm{ECG}^{20}$. Considering all limitations mentioned above, Diagnosing Long-QT Syndrome: Simple but not so Easy.

\subsection{General Aim}

In this thesis, we aim to improve the diagnosis of congenital LQTS based on computational analysis of the ECG. We hypothesize that the ECG contains more diagnostic information regarding LQTS than is 
used in clinical practice. Therefore, we developed, analyzed and applied various algorithms which might improve the diagnosis of LQTS.

\subsection{Outline of Thesis}

To solve the problems with measuring the QT-interval, we introduce a fully automatic algorithm to measure QT-intervals in Chapter 2 of this thesis. The algorithm is based on the tangent method to measure the end of the T-wave ${ }^{21}$. Apart from a detailed description, the algorithm is validated by a direct comparison of QT-intervals measured by the algorithm and as measured by three observers.

Other genetic factors distinct from the LQTS mutation can influence the expression of the LQTS mutation ${ }^{22-24}$. Genetic testing of relatives in family screening only test for known LQTS mutations. A genotype-negative family member can therefore still have an altered repolarization caused by other mutations. Several studies used genotype-negative family members as controls whereas others use healthy subjects as controls. Whether the definition of the control group influences the results of these studies is unknown. Therefore, in Chapter $\mathbf{3}$ we investigate whether genotype-negative family members have a prolonged QTC with respect to healthy controls.

In Chapter 4 we applied the automatic algorithm described in Chapter 2 to ECGs recorded during supine-standing tests to study the dynamic behaviour of the QTc and QT-interval in LQTS patients and genotype-negative family members. The so far promising results of the supine-standing test rely on manually assessed QT-intervals of several predefined complexes ${ }^{16-18}$. In this chapter we examine the diagnostic value of the QT-intervals of these complexes as well as the dynamic behaviour of the QTC and QT-intervals of all complexes in adult LQTS patients and controls. We furthermore investigate sexdifferences in the response to the supine-standing test.

Since children not only have a higher heart rate at rest but also have a more pronounced reflex tachycardia ${ }^{25}$, the promising results of the supine-standing tests in adults can't be extrapolated to paediatric cohort. Therefore, in Chapter $\mathbf{5}$ we study the usefulness of the supine-standing test in a paediatric cohort of healthy children and children with LQTS. Since this is the first supine-standing test study on a paediatric cohort including LQTS patients, we also study the reproducibility of the test on this cohort.

Apart from the prolonged QT-interval, altered T-wave morphologies have also been recognized in LQTS patients ${ }^{26,27}$. In Chapter 6 and Chapter 7 we study whether the T-wave in a standard 10-second resting ECG can aid in the diagnosis of LQTS. In Chapter 6 we use various known T-wave morphology parameters and combine these in a support vector machine model to improve LQTS diagnosis. In Chapter 7 we introduce a new polynomial-based method to characterize the T-wave morphology and study the additional value of this characterization in the diagnosis of LQTS.

A prolonged QT-interval is not only seen in LQTS patients. Other pathologies (e.g. ischemia, ionic mbalance) or treatments (e.g. drug-induced LQTS) can also lead to a prolonged QT-interval. Acquired prolonged QT-intervals are also associated with ventricular arrhythmias and therefore also need to be diagnosed as soon as possible. In Chapter $\mathbf{8}$ and Chapter $\mathbf{9}$, we study two interventions that might lead to a prolonged QT-interval. In Chapter $\mathbf{8}$ we study the effects of pulmonary vein isolation on the
QT-interval. As a consequence of a collateral damage occurring during isolation of the pulmonary veins, ganglionated plexi located outside of the left atrium may be modulated ${ }^{28}$. Recent studies have shown that this might also affect ventricular electrophysiology ${ }^{29,30}$. For example, an experimental study in canine hearts showed that the ventricular action potential duration significantly increased after ablating the ganglionated plexi. Therefore, in Chapter $\mathbf{8}$ we study whether the QT-interval (i.e. the ventricular action potential duration) prolongs as a consequence of pulmonary vein isolation. In Chapter $\mathbf{9}$ we study the effect of various calcium concentrations in dialysate on the QT-interval. Calcium is an important electrolyte in the repolarization of cardiac myocytes. Different calcium concentrations in dialysates might therefore affect the ventricular repolarization and lead to a prolonged QT-interval.

In the final chapter of this thesis, Chapter 10, the findings of the various chapters are put into broader perspective in a general discussion. 


\subsection{References}

1. Schwartz, P. J., Ackerman, M. J., George, A. L. \& Wilde, A. A. M. Impact of genetics on the clinical management of channelopathies. J. Am. Coll. Cardiol. 62, 169-180 (2013).

2. Kapplinger, J. D. et al. Spectrum and prevalence of mutations from the first 2,500 consecutive unrelated patients referred for the FAMILION® long QT syndrome genetic test. Heart Rhythm 6, 1297-1303 (2009).

3. Tester, D. J., Will, M. L., Haglund, C. M. \& Ackerman, M. J. Compendium of cardiac channel mutations in 541 consecutive unrelated patients referred for long QT syndrome genetic testing. Heart Rhythm 2, 507-517 (2005).

4. Schwartz, P. J. Idiopathic long QT syndrome: Progress and questions. Am. Heart J. 109, 399-411 (1985)

5. Schwartz, P. J. \& Crotti, L. Cardiac Electrophysiology: From Cell to Bedside. (Elsevier/Saunders, 2009).

6. Crotti, L. et al. The common long-QT syndrome mutation KCNQ1/A341V causes unusually severe clinical manifestations in patients with different ethnic backgrounds: Toward a mutation-specific risk stratification. Circulation 116, 2366-2375 (2007).

7. Priori, S. G., Napolitano, C. \& Schwartz, P. J. Low penetrance in the long-QT syndrome: clinica impact. Circulation 99, 529-533 (1999)

8. Viskin, S. et al. Inaccurate electrocardiographic interpretation of long QT: The majority of physicians cannot recognize a long QT when they see one. Heart Rhythm 2, 569-574 (2005).

9. Vink, A. S. et al. Determination and Interpretation of the QT Interval. Circulation 138, 2345-2358 (2018).

10. Taggart, N. W., Haglund, C. M., Tester, D. J. \& Ackerman, M. J. Diagnostic miscues in congenital longQT syndrome. Circulation 115, 2613-2620 (2007).

11. Viskin, S. The QT interval: Too long, too short or just right. Heart Rhythm 6, 711-715 (2009).

12. Vyas, H., Hejlik, J. \& Ackerman, M. J. Epinephrine QT Stress Testing in the Evaluation of Congenital Long-QT Syndrome. Circulation 113, 1385-1392 (2006).

13. Shimizu, W. et al. Diagnostic value of epinephrine test for genotyping LQT1, LQT2, and LQT3 forms of congenital long QT syndrome. Heart Rhythm 1, 276-283 (2004)

14. Sy, R. W. et al. Derivation and validation of a simple exercise-based algorithm for prediction of genetic testing in relatives of LQTS probands. Circulation 124, 2187-2194 (2011).

15. Horner, J. M., Horner, M. M. \& Ackerman, M. J. The diagnostic utility of recovery phase QTc during treadmill exercise stress testing in the evaluation of long QT syndrome. Heart Rhythm 8, 16981704 (2011)

16. Viskin, S. et al. The Response of the QT Interval to the Brief Tachycardia Provoked by Standing. J. Am Coll. Cardiol. 55, 1955-1961 (2010).

17. Chorin, E. et al. Diagnostic value of T-wave morphology changes during "QT stretching" in patients with long QT syndrome. Heart Rhythm 12, 2263-2271 (2015).

18. Adler, A. et al. The phenomenon of "QT stunning": The abnormal QT prolongation provoked by standing persists even as the heart rate returns to normal in patients with long QT syndrome. Heart Rhythm 9, 901-908 (2012).

19. Vink, A. S., Clur, S. A. B., Wilde, A. A. M. \& Blom, N. A. Effect of age and gender on the QTc-interval in healthy individuals and patients with long-QT syndrome. Trends Cardiovasc. Med. 28, 64-75 (2018)

20. Hoefen, R. et al. In silico cardiac risk assessment in patients with long QT syndrome: Type 1: Clinical predictability of cardiac models. J. Am. Coll. Cardiol. 60, 2182-2191 (2012).
21. Postema, P. G., De Jong, J. S. S. G., Van der Bilt, I. A. C. \& Wilde, A. A. M. Accurate electrocardiographic assessment of the QT interval: Teach the tangent. Heart Rhythm 5, 1015-1018 (2008).

22. Crotti, L. et al. KCNH2-K897T is a genetic modifier of latent congenital long-QT syndrome. Circulation $112,1251-1258(2005)$

23. Brink, P. A. et al. Phenotypic variability and unusual clinical severity of congenital long-QT syndrome in a founder population. Circulation 112, 2602-2610 (2005).

24. Tomás, M. et al. Polymorphisms in the NOS1AP Gene Modulate QT Interval Duration and Risk of Arrhythmias in the Long QT Syndrome. J. Am. Coll. Cardiol. 55, 2745-2752 (2010).

25. Ives, C. T. \& Kimpinski, K. Higher postural heart rate increments on head-up tilt correlate with younger age but not orthostatic symptoms. J. Appl. Physiol. 115, 525-528 (2013).

26. Lehmann, M. H. et al. T wave 'humps' as a potential electrocardiographic marker of the long QT syndrome. J. Am. Coll. Cardiol. 24, 746-754 (1994).

27. Moss, A. J. et al. ECG T-Wave Patterns in Genetically Distinct Forms of the Hereditary Long QT Syndrome. Circulation 92, 2929-2934 (1995).

28. Pappone, C. et al. Pulmonary Vein Denervation Enhances Long-Term Benefit after Circumferentia Ablation for Paroxysmal Atrial Fibrillation. Circulation 109, 327-334 (2004).

29. Jungen, C. et al. Disruption of cardiac cholinergic neurons enhances susceptibility to ventricular arrhythmias. Nat. Commun. 8, (2017)

30. He, B. et al. Effects of ganglionated plexi ablation on ventricular electrophysiological properties in normal hearts and after acute myocardial ischemia. Int. J. Cardiol. 168, 86-93 (2013). 


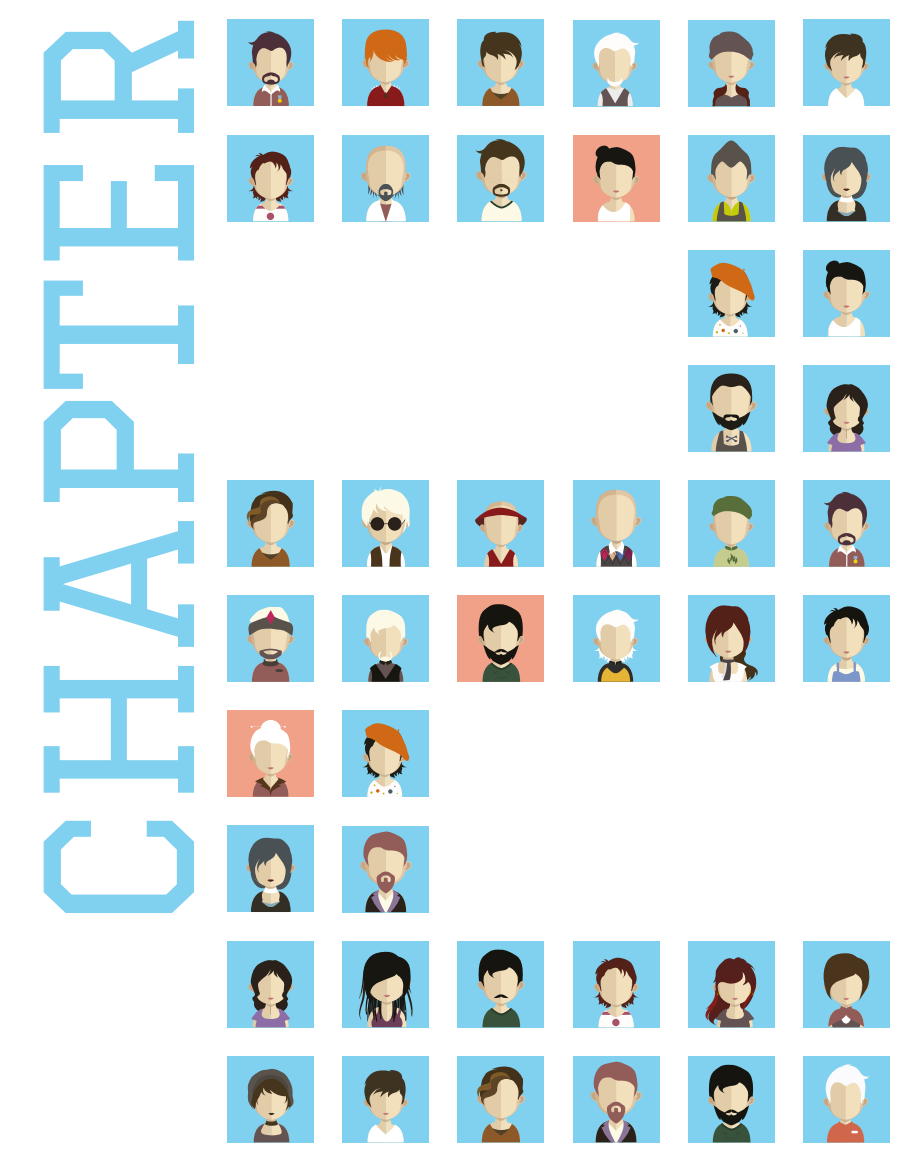

\section{CHAPTER 2}

THE DEVELOPMENT AND VALIDATION OF AN EASY TO USE AUTOMATIC QT-INTERVAL ALGORITHM 
Abstract

\section{Background}

To evaluate QT-interval dynamics in patients and in drug safety analysis, beat-to-beat QT-interval measurements are increasingly used. However, interobserver differences, aberrant T-wave morphologies and changes in heart axis might hamper accurate QT-interval measurements.

Objective

To develop and validate a QT-interval algorithm robust to heart axis orientation and T-wave morphology that can be applied on a beat-to-beat basis.

Methods

Additionally to standard ECG leads, the root mean square $\left(\mathrm{ECG}_{\mathrm{RMS}}\right)$, standard deviation and vectorcardiogram were used. QRS-onset was defined from the $E_{C G_{R M S}}$. T-wave end was defined per ndividual lead and scalar ECG using an automated tangent method. A median of all T-wave ends was used as the general T-wave end per beat.

Supine-standing tests of 73 patients with Long-QT syndrome (LQTS) and 54 controls were used because they have wide ranges of RR and QT-intervals as well as changes in T-wave morphology and heart axis orientation. For each subject, QT-intervals in three random complexes chosen from the low, middle and high RR range, were compared with manually measurements by three observers.

\section{Results}

After visual inspection of the randomly selected complexes, 21 complexes were excluded because of evident noise, too flat T-waves or premature ventricular beats. Bland-Altman analyses of automatically and manually determined QT-intervals showed a bias of $<4 \mathrm{~ms}$ and limits of agreement of $\pm 25 \mathrm{~ms}$. Intra-class coefficient indicated excellent agreement $(>0.9)$ between the algorithm and the observers.

\section{Conclusion}

Our automated algorithm provides reliable beat-to-beat QT-interval assessment, robust to heart axis and T-wave morphology.

\subsection{Introduction}

Prolongation of the QT-interval on the electrocardiogram (ECG) has been associated with Torsade de Pointes, a potentially lethal cardiac arrhythmia',2. A prolonged QT-interval can be caused by Long-Q syndrome (LQTS), which can be either inherited or acquired due to an underlying medical condition or medication ${ }^{2}$. The measurement of the QT-interval is used world-wide on a daily basis in the diagnosis of LQTS or in the evaluation of possible effects of a new drug on the QT-interval

Although the value of a prolonged QT-interval for risk assessment of future malignant arrhythmias is widely understood', most physicians, including cardiologists, have difficulties to correctly identify a prolonged QT-interval ${ }^{4}$. Additionally to measurement difficulties, diagnosing LQTS is challenging since there is a considerable overlap of the QT-interval between LQTS patients and healthy controls. ${ }^{5,6}$ Because of this overlap in QT-intervals, additional measurements like QT dispersion ${ }^{7,8}$ and QT variability ${ }^{9}$ were introduced and assessed on their value to diagnose LQTS. Because these relatively new parameters are used to study QT dynamics, they require evaluation of large numbers of RR- and QT-intervals. QT variability, for example, is typically determined from 256-512 beats or 256-512 seconds duration ECG9. Furthermore, supine-standing tests are introduced to study QT-interval adaptation ${ }^{10,11}$ and changes in T-wave morphologies ${ }^{12}$ due to heart rate changes induced by brisk standing. In these tests, QT-interva dynamics are assessed based on a small number of QT-intervals ${ }^{10,11}$. Beat-to-beat analysis of supinestanding tests might give more insight in the dynamic behaviour of the QT-interval and therefore improve its diagnostic value. Measuring these large numbers of RR- and QT-intervals manually is very time consuming and therefore automated QT-interval algorithms are necessary.

Currently, automatic algorithms for measuring the QT-interval embedded in commercial ECG systems measure the QT-interval on an average or median complex over time (cf. Appendix Kligfield et al. ${ }^{13}$ ). As a consequence, beat-to-beat detection algorithms which include the QT-interval dynamicity have been published, but often use only a single ECG lead (mostly II or V5), which makes the QT-interval susceptible to heart axis orientation and electrode placement ${ }^{14}$. In this article we present and validate an automatic QT-interval algorithm based on the tangent method ${ }^{15}$ which is unaffected by heart axis orientation and that can be applied on a beat-to-beat basis regardless of the T-wave morphology.

\subsection{Methods}

\subsubsection{Population and ECG recordings}

Five minutes long ECGs from supine-standing tests recorded between December 2008 and February 2016 of 73 LQTS patients and 54 controls were included in this study. These recordings were performed in the initial evaluation of individuals referred to the department of Cardiology and Cardiogenetics of the Academic Medical Centre in Amsterdam, The Netherlands, in the work-up during family screening for LQTS (i.e. after a diagnosis was made in an index patient). LQTS patients had a confirmed pathogenic mutation in either the KCNQ1, KCNH2 or SCN5A gene resulting in LQTS type 1 (LQT1), type 2 (LQT2) o type 3 (LQT3) respectively. Controls were genotype-negative family members or healthy volunteers. We obtained a waiver from the local ethical committee for ethical approval for the conduct of this study. 


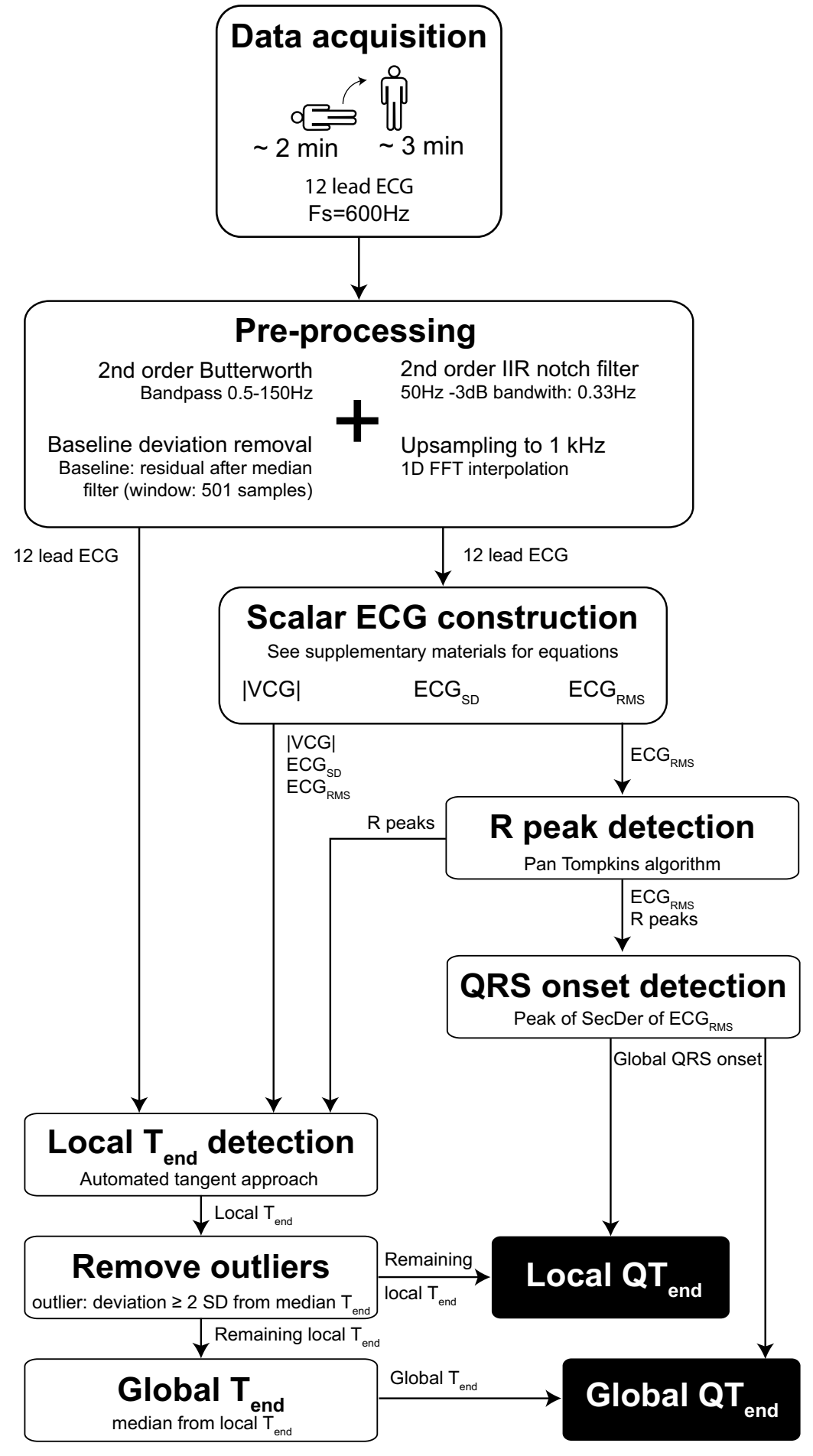

Figure 2-1 Schematic representation the algorithm's steps. A detailed description is given in the main text. Fs = sample frequency, $\mathrm{Hz}=$ hertz, $\| \mathrm{R}=$ infinite impulse response, $\mathrm{FFT}=$ fast Fourier transform, $\mid$ VCG $\mid$ = magnitude of the vectorcardiogram, SD = standard deviation, RMS = root mean square, SecDer = second derivative, $T_{\text {end }}=T$ wave end.

ECG recordings during supine-standing tests were used to validate the algorithm since these recordings consist of a wide range of RR- and QT-intervals as well as changes in T-wave morphology and heart axis orientation $10-12$

\subsubsection{Development of an automatic QT-interval detection algorithm}

\section{Data acquisition and pre-processing}

All individual (pre-)processing steps of the algorithm are shown in Figure 2-1. All ECGs were recorded with a $600 \mathrm{~Hz}$ sample frequency using Welch Allyn CardioPerfect (Welch Allyn, Skaneateles Falls, NY, USA) Data analysis was performed offline using a custom-made Matlab (2015b, The MathWorks, Natick, MA, USA) program. After acquisition, ECG data were filtered using a 2nd order bidirectional Butterworth band pass filter $\left(0.5-100 \mathrm{~Hz}^{16}\right)$ and a 2 nd order infinite impulse response notch filter $(50 \mathrm{~Hz})$ with a $-3 \mathrm{~dB}$ bandwidth of $0.33 \mathrm{~Hz}$. For all individual leads, the residuals of a median filter with a 501 samples window were regarded as baseline deviations and were subtracted from the individual ECG leads to correct for baseline wander. The filtered ECGs were thereafter upsampled to $1000 \mathrm{~Hz}$ to make the analysis sample frequency independent so it can be applied to ECGs recorded with different sample frequencies as well.

\section{Scalar ECG construction}

Three types of scalar ECGs are constructed to emulate ECG signals unaffected by heart axis orientation. The root mean square ( $\left.\mathrm{ECG}_{\mathrm{pus}}\right)$ and standard deviation $\left(\mathrm{ECG}_{\mathrm{SD}}\right)$ are calculated as follows:

$\operatorname{ECG}_{\mathrm{RMS}}(t)=\sqrt{\frac{1}{9} \sum_{i=1}^{9} \operatorname{ECG}_{i}^{2}(t)}$

$\mathrm{ECG}_{\mathrm{SD}}(t)=\sqrt{\frac{1}{9} \sum_{i=1}^{9}\left(\mathrm{ECG}_{i}(t)-\overline{\mathrm{ECG}}(t)\right)^{2}}$

where $\mathrm{ECG}_{.}(\mathrm{t})$ is the ECG signal at time $t$ from lead $i$ and $\overline{\mathrm{ECG}}(t)$ is the mean $\mathrm{ECG}$ in time over the various leads. Note that there are only nine leads used in this calculation. Ideally, one would use unipolar precordial leads and unipolar limb leads to reconstruct a scalar ECG from. Unfortunately, true unipolar limb leads are not recorded (or saved) in a standard 12-lead ECG. Mathematically, augmented limb leads are scaled true unipolar ECG leads. For example, the unipolar foot electrode (VF) would be calculated by:

$\mathrm{VF}=\Phi_{\mathrm{f}}-\Phi_{\mathrm{wct}}=\Phi_{\mathrm{f}}-\frac{\Phi_{\mathrm{f}}+\Phi_{\mathrm{r}}+\Phi_{1}}{3}=\frac{2 \Phi_{\mathrm{f}}-\Phi_{\mathrm{r}}-\Phi_{1}}{3}$

with $\Phi_{f^{\prime}} \Phi_{r}$ and $\Phi_{1}$ the potential recorded at the foot, right arm and left arm respectively and $\Phi_{\text {wct }}$ the Wilson central terminal. 
The augmented limb lead aVF is calculated by:

$\mathrm{aVF}=\Phi_{\mathrm{f}}-\frac{\Phi_{\mathrm{r}}+\Phi_{1}}{2}=\frac{2 \Phi_{\mathrm{f}}-\Phi_{\mathrm{r}}-\Phi_{1}}{2}$

So, VF can be calculated from aVF by scaling aVF with 2/3.

$\frac{2}{3} \cdot \frac{\left(2 \Phi_{\mathrm{f}}-\Phi_{\mathrm{r}}-\Phi_{1}\right)}{2}=\frac{2 \Phi_{\mathrm{f}}-\Phi_{\mathrm{r}}-\Phi_{1}}{3}$

We used these calculated unipolar limb leads (2/3 aVR, 2/3 aVL, 2/3 aVF) and the unipolar precordial leads (V1-V6) to construct the $\mathrm{ECG}_{\mathrm{RMS}}$ and $\mathrm{ECG}_{\mathrm{SP}}$

Lastly, a vectorcardiogram (VCG) was constructed using the method described by Kors et al. ${ }^{17}$ The magnitude of this VCG (|VCG |) was used as the third scalar ECG.

\section{Reak and QRS onset detection}

$R$ peaks were detected from the $\mathrm{ECG}_{\text {pus }}$ signal using the Pan Tompkins algorithm ${ }^{18}$. The largest peak in the second derivative of $E \mathrm{EG}_{\mathrm{RMS}}$ (calculated using a simple numerical differentiation) within a window of 100 to 20 milliseconds ( $\mathrm{ms}$ ) preceding the R peak was regarded to indicate the onset of the QRS complex, see Figure 2-2.

\section{T-wave landmarks}

The peak of the T-wave ( $\left.T_{\text {peak }}\right)$ and the end of the T-wave $\left(T_{\text {end }}\right)$ are estimated for every individual ECG lead as well as for the constructed scalar ECG signals. T-wave landmarks obtained from individual scalar ECG signals or ECG leads are called local T-wave landmarks. Since individual ECG leads are affected by heart axis orientation and scalar ECGs may blur information which is only present in one or two individual ECG leads, local T-wave landmarks are determined from both the individual ECG leads and the scalar ECGs. These effects are minimized by determining one global T-wave landmark from the local T-wave andmarks obtained from ECG leads and scalar ECGs. To detect the local T-wave landmarks, all individua ECG leads and the scalar ECG signals were smoothed using a 2nd order Savitzky Golay filter with a 50ms window. First, the local peak of the T-wave (local $\mathrm{T}_{\text {peak }}$ ) was detected as the maximum or minimum peak between the preceding R peak $+50 \mathrm{~ms}$ and the preceding R peak $+70 \%$ RR of the smoothed signal (see Figure 2-2). Second, the slope of the maximum deflection between local $\mathrm{T}_{\text {peak }}$ and local $\mathrm{T}_{\text {peak }}+30 \% \mathrm{RR}$ was calculated. A tangent through the point with the maximal slope in the final limb of the T-wave was estimated using a simple numerical differentiation within a ten ms window $(\mathrm{dV} d \mathrm{t}(\mathrm{t})=(\mathrm{V}(\mathrm{t}+5)-\mathrm{V}(\mathrm{t}-5)) / 10)$ The intersection of this tangent and the baseline was used to detect the local end of the T-wave (loca $T_{\text {end }}$. The baseline was defined as the median amplitude of the $30 \mathrm{~ms}$ preceding the QRS onset of that particular complex. Local QT-intervals were calculated from the global QRS onset and local $T_{\text {end }}$ and can be used for QT dispersion measures.

\section{Global T-wave landmarks}

Fom the local T-wave landmarks, a median $T_{\text {peak }}$ and $T_{\text {end }}$ location was calculated for every complex. Loca $T_{\text {peak }}$ and $\mathrm{T}_{\text {end }}$ landmarks that deviated more than two times the standard deviation (SD) from the median $T_{\text {peak }}$ and $T_{\text {end }}$ were considered to be outliers and excluded. Global $T_{\text {peak }}$ and $T_{\text {end }}$ locations were calculated as the median from the remaining local $T_{\text {peak }}$ and $T_{\text {end }}$ locations. Finally, QT-intervals were calculated by calculating the interval between QRS onset and global $T_{\text {end. }}$. Individual ECG leads with a local T-wave amplitude smaller than $50 \mu \mathrm{V}$ were considered to be too small for accurate determination of local $\mathrm{T}_{\text {end }}$ and therefore were not taken into account for the determination of the global $T$ of that particular complex. For example, if the T-wave amplitude is low in all limb leads, global $T$, will be calculated from the local $T_{\text {end }}$ of the precordial leads and the scalar ECGs only.

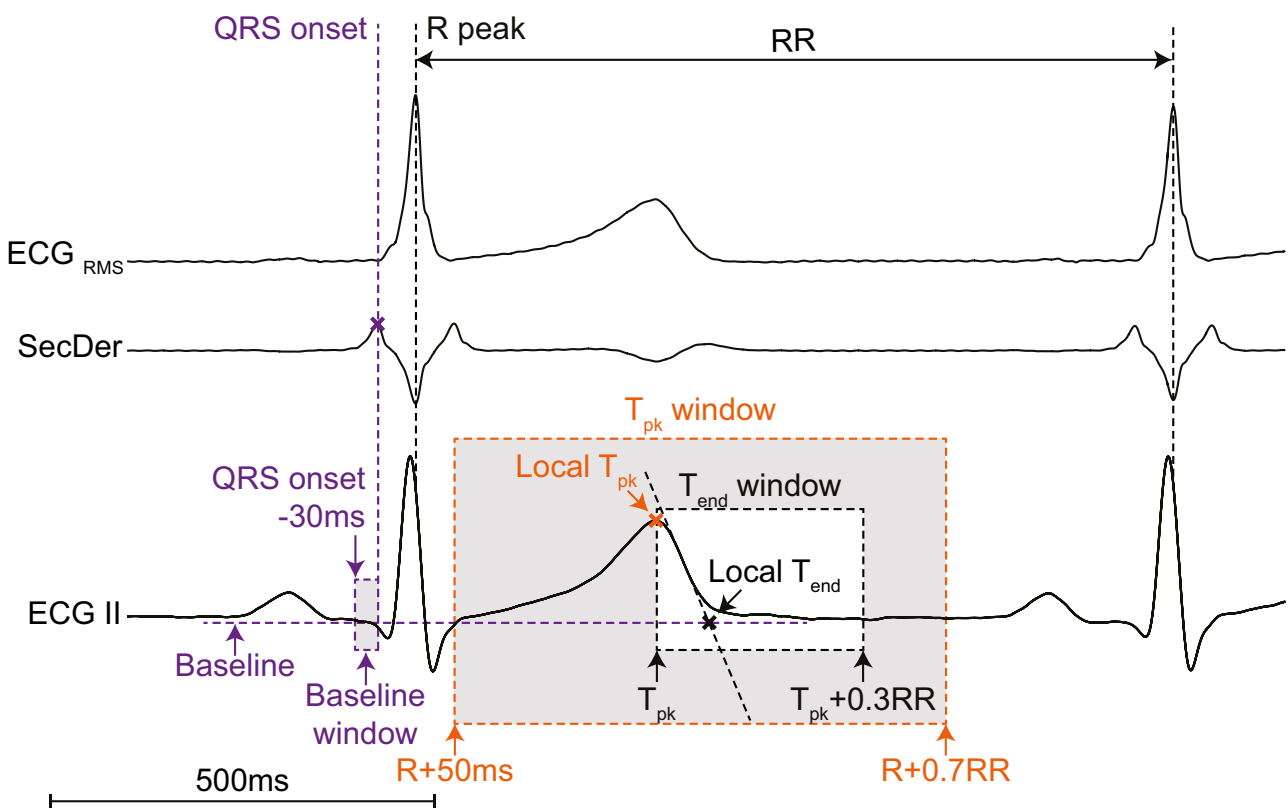

Figure 2-2 Illustration of global QRS onset and local T wave landmarks detection. Global R peak is detected using a Pan-Tompkins algorithm on the $\mathrm{ECG}_{\text {RMS }}$ signal. The global QRS onset is thereafter detected as a peak in the second derivative of the $\mathrm{ECG}_{\text {RMS }}$ within a certain window preceding the globa $\mathrm{R}$ peak. The local T peak (Tpk) is detected as the maximum or minimal peak between $\mathrm{R}+50 \mathrm{~ms}$ and $R+0.7 R R$. Thereafter, the tangent trough the point of maximum deflection between Tpk and Tpk+0.3RR is calculated from the first derivative. The intersection between this tangent and the baseline is detected as the local end of the $T$ wave $\left(T_{\text {end }}\right)$. Tpk = T-wave peak, $T_{\text {end }}=T$-wave end, RMS = root mean square, secDer = second derivative, $\mathrm{ms}=$ milliseconds.

\subsubsection{Validation}

From every ECG recording during a supine-standing test, one complex with an RR-interval below the 10th percentile, one complex with an RR-interval above the 90th percentile, and one complex with an RR-interval within the interquartile range were randomly selected by the computer. This resulted in three complexes with a wide range of RR-intervals per supine-standing test. From the randomly selected complexes, QT-intervals were calculated both automatically using the algorithm described above and manually by three independent observers (BH, FB, TD). The manual measurements of the QT-intervals were done on paper using the tangent approach in a lead of choice ${ }^{15}$. All observers measured the QTinterval with an accuracy of $0.5 \mathrm{~mm}$, which corresponded with $6 \mathrm{~ms}$. The observers were blinded for patient characteristics, QT-intervals determined by the algorithm and the measurements of the other 
observers. The algorithm was validated by determining the inter-method variability between the QTinterval measured by the algorithm (QTalg) and (I) the individual manual QT-interval measurements (QTobs1, QTobs2 and QTobs3) and (II) the mean QT-interval from all the individual measurements (HQTobs). In addition, the QT-interval measurements of the three observers were compared in order to assess the inter-observer variability.

\subsubsection{Statistical analysis}

Statistical analyses were performed in Matlab. Patient and ECG characteristics were presented in frequencies (percentage) for categorical variables and mean $( \pm S D$ ) for continuous variables with an approximately symmetric distribution. The inter-method variability and the inter-observer variability were expressed as correlation coefficients estimated by a Pearson correlation test, and the intra-class correlation coefficient (ICC) for single and averaged measurements based on a two-way mixed absolute agreement model ${ }^{19}$. Sample uncertainty was expressed as $95 \%$ confidence intervals $(95 \% \mathrm{CI})$. BlandAltman analyses were performed to assess the systematic bias and the limits of agreement for both the inter-method and the inter-observer variability ${ }^{20}$. A p-value $<0.05$ was considered to be statistically significant.

\subsection{Results}

\subsubsection{Population}

The total study population of 127 subjects included 34 (26.8\%) subjects with LQT1, 28 (22.0\%) with LQT2, $11(8.7 \%)$ LQT3, and 54 (42.5\%) controls. The characteristics of the study population are shown in Table $2-1$

\begin{tabular}{lcccccc}
\hline Gender (M/F) & Age (years) & Number (-) & $\begin{array}{l}\text { QTc at } \\
\text { low RR (ms) }\end{array}$ & $\begin{array}{l}\text { QTc at } \\
\text { mid RR (ms) }\end{array}$ & $\begin{array}{l}\text { QTc at } \\
\text { high RR (ms) }\end{array}$ \\
\hline LQT1 & $13 / 21$ & $33.9 \pm 13.9$ & 34 & $486 \pm 44$ & $460 \pm 37$ & $439 \pm 33$ \\
LQT2 & $17 / 11$ & $40.5 \pm 15.1$ & 28 & $498 \pm 49$ & $449 \pm 35$ & $427 \pm 34$ \\
\hline LQT3 & $4 / 7$ & $35.2 \pm 15.2$ & 11 & $472 \pm 45$ & $439 \pm 35$ & $422 \pm 35$ \\
Control & $31 / 23$ & $40.8 \pm 15.8$ & 54 & $446 \pm 38$ & $410 \pm 26$ & $392 \pm 25$ \\
Total & $65 / 62$ & $38.4 \pm 15.2$ & 127 & $469 \pm 47$ & $435 \pm 39$ & $415 \pm 36$
\end{tabular}

Table 2-1 Characteristics of the study population. Data are given as mean \pm standard deviation. $\mathrm{M}=$ male, $\mathrm{F}=$ female, RR = RR-interval, QTC = Corrected $\mathrm{QT}$ using Bazett's formula.

\subsubsection{Validation}

The randomly computer based selected complexes were visually inspected and eight complexes (2.1\%) had to be excluded based on the presence of artefacts, three complexes because of too flat T-waves in all ECG leads (0.8\%), and two (0.5\%) because the randomly chosen complex was a premature ventricular complex.
In the remaining 358 complexes, the RR-intervals ranged from $470 \mathrm{~ms}$ to $1419 \mathrm{~ms}$, with a mean RR of $849 \mathrm{~ms}( \pm 194 \mathrm{~ms})$. The mean heart axis was $42^{\circ}\left( \pm 42^{\circ}\right)$ and the $95 \%$ percentile confidence interval (PCI: $2.5^{\text {th }}$ and $97.5^{\text {th }}$ percentile of the data) ranged from $-45^{\circ}$ to $119^{\circ}$. The mean T-wave axis was $26^{\circ}\left( \pm 42^{\circ}\right)$ with a $95 \% \mathrm{PCl}$ ranging from $-85^{\circ}$ to $108^{\circ}$.

By visual inspection by one of the observers, 127 (35\%) complexes had aberrant T-waves and/or prominent $U$-waves. Figure 2-3 shows an example of one complex with the QRS onset and global T detected by the algorithm for an LQT1, LQT2 and LQT3 patient as well as for a control.

$\begin{array}{rcccc} & \text { Control } & \text { LQT1 } & \text { LQT2 } & \text { LQT3 } \\ \text { RR [ms] } & 962 & 1085 & 771 & 1223 \\ \text { QTalg [ms] } & 412 & 534 & 421 & 436 \\ \mu \text { QTobs [ms] } & 410 & 525 & 420 & 428\end{array}$

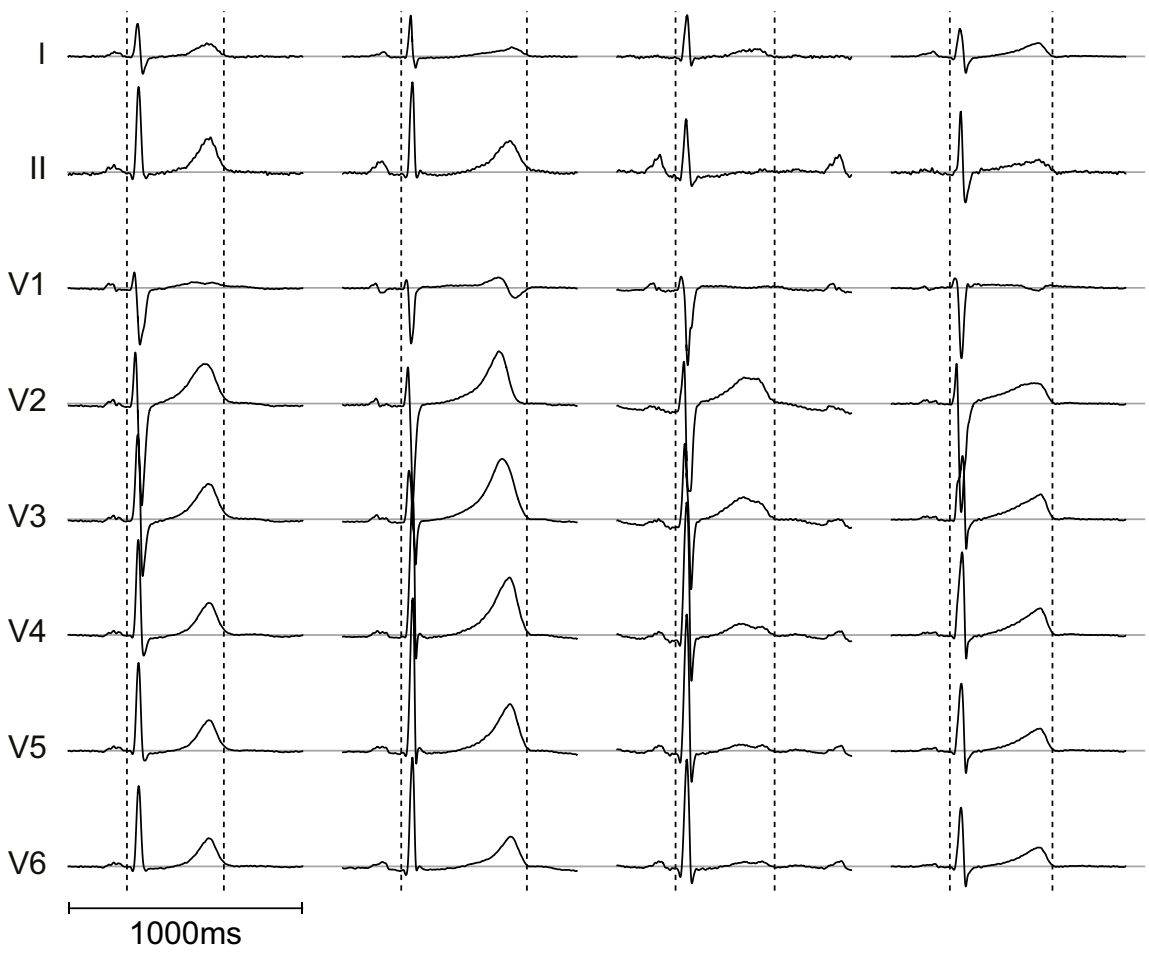

Figure 2-3 An example of the results of our algorithm. The QRS onset and global $T_{\text {end }}$ detected by the algorithm is shown for a healthy control and patients with LQT-1, 2 and 3. QTalg = QT-interval determined by the algorithm, $\mu \mathrm{QTobs}=$ mean $\mathrm{QT}$-interval determined by three observers, $\mathrm{ms}=$ milliseconds. 


\subsubsection{Inter-method variability}

Results of the comparison between the QTalg and the individual observers are shown in Table 2-2 There was a strong correlation (Pearson's $r$ ranging from 0.935 to 0.959 ) and agreement (ICC ranging from 0.933 to 0.956 ) between the QTalg and the individual observers, with a systematic bias ranging between $-1.88 \mathrm{~ms}$ and $3.39 \mathrm{~ms}$. Figure $2-4$ shows the Bland-Altman plot for the inter-method variability of QTalg and $\mu \mathrm{QT}$ Tobs. The correlation and agreement between QTalg and $\mu \mathrm{QT}$ Tobs were also strong $(r=$ 0.962, ICC $=0.981$.

LQTS type specific validation showed similar agreements between QTalg and $\mu$ QTobs for all LQTS types (ICC ranging from 0.934 (LQT2) to 0.989 (LQT1). See supporting information: Figure S.2-1 and Table S.2-1).

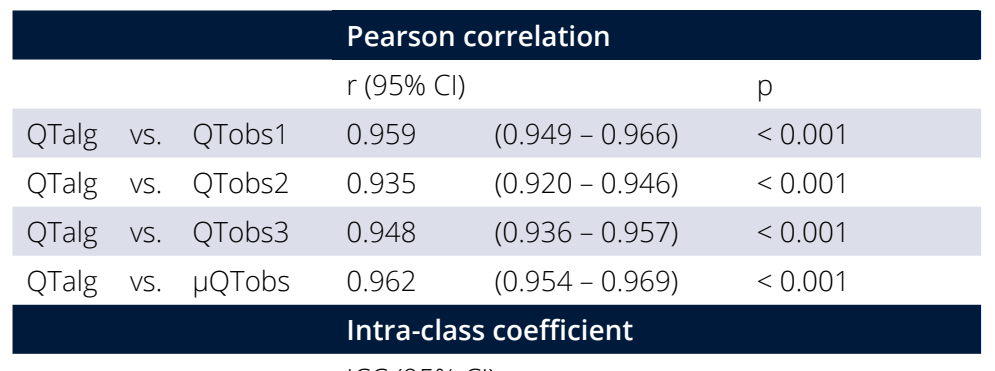

\begin{tabular}{|c|c|c|c|c|c|c|}
\hline & & & \multicolumn{3}{|c|}{ ICC (95\% CI) } & $p$ \\
\hline QTalg & vs. & QTobs1 & 0.956 & \multicolumn{2}{|c|}{$(0.943-0.966)$} & $<0.001$ \\
\hline QTalg & vs. & QTobs2 & 0.933 & \multicolumn{2}{|c|}{$(0.917-0.945)$} & $<0.001$ \\
\hline QTalg & vs. & QTobs3 & 0.947 & \multicolumn{2}{|c|}{$(0.935-0.957)$} & $<0.001$ \\
\hline QTalg & vs. & HQTobs & 0.981 & \multicolumn{2}{|c|}{$(0.976-0.984)$} & $<0.001$ \\
\hline & & & \multicolumn{4}{|c|}{ Bland-Altman } \\
\hline & & & \multicolumn{2}{|c|}{ Mean difference (ms) } & \multicolumn{2}{|c|}{ Limits of agreement (ms) } \\
\hline QTalg & vs. & QTobs1 & \multicolumn{2}{|l|}{3.39} & -23.23 & $: 30.01$ \\
\hline QTalg & vs. & QTobs2 & \multicolumn{2}{|l|}{-2.65} & -36.09 & $: 30.79$ \\
\hline QTalg & vs. & QTobs3 & \multicolumn{2}{|l|}{-1.88} & -31.53 & $: 27.78$ \\
\hline QTalg & vs. & HQTobs & \multicolumn{2}{|l|}{-0.38} & -25.41 & $: 24.65$ \\
\hline
\end{tabular}

Table 2-2 Inter-method variability 95\% Cl = 95\% confidence interval, obs = observer(s), r = Pearson's $r, \mathrm{p}=\mathrm{p}$-value, ICC $=$ intra-class coefficient, $\mathrm{ms}=$ milliseconds.

\subsubsection{Inter-observer variability}

Results of the comparison between observers are shown in Table 2-3. The agreement between all observers was strong (Pearson's $r$ ranging from 0.945 to 0.964 , ICC ranging from 0.939 to 0.958 ). The Bland-Altman analysis showed that the inter-observer bias ranged from $-0.74 \mathrm{~ms}$ to $6.28 \mathrm{~ms}$. The limits of agreement range from $26 \mathrm{~ms}$ to $31 \mathrm{~ms}$.

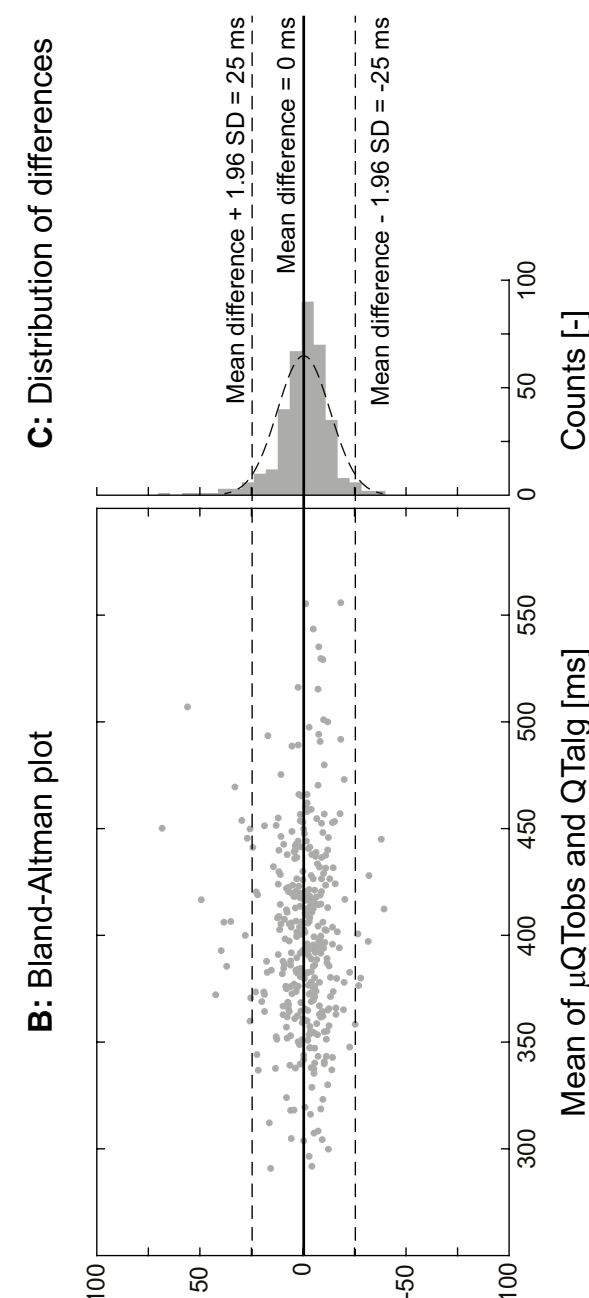

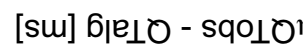

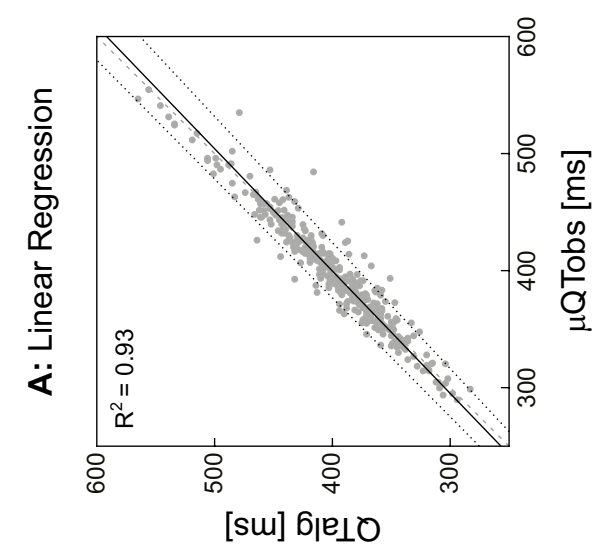




\begin{tabular}{|c|c|c|c|c|c|c|}
\hline \multicolumn{7}{|c|}{ Pearson correlation } \\
\hline & & & \multicolumn{3}{|c|}{$r(95 \% \mathrm{Cl})$} & $\mathrm{p}$ \\
\hline QTobs1 & vs. & QTobs2 & 0.947 & \multicolumn{2}{|c|}{$(0.935-0.957)$} & $<0.001$ \\
\hline QTobs1 & vs. & QTobs3 & 0.964 & \multicolumn{2}{|c|}{$(0.955-0.970)$} & $<0.001$ \\
\hline QTobs2 & vs. & QTobs3 & 0.945 & \multicolumn{2}{|c|}{$(0.934-0.956)$} & $<0.001$ \\
\hline & & & \multicolumn{4}{|c|}{ Intra-class coefficient } \\
\hline & & & \multicolumn{3}{|c|}{ ICC (95\% Cl) } & $\mathrm{p}$ \\
\hline QTobs1 & vs. & QTobs2 & 0.939 & \multicolumn{2}{|c|}{$(0.908-0.958)$} & $<0.001$ \\
\hline QTobs1 & vs. & QTobs3 & 0.958 & \multicolumn{2}{|c|}{$(0.932-0.972)$} & $<0.001$ \\
\hline \multirow[t]{3}{*}{ QTobs2 } & vs. & QTobs3 & 0.946 & \multicolumn{2}{|c|}{$(0.934-0.956)$} & $<0.001$ \\
\hline & & & \multicolumn{4}{|c|}{ Bland-Altman } \\
\hline & & & \multicolumn{2}{|c|}{ Mean difference (ms) } & \multicolumn{2}{|c|}{ Limits of agreement (ms) } \\
\hline QTobs1 & vs. & QTobs2 & \multicolumn{2}{|l|}{6.28} & \multicolumn{2}{|c|}{$-24.67: 37.23$} \\
\hline QTobs1 & vs. & QTobs3 & \multicolumn{2}{|l|}{5.54} & \multicolumn{2}{|c|}{$-20.41: 31.49$} \\
\hline QTobs2 & vs. & QTobs3 & \multicolumn{2}{|l|}{-0.74} & \multicolumn{2}{|c|}{$-31.06: 29.58$} \\
\hline
\end{tabular}

Table 2-3 Inter-observer variability 95\% Cl = 95\% confidence interval, obs = observer(s), $r=$ Pearson's $\mathrm{p}=\mathrm{p}$-value, $\mathrm{ICC}=$ intra-class coefficient, $\mathrm{ms}=$ milliseconds

\subsection{Discussion}

We have developed and validated an automatic QT-interval algorithm based on the tangent method which is unaffected by heart axis orientation and that can be applied on a beat-to-beat basis regardless of the T-wave morphology. There is a high agreement between the automatic algorithm and manual measurements of the QT-interval. Measuring errors between our algorithm and manual measurements are similar or even smaller than inter-observer measuring errors. In contrast to manual measurements, our algorithm enables users to study large amounts of complexes. Therefore, it can be used to study novel QT-interval parameters that require beat-to-beat QT-interval analysis.

\subsubsection{Measuring the QT-interval}

Recognition of an abnormal QT-interval is an important element to gain an impression of the risk for malignant arrhythmias and it guides treatment. However, determination of the QT-interval can be challenging ${ }^{4}$ and its result may frustrate treatment ${ }^{6}$. For manual QT assessment, the tangent method in lead II or V5 has been proposed. It has been suggested that with this method even inexperienced ECG readers can, after minimal education, accurately diagnose prolonged and normal QT-intervals $15,27$. However, manual assessment has considerable limitations. Proper manual QT-interval assessment is time consuming. Therefore, most physicians pick one lead and one complex to measure. Whether the QT-interval from the measured complex in the chosen lead is representative for the patient can be questionable. Measuring QT-intervals of multiple complexes over all leads is too time-consuming for daily clinical practice. Therefore, objective, standardized automated QT-interval algorithms unaffected by heart axis orientation are desirable.

\subsubsection{Algorithms by manufacturers}

All modern ECG machines provide users with automated measurements of ECG intervals. A genera downside of these algorithms is that the QT-interval is determined on an averaged complex over time (cf. Appendix Kligfield et al..13). Therefore, temporal fluctuations in QT-interval are lost and the dynamicity and adaptation of the QT-interval to changes in heart rate cannot be studied using these algorithms. Another downside of these algorithms is that the details about the algorithms are often unavailable for their users. Despite the latter, many cardiologists do use and trust the QTC provided by the ECG machine. Using a custom-made algorithm enables visualisation of the determined QRS-onset and T-wave end, making it easier to distinguish between correct and erroneous measurements.

\subsubsection{Custom-made QT-interval algorithms}

Custom-made (semi-)automated QT-interval algorithms were developed in order to study QT dynamics. Berger et al. ${ }^{14}$ for example, described a template matching algorithm to study QT dynamics. In his algorithm, a template (which is selected by the user) is matched to all complexes in order to measure individual QT-intervals. A disadvantage of his algorithm is that it only uses one ECG lead (I or II) and is therefore susceptible to heart axis orientation. For example, an algorithm that uses only lead II will most likely be unable to define $T_{\text {end }}$ in the ECG of the LQT2 patient as shown in Figure 2-3, due to the low T-wave amplitude in ECG lead II. Since our algorithm takes all leads into account, it is still able to define $\mathrm{T}_{\text {end }}$ as long as the T-wave is large enough in at least one lead (see Figure 2-3).

More sophisticated single- and multilead algorithms have also been reported22-26. However, a genera downside of those techniques is that they have not yet been validated for LQTS patients with various T-wave morphologies. Therefore it remains unknown how well these algorithms perform in T-wave morphologies alternated by LQTS. Almeida et al. proposed a multi-lead ECG delineation al gorithm which has been validated against multiple annotated database ${ }^{26}$. Almeida et al. report mean differences of $7.5 \pm 11.2 \mathrm{~ms}$ and $7.9 \pm 21.7 \mathrm{~ms}$ for the QRS-onset and T-wave end detection, respectively ${ }^{26}$. Although the exact mean differences in QT-interval can't be perceived from these results, the mean differences of QRS-onset and T-wave end detection suggest the differences in QT-interval to be similar to our validation results.

\subsubsection{Development}

Our algorithm is an extensive automated version of the tangent method first described by Lepeschkin and Surawicz. ${ }^{15}$ The tangent method has been shown to be an accurate and reproducible method for diagnosing prolonged QT-intervals, even by inexperienced ECG readers ${ }^{21}$. We applied this method to

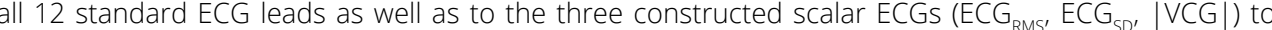
make it unaffected by heart axis orientation and applicable regardless of the T-wave morphology. Since prominent U-waves, notches, low T-waves and other altered T-wave morphologies often occur in only a few ECG leads, morphology-induced erroneous local $T_{\text {end }}$ detections will not affect the global $T_{\text {end. }}$.

It is important to bear in mind that though our algorithm is applicable regardless of the T-wave morphology, the T-wave morphology on its own can still be useful for the diagnosis of LQTS. 
The isoelectric baseline was defined as the median amplitude of the $30 \mathrm{~ms}$ preceding a QRS onset. The $\mathrm{P}-\mathrm{Q}$ segment was chosen instead of the $\mathrm{T}(\mathrm{U})-\mathrm{P}$ segment because the $\mathrm{PQ}$ segment is less affected by heart rate changes since at high heartrates the $\mathrm{P}$ wave can coincide with the T-wave.

\subsubsection{Validation}

The results of our validation study show good agreements between observers and our algorithm. The mean differences and limits of agreements between the observers and our algorithm are in the same range as the inter-observers' differences in this study as well as in a previous study ${ }^{27}$. The same holds for the results from ICC. This suggests that our algorithm is as accurate in determining the QT-interva as the observers.

The QT-intervals measured by the observers and the algorithm had an approximately norma distribution. To be sure not to make mistakes by using parametric tests, Spearman's correlation test and the Kendal's W coefficient of concordance were also computed and the results were compared with the Pearson's correlation test and the intra-class correlation coefficient. The differences between the parametric and non-parametric tests were small and the results of the non-parametric tests did not change the conclusion.

From our results we conclude that our algorithm is a good alternative for manual QT-interva measurements. Moreover, because the algorithm is unaffected by heart axis orientation and can provide beat-to-beat QT-intervals, it might have an additional value in diagnosing LQTS and evaluating new drugs.

\subsubsection{Advantages}

Additional advantages of our algorithm are that we are the first to combine T-wave landmarks derived from individual ECG leads with landmarks derived from scalar ECGs. By doing so, the algorithm combines the better of two worlds. The scalar ECGs are independent to heart axis orientation but since they are mean (ECG ${ }_{\text {nvs }}$, standard deviation (ECG ) or weighted mean (VCG) of individual ECG leads, information which is only present in one or two ECG leads is blurred and would have been lost if our algorithm wouldn't have used also the individual ECG leads. By calculating the median $T_{\text {end }}$ after outlier removal, the global $T_{\text {end }}$ is based on both the scalar ECGs and individual ECG leads. Secondly, the ECG ${ }_{\text {pus }}$ and ECG are calculated from unipolar ECG leads only. By doing so, all ECG leads contribute equally to the scalar ECGs. Another advantage is that our algorithm treats every complex individually and it does not require a priori knowledge. Methods like the one described by Ritsema van $\mathrm{Eck}^{23}$ might run into problems by sudden changes in T-wave morphology because each individual complex is cross-correlated with the average of the remainder complexes. Lastly, we described all necessary details to rebuild the algorithm and kept it as simple as possible. By doing so, the algorithm is reproducible and understandable for future users and clinicians.

\subsubsection{Limitations}

Although the algorithm had a high agreement with manual measurements, we acknowledge it has some limitations. First, low T-wave amplitude will result in a smaller signal-to-noise ratio and therefore might result in a larger error in $T_{\text {peak }}$ and $T_{\text {end }}$ detection. This is partially dealt with by excluding individua complexes on individual ECG leads if that particular complex has a T-wave amplitude smaller than 50 $\mathrm{\mu V}$. However, by excluding individual complexes on certain ECG leads, the remaining ECG leads become more important in those complexes. If respiration affects T-wave amplitude, a different number of individual ECG signals might be used for every complex within one respiratory cycle. This might induce detected QT variability.

Second, $T_{\text {end }}$ detection using the tangent approach is influenced by baseline deviations. Robust baseline determination techniques are rare and although the validation of our algorithm was successful, improving baseline determination might still improve the outcome.

Baumert et al. ${ }^{28}$ stated that conventional QT algorithms are not the best choice to measure beat-to-beat QT-interval changes. However, the conventional QT algorithm that has been studied in this article is a threshold method on the first derivative of a single lead ECG. Since our algorithm is based on the tangent method and takes all leads into account, the statement from Baumert et al..$^{28}$ can't be projected on our algorithm. To find out whether our algorithm can be used for beat-to-beat QT-interval parameters as described in the position paper from Baumert et al. ${ }^{9}$, a validation focused on these parameters is required.

astly, the observers measured the QT-interval from one lead only, while the algorithm takes all leads into account. However, since there is no true gold standard in the measurement of the QT-interval, we chose to validate the algorithm against the most objective manual assessment available.

\subsection{Conclusion}

Our validation results show that the QT-interval detection algorithm is as accurate in determining QTintervals as instructed manual observers are. Since the algorithm is fast, objective, unaffected by heart axis orientation, applicable regardless of the T-wave morphology and can provide beat-to-beat QTintervals, the algorithm might be useful to help improving the diagnosis of LQTS or the evaluation of QT-interval prolonging effects of new drugs. 


\subsection{References}

Moss, A. J. Long QT Syndrome. JAMA 289, 2041-2044 (2003).

2. Al-Khatib, S. M., LaPointe, N. M. A., Kramer, J. M. \& Califf, R. M. What Clinicians Should Know About the QT Interval. JAMA 289, 2120-2128 (2003).

3. International Conference on Harmonization of Technical for Registration of Pharmaceuticals for Human Use. The Clinical Evaluation of Qt/Qtc Interval Prolongation and Proarrhythmic Potential for Non-Antiarrhythmic Drugs: E14. (2005).

4. Viskin, S. et al. Inaccurate electrocardiographic interpretation of long QT: The majority of physicians cannot recognize a long QT when they see one. Heart Rhythm 2, 569-574 (2005)

5. Viskin, S. The QT interval: Too long, too short or just right. Heart Rhythm 6, 711-715 (2009).

6. Taggart, N. W., Haglund, C. M., Tester, D. J. \& Ackerman, M. J. Diagnostic miscues in congenital longQT syndrome. Circulation 115, 2613-2620 (2007).

7. Day, C. P., McComb, J. M. \& Campbell, R. W. QT dispersion: an indication of arrhythmia risk in patients with long QT intervals. Br. Heart J. 63, 342-344 (1990).

8. Franz, M. R. \& Zabel, M. Electrophysiological basis of QT dispersion measurements. Prog. Cardiovasc. Dis. 42, 311-324 (2000)

9. Baumert, M. et al. QT interval variability in body surface ECG: measurement, physiological basis, and clinical value: position statement and consensus guidance endorsed by the European Hear Rhythm Association jointly with the ESC Working Group on Cardiac Cellular Electroph. Europace 18 925-944 (2016)

10. Viskin, S. et al. The Response of the QT Interval to the Brief Tachycardia Provoked by Standing. J. Am Coll. Cardiol. 55, 1955-1961 (2010).

11. Adler, A. et al. The phenomenon of "QT stunning": The abnormal QT prolongation provoked by standing persists even as the heart rate returns to normal in patients with long QT syndrome. Heart Rhythm 9, 901-908 (2012)

12. Chorin, E. et al. Diagnostic value of T-wave morphology changes during "QT stretching" in patients with long QT syndrome. Heart Rhythm 12, 2263-2271 (2015).

3. Kligfield, P. et al. Comparison of automated measurements of electrocardiographic intervals and durations by computer-based algorithms of digital electrocardiographs. Am. Heart J. 167, 150-159. e1 (2014)

14. Berger, R. D. et al. Beat-to-Beat QT Interval Variability: Novel Evidence for Repolarization Lability in Ischemic and Nonischemic Dilated Cardiomyopathy. Circulation 96, 1557-1565 (1997).

15. Lepeschkin, E. \& Surawicz, B. The Measurement of the Q-T Interval of the Electrocardiogram. Circulation 6, 378-388 (1952).

16. Venkatachalam, K. L., Herbrandson, J. E. \& Asirvatham, S. J. Signals and signal processing for the electrophysiologist: Part I: Electrogram acquisition. Circ. Arrhythmia Electrophysiol. 4, 965-973 (2011).

17. Kors, J. A., van Herpen, G., Sittig, A. C. \& van Bemmel,J. H. Reconstruction of the Frankvectorcardiogram from standard electrocardiographic leads: diagnostic comparison of different methods. Eur. Heart J. 11, 1083-1092 (1990)

8. Pan, J. \& Tompkins, W. J. A real-time QRS detection algorithm. IEEE Trans. Biomed. Eng. 32, 230-6 (1985).

19. McGraw, K. O. \& Wong, S. P. Forming inferences about some intraclass correlations coefficients. Psychol. Methods 1, 30-46 (1996)
20. Bland, J. M. \& Altman, D. G. Comparing methods of measurement: why plotting difference against standard method is misleading. Lancet 346, 1085-1087 (1995).

21. Postema, P. G., De Jong J. S. S. G., Van der Bilt, I. A. C. \& Wilde, A. A. M. Accurate electrocardiographic assessment of the QT interval: Teach the tangent. Heart Rhythm 5, 1015-1018 (2008).

22. Starc, V. \& Schlegel, T. T. Real-time multichannel system for beat-to-beat QT interval variability. . Electrocardiol. 39, 358-367 (2006).

23. Ritsema van Eck, H. J. Fiducial segment averaging to improve cardiac time interval estimates. J. Electrocardiol. 35, 89-93 (2002)

24. Lux, R. L. et al. The application of root mean square electrocardiography (RMS ECG) for the detection of acquired and congenital long QT syndrome. PLoS One 9, e85689 (2014).

25. Schmidt, M. Baumert, M. Porta, A. Malbero H. \& Zaunseder, S. Two-Dimensional Warping for OneDimensional Signals-Conceptual Framework and Application to ECG Processing. IEEE Trans. Signa Process. 62, 5577-5588 (2014).

26. Almeida, R., Martinez, J. P., Rocha, A. P. \& Laguna, P. Multilead ECG Delineation Using Spatially Projected Leads From Wavelet Transform Loops. IEEE Trans. Biomed. Eng. 56, 1996-2005 (2009).

27. Panicker, G. K. et al. Intra- and interreader variability in QT interval measurement by tangent and threshold methods in a central electrocardiogram laboratory. J. Electrocardiol. 42, 348-352 (2009).

28. Baumert, M., Starc, V. \& Porta, A. Conventional QT variability measurement vs. template matching techniques: Comparison of performance using simulated and real ECG. PLoS One 7, (2012). 


\subsection{Supplementary Material}
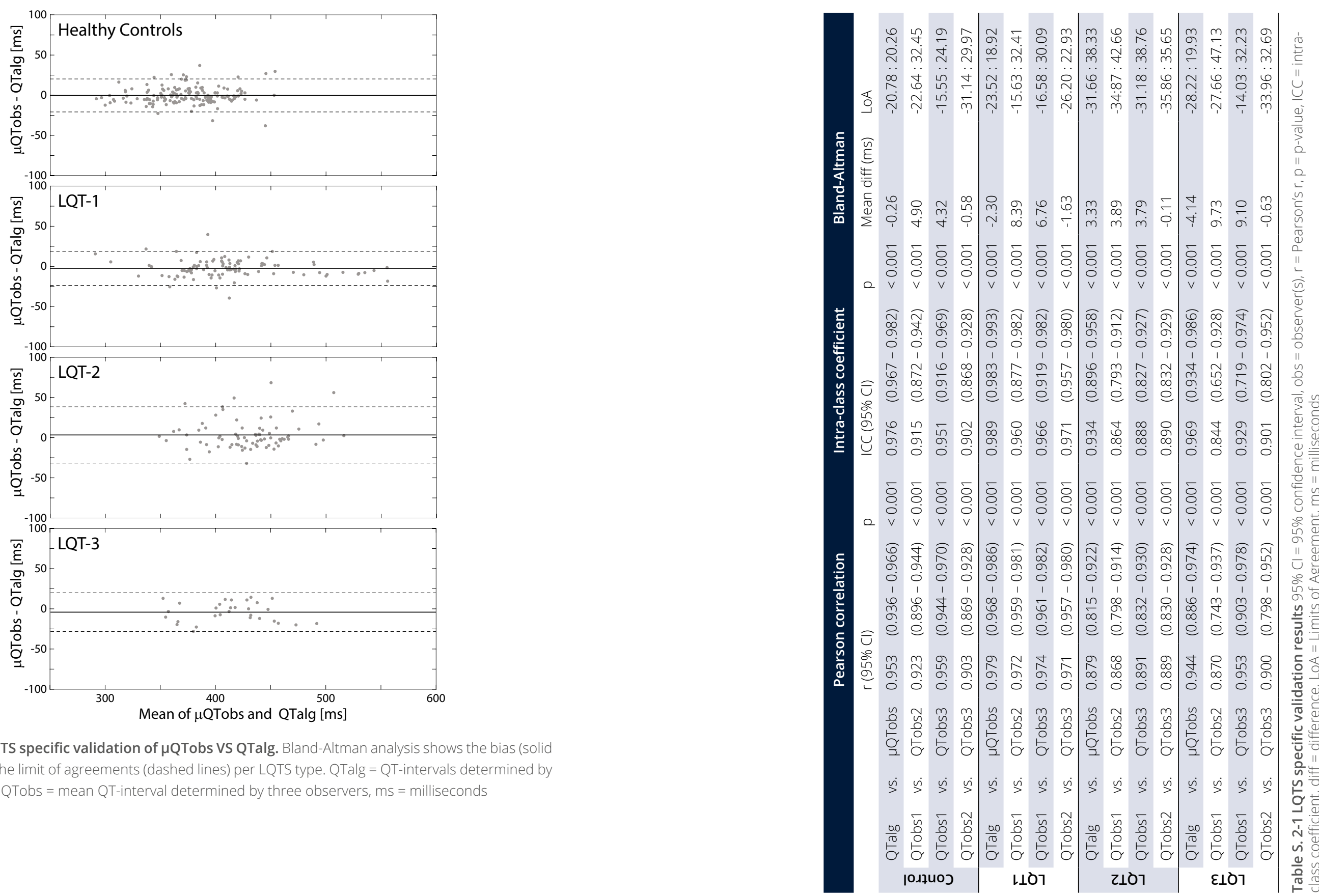

Figure S.2-1 LQTS specific validation of $\mu$ QTobs VS QTalg. Bland-Altman analysis shows the bias (solid black line) and the lmit of agreements (dashed lines) per LOTS type. QTalg = QT-Intervals determined by

the algorithm, $\mu \mathrm{QTobs}=$ mean QT-interval determined by three observers, $\mathrm{ms}=$ milliseconds 


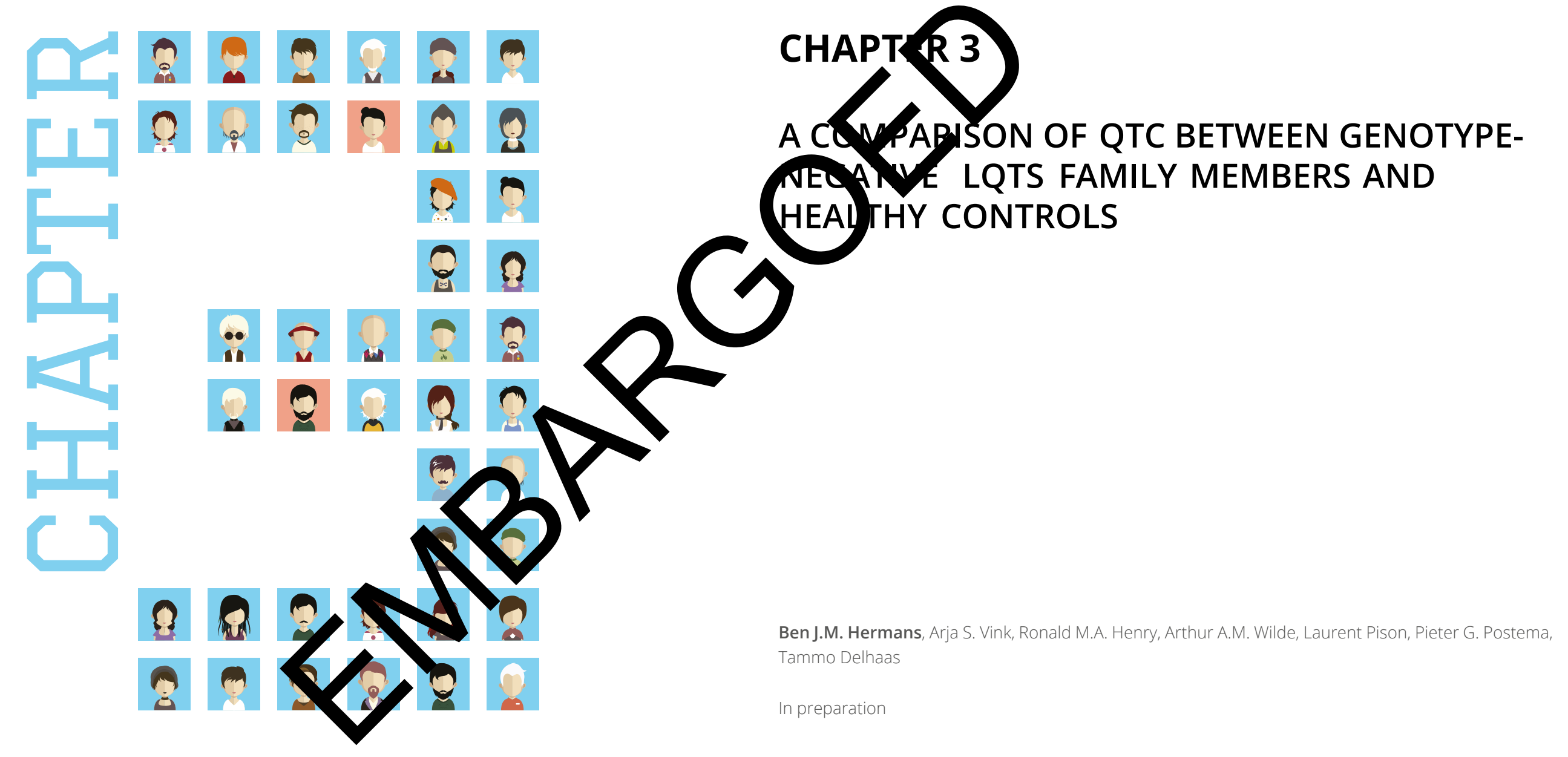




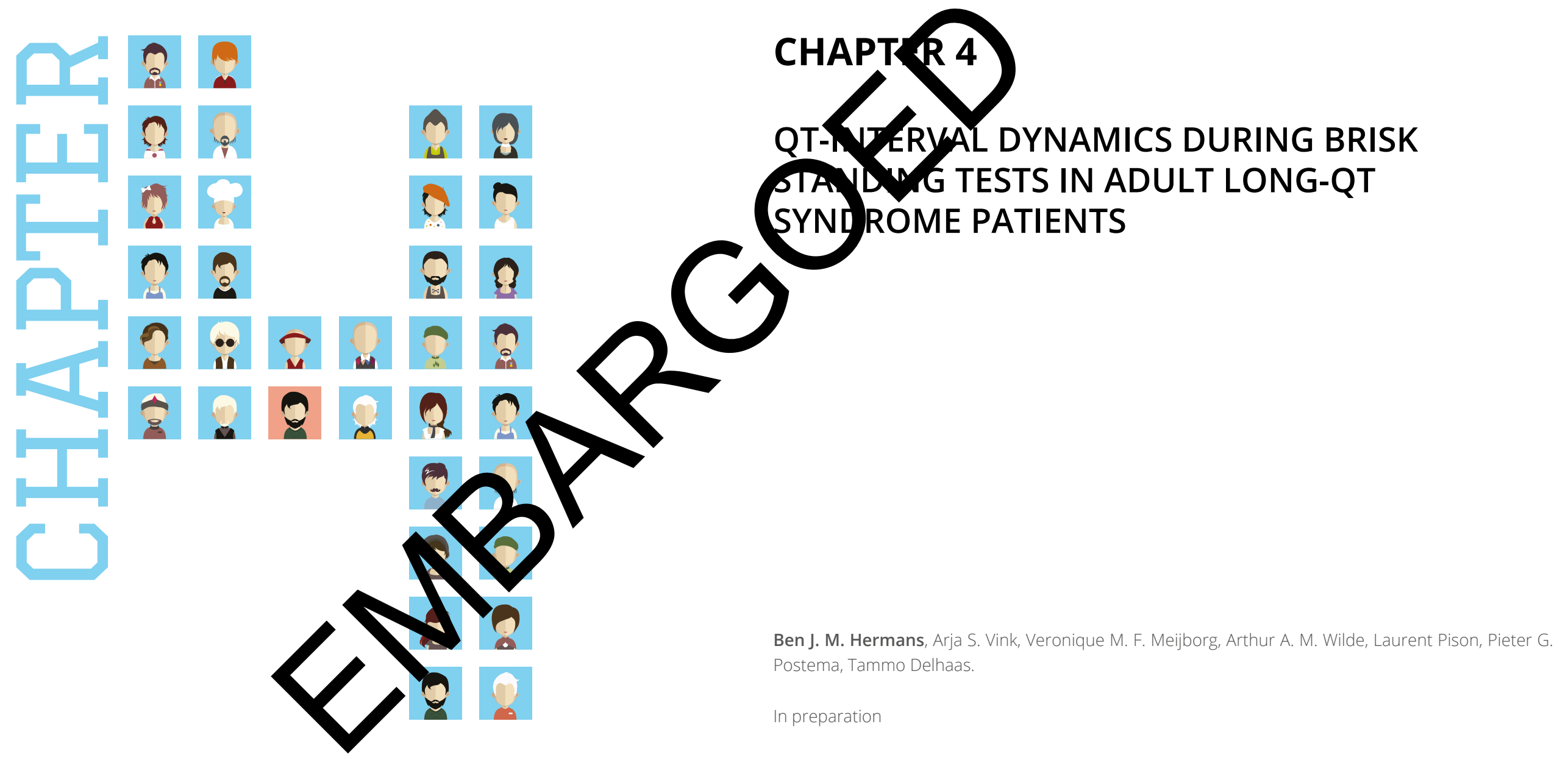




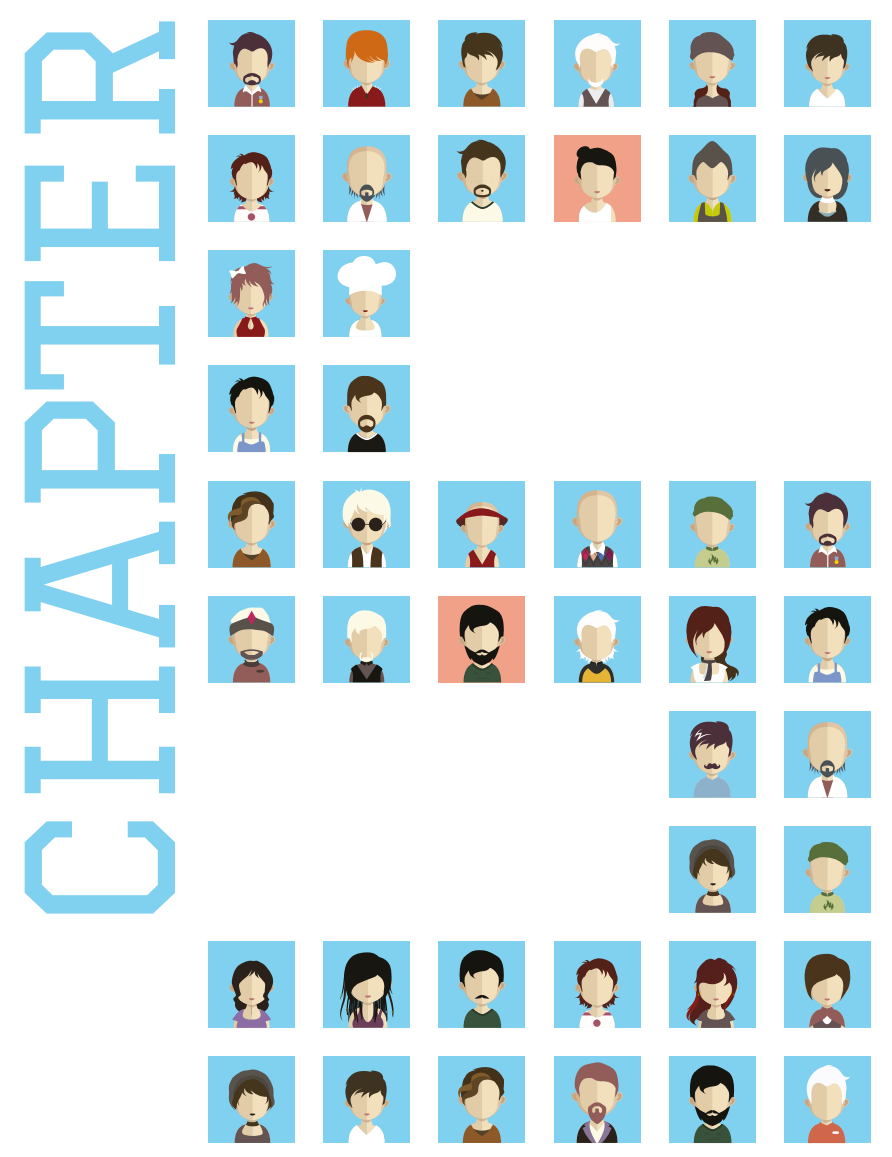

\section{CHAPTER 5}

DIAGNOSTIC ACCURACY OF THE QT-INTERVAL RESPONSE TO BRIEF TACHYCARDIA PROVOKED BY STANDING IN CHILDREN SUSPECT FOR LONG-QT SYNDROME 
Abstract

\section{Background:}

The brisk standing test has been shown to contain additional value in the diagnosis of the long-QT syndrome (LQTS) in adults. The diagnostic accuracy and cut-offs for a paediatric population remains unknown.

Objective

In this study we aim to evaluate the reproducibility of the standing test as well as its diagnostic value or the diagnosis of LQTS in children.

Methods

Electrocardiogram recordings of standing tests of genetically confirmed LQTS patients and LQTS genotype-negative controls were used in this study. The recordings were analysed manually in line with the initial studies on adults as well as automatically by a custom-made beat-to-beat QT-interva algorithm. The reproducibility was examined by inter- and intra-reader validity by Bland-Altman analyses and intra-class correlation coefficients. The diagnostic accuracy of the test was examined by receiver-operator curve $(\mathrm{ROC})$ analyses.

\section{Results}

A total of 87 controls and 47 LQTS patients were included for manual analyses. Digital ECGs were available for beat-to-beat analysis from 42 controls and 29 LQTS-patients. The inter- and intrareader validity of the measurements were good/high for all parameters. The diagnostic accuracy of the baseline QT-interval corrected for heart rate (QTc) resulted in the highest AUC (0.841). None of the "brisk standing test parameters" had a significant increased diagnostic value compared to the baseline QTC. Beat-to-beat analysis revealed that the QTC differed at between LQTS patients and controls at baseline and that this difference remained the same after standing.

\section{Conclusion}

QTC after standing did not differ between LQTS-children and controls. Therefore, the brisk standing test has no additional value as a screening test to diagnose LQTS as compared to a standard 10-second 12-leads resting ECG.

\subsection{Introduction}

Congenital long-QT syndrome (LQTS) is an inherited cardiac arrhythmia disorder associated with malignant ventricular arrhythmias especially in the young. The hallmark of the clinical diagnosis of LQTS is a prolongation of the QT-interval corrected for the heart rate (QTC) on a 12-lead rest electrocardiography (ECG). ${ }^{1,2}$ Besides a prolonged QTc, LQTS can also be diagnosed based on (I) the presence of clinical and other electrocardiographic features that are associated with LQTS or (II) the presence of a confirmed pathogenic genetic variant.1,2 These three elements in the diagnosis of LQTS are hampered by clinical challenges. LQTS-patients can have a borderline prolonged or even a normal resting QTC. ${ }^{3}$ Therefore a considerable overlap in QTc exists between affected and unaffected individuals. ${ }^{4}$ Furthermore, interpretation of symptoms as either benign or malignant is sometimes difficult5,6 whereas distinguishing pathogenic variants from innocuous rare variants can be very complex, especially in the current era of DNA-panels and whole-genome-sequencing. In addition, one third of the clinically diagnosed LQTSpatients have no mutation in one of the known pathogenic genes for LQTS.

Diagnosing LQTS remains therefore demanding. Consequently, additional tests to enhance diagnostic capacity such as QTc measurements during the recovery phase of exercise $e^{9,10}$ and during epinephrine infusion 11,12 have been developed. More recent, a "standing test" was developed, which showed that adult LQTS-patients with intermediate QTc at baseline, have an insufficient QT-interval shortening in response to the tachycardia provoked by abrupt standing compared to controls ${ }^{13}$ and that the QTC remained prolonged even after the heart rate returned to baseline. ${ }^{14}$

Children have a higher heart rate during resting conditions and a more pronounced reflex tachycardia compared to adults. ${ }^{15}$ When performing a "standing test" in healthy children, they have a QTc prolongation after standing that is more pronounced than in healthy adults. ${ }^{16}$ Therefore, using adult cut-off values for children may yield false positive results with the risk of incorrect LQTS-diagnosis and overtreatment. We aimed to further evaluate the diagnostic value of the "standing test" for the diagnosis of LQTS in children.

\subsection{Methods}

\subsubsection{Study design, setting and population}

We performed a prospective cohort study from January 2009 until September 2018. All patients under the age of 18 who underwent a "standing test" in the Amsterdam UMC, The Netherlands, were included. Patients received a "standing test" as part of regular care for (I) family screening in case of familial LQTS or sudden cardiac death (SCD) in the family, or (II) because of symptoms often in combination with a prolonged or high normal QTc on the 12-lead rest ECG.

The study was approved by the Academic Medical Center Review Board and informed consent of the individuals was waived as this study used retrospective data from regular care. 


\subsubsection{Data collection and measurements}

\section{ECGs and additional data}

From all patients, the first recorded standing test ECG was included. The "standing test" was performed

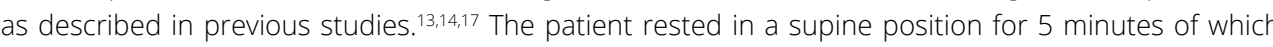
the final 2 minutes were recorded. Than they were asked to stand up quickly and remained standing for 3 minutes during continued ECG recording. Patient characteristics were collected including date of birth, sex, reason for genetic testing, and presence of cardiac events at baseline. At time of the ECGrecording the presence of beta-blocker therapy was also documented. Patients were stratified into four subgroups: (I) Controls (healthy individuals after cardiac screening due to a high normal/prolonged QTC and genotype-negative family members of LQTS patients), (II) LQTS (confirmed pathogenic variant in KCNQ1, KCNH2 and SCN5A), (III) Possible LQTS (Prolonged QTC and/or positive epinephrine test without a confirmed pathogenic variant, family members of genotype-negative LQTS-patients, suspected family history for LQTS), (IV) Other (screening for aborted cardiac arrest (ACA) or sudden cardiac death (SCD) in the family, idiopathic ventricular fibrillation (VF), cardiomyopathies, polymorphisms, Brugada Syndrome).

\section{Manual measurements}

All included standing-test ECGs were manually analyzed in line with previous studies.13.14 After standing, $<5$ seconds were precluded for measurements due to movement-related artefacts. One reader (SV) blinded for patient's characteristics measured the QT-interval and the preceding RR-interval at four points in time: (I) baseline, the first complex in supine position in which the RR-interval corresponds with the maximal sinus bradycardia after standing when the brief tachycardia is stabilized, (II) maximal tachycardia, during the maximal sinus rate as a response to standing, (III) maximal QT-interval stretching, defined at the time point after standing at which the end of the T-wave gets nearest to the next P-wave, (IV) return to baseline, during the maximal sinus bradycardia at standing position. In order to find matching RR-intervals in supine and standing position, first the complex with the longest preceding RRinterval within 30 seconds after standing was used for the measurements of return to baseline. Then the first complex during baseline with a corresponding RR-interval (RR-interval at return to baseline $+/$ - 40ms) was thereafter used for the baseline measurements.. This is a slight deviation of protoco compared to previous studies, ${ }^{13,14}$ to avoid exclusion of patients whose heart rate does not return to the RR-interval previously determined to be baseline.

At all stages the QT-interval was measured from the onset of the QRS-complex to the end of the T-wave using the tangent method and corrected for heart rate using Bazett's formula. All QT-intervals were measured in one lead preferably in lead II or V5.

\section{Validity of manual measurements}

To determine inter- and intra-reader validity for the measurements, a random sample of $10 \%$ was measured by an additional reader (IP) and re-measured by (SV).

\section{Automated measurements}

To study the dynamic response of the QT-interval to the abrupt change in heart rate in more detail, all digitally available standing-test ECGs were analysed beat-to-beat using custom-made software in MATLAB (2018a, Mathworks, Natick, MA, USA). After ECG filtering $(0.05-80 \mathrm{~Hz}$ bandpass filter $+50 \mathrm{~Hz}$ notch filter), R-peaks were detected using the Pan-Tompkins algorithm. ${ }^{18}$ QRS-onset and the end of the
T-wave were detected using a modified version of our previously described QT-interval algorithm..$^{19}$ In short, QRS-onset is detected as the first peak of the second derivative of the root mean square of the ECG preceding the R-peak. The end of the T-wave is detected using an automated tangent approach in which the interception of the baseline with a tangent through the last limb of the T-wave defines the end of the T-wave. Although the original algorithm used a median of the T-wave ends from all ECG leads as the global T-wave end, in this study we applied the algorithm to a single ECG lead to make the results more comparable with the manual QT-interval assessment. To compare the automated measurements to the manual measurements, single complexes where chosen based on the same definitions as the manual measurements: (I) baseline, (II) maximal tachycardia, (III) maximal QT-interval stretching and (IV) return to baseline. Furthermore, a moving average filter with a 15 second window with 5 second overlap was applied to the beat-to-beat QT- and RR-intervals for every subject. Then, the median (as well as the first and third quartiles) dynamic behaviours of these moving average intervals were calculated for controls and LQTS-patients. In addition, to further understand the QT-interval dynamics during standing we stratified the analyses for sex and LQTS-genotype (i.e. LQTS type 1 (LQT-1), LQTS type 2 (LQT-2) and LQTS type 3 (LQT-3).

\subsubsection{Statistical analysis}

All data were analyzed with R version 3.4.3. (The Foundation for Statistical Computing, Vienna, Austria). Baseline and ECG-characteristics were presented as numbers (percentage, \%) for categorical variables and mean ( \pm standard deviation, SD) or median (interquartiles) for continuous variables, stratified by group (i.e. control, LQTS, possible LQTS, other). Differences between the groups were tested using a $X^{2}$-test for categorical variables, and with a one-way ANOVA test or a Kruskal Wallis test for continuous variables as appropriate. For post-hoc analyses a Bonferroni correction was done in order to correct for multiple comparisons.

Inter- and intra-reader validity for manual measurements, and inter-method validity for the automatic measurements, were expressed as the intra-class correlation coefficient (ICC) for multiple measurements based on a two-way agreement (inter-reader validity) and consistency (intra-reader validity, inter-method validity) model according to $\mathrm{Cicchetti20}^{20}$ and Fleiss. ${ }^{21}$ Bland-Altman analyse ${ }^{22}$ were performed to assess bias and 95\% limits of agreement (LOA).

To test the diagnostic value of the standing test for LQTS, only controls and LQTS-patients were included and a receiver-operating characteristic $(R O C)$ curve analysis was used to calculate the area under the curve (AUC) and to evaluate the specificity at a predefined sensitivity of $90 \%$ (i.e. similar to earlier studies). ${ }^{13,14}$ DeLong's method ${ }^{23}$ was used to calculate the $95 \%$ confidence interval (CI) around the AUC and to compare ROC-curves.

A sensitivity analyses was performed by excluding all patients with an obviously long or obviously norma QTc at baseline because additional test can be considered superfluous for these individuals. Accordingly, we limited the analysis only including, controls and LQTS-patients with a baseline QTc under the 440.4

Sampling uncertainty was quantified with $95 \% \mathrm{Cl}$ and p-values. P-values $<0.05$ were considered to be statistically significant, but when corrected for multiple comparisons using the Bonferroni correction a $p$-value of $<0.002$ was considered to be statistically significant. 


\subsection{Results}

\subsubsection{Study population}

From a total of 180 individuals who were eligible for the study, 18 (10\%) had to be excluded because of inability to retrieve the standing-test ECGs and two (1\%) because standing-tests were insufficient due to a very low quality. The remaining 160 were included in the analysis. The study cohort consisted of 87 controls, 47 patients with LQTS (26 LQT-1, 19 LQT-2 and 2 LQT-3), 15 with possible LQTS, and 11 individuals with other final diagnoses (e.g. Brugada syndrome). Baseline characteristics of the included individuals are shown in Table 5-1. The four groups were of similar age, with a mean between 10 to 13 years old, but showed a difference in the number of included girls $(p=0.011)$. As expected, the groups differed in the reason for testing $(p<0.001)$, present symptoms at baseline $(p=0.002)$ and the number of patients that were on beta-blocker therapy $(p=0.002)$. Note that there were two controls on beta-blocker therapy, this was due to Wolff-Parkinson-White syndrome and due to hypertension.

\subsubsection{Manual measurements}

Table 5-1 shows the results for the manual measurements. As expected, the baseline QT-interval and QTc differed between the four groups ( $p<0.001$ for both) were LQTS-patients had the longest QT-interval and QTc compared to controls ( $p<0.001$ for both).

\section{Response to standing}

In standing position, the mean QT-interval and QTc differed per group during maximal tachycardia ( $p<0.001$ for both), at maximal QT-interval stretching $(p<0.001$ for both) and when the heart rate returned to baseline ( $\mathrm{p}<0.001$ for both). This was explained, by the longer QT-interval or QTc in LQTS-patients compared to controls ( $p<0.001$ for all comparisons). The mean heart rate after standing differed slightly between the groups.

As a response to standing, there was an increase in heart rate and the mean QT-interval at the point of maximal tachycardia and QT-interval stretching was shorter in all groups compared to baseline. Furthermore, QTc prolongation at maximal tachycardia and QT-interval stretching was present in all the groups, but did not differ between the groups ( $p=0.877$ and $p=0.594)$. The phenomenon of " $Q T$ stunning"14 (the persistence of QTc prolongation as the heart rate slows back to baseline) was present in especially LQTS-patients (delta QTC upon return to baseline heart rate 20, SD \pm 50 ) but did not significantly differ between the groups $(\mathrm{p}=0.059)$.

\section{Validity of manual measurements}

Supplementary Table S5-1, shows the inter- and intra-reader validity of the manual measurements. There was a good to high validity for all parameters, the QT-interval at return to baseline however, had the highest measurement variation (inter-reader 3ms, 95\% LoA $\pm 121 \mathrm{~ms}$; intra-reader 17ms, 95\% LoA $\pm 78 \mathrm{~ms}$ ).

\begin{tabular}{|c|c|c|c|c|c|}
\hline & $\begin{array}{l}\text { Control } \\
\mathrm{N}=87\end{array}$ & $\begin{array}{l}\text { LQTS } \\
\mathrm{N}=47\end{array}$ & $\begin{array}{l}\text { Pos. LQTS } \\
\mathrm{N}=15\end{array}$ & $\begin{array}{l}\text { Other } \\
\mathrm{N}=11\end{array}$ & $\mathrm{p}$ \\
\hline Age, years & $10(7-14)$ & $12(8-15)$ & $13(10-15)$ & $13(12-15)$ & 0.095 \\
\hline Girls & $39(45 \%)$ & $29(62 \%)$ & $11(73 \%)$ & $2(18 \%)$ & 0.011 \\
\hline Presentation & & & & & $<0.001$ \\
\hline Family screening & $48(55 \%)$ & $39(81 \%)$ & $3(20 \%)$ & $1(9 \%)$ & \\
\hline SCD in family & $8(9 \%)$ & $0(0 \%)$ & $1(7 \%)$ & $2(18 \%)$ & \\
\hline Near-drowning/OHCA/SCA & $1(1 \%)$ & $0(0 \%)$ & $2(13 \%)$ & $3(27 \%)$ & \\
\hline Other & $30(34 \%)$ & $9(19 \%)$ & $9(60 \%)$ & $5(45 \%)$ & \\
\hline Symptomatic at presentation & $1(1 \%)$ & $3(6 \%)$ & $2(13 \%)$ & $3(27 \%)$ & 0.002 \\
\hline On BB therapy & $2(2 \%)$ & $9(19 \%)$ & $4(27 \%)$ & $2(18 \%)$ & 0.002 \\
\hline \multicolumn{6}{|l|}{ Supine position¥ } \\
\hline$H R_{\text {baseine }}$ bpm & $80( \pm 15)$ & $73( \pm 16)$ & $74( \pm 17)$ & $71( \pm 13)$ & 0.032 \\
\hline $\mathrm{QT}_{\text {baseline }} \mathrm{ms}$ & $368( \pm 35)$ & $429( \pm 58)$ & $415( \pm 60)$ & $378( \pm 30)$ & $<0.001$ \\
\hline QT $c_{\text {baseline }}$ & $421( \pm 29)$ & $466( \pm 36)$ & $452( \pm 39)$ & $410( \pm 28)$ & $<0.001$ \\
\hline \multicolumn{6}{|l|}{ Standing position¥ } \\
\hline$H R_{\text {maxस }}, \mathrm{R}^{\prime} \mathrm{bpm}$ & $112( \pm 15)$ & $100( \pm 17)$ & $110( \pm 19)$ & $102( \pm 14)$ & 0.001 \\
\hline $\mathrm{QT}_{\text {maxtRR }} \mathrm{ms}$ & $361( \pm 35)$ & $421( \pm 61)$ & $393( \pm 56)$ & $374( \pm 44)$ & $<0.001$ \\
\hline QT $c_{\max H R}$ & $489( \pm 37)$ & $537( \pm 51)$ & $526( \pm 55)$ & $483( \pm 37)$ & $<0.001$ \\
\hline$H R_{\text {stretch' }}$ bpm & $110( \pm 16)$ & $99( \pm 17)$ & $109( \pm 20)$ & $99( \pm 11)$ & 0.002 \\
\hline $\mathrm{QT}_{\text {strecth }} \mathrm{ms}$ & $364( \pm 37)$ & $429( \pm 62)$ & $395( \pm 55)$ & $378( \pm 46)$ & $<0.001$ \\
\hline QT $c_{\text {stretch }}$ & $489( \pm 42)$ & $544( \pm 56)$ & $527( \pm 55)$ & $483( \pm 40)$ & $<0.001$ \\
\hline$H R_{\text {return' }}$ bpm & $81( \pm 14)$ & $74( \pm 16)$ & $78( \pm 16)$ & $71( \pm 11)$ & 0.024 \\
\hline $\mathrm{Q} T_{\text {return'ms }}$ & $372( \pm 39)$ & $450( \pm 74)$ & $405( \pm 61)$ & $376( \pm 26)$ & $<0.001$ \\
\hline QT $c_{\text {return }}$ & $429( \pm 37)$ & $492( \pm 60)$ & $456( \pm 70)$ & $406( \pm 35)$ & $<0.001$ \\
\hline \multicolumn{6}{|l|}{ Response to standing $¥$} \\
\hline Time to maximal tachycardia, $\mathrm{s}$ & $11(9-14)$ & $11(10-13)$ & $11(8-13)$ & $9(7-12)$ & 0.241 \\
\hline Time to maximal QT-stretching, s & $11(9-14)$ & $10(9-12)$ & $10(8-13)$ & $8(7-11)$ & 0.321 \\
\hline Time to return to baseline, $s$ & $22(18-27)$ & $20(19-29)$ & $20(17-29)$ & $22(18-24)$ & 0.904 \\
\hline$\triangle H R$ during maximal tachycardia,bpm & $32( \pm 11)$ & $27( \pm 9)$ & $36( \pm 9)$ & $30( \pm 11)$ & 0.010 \\
\hline$\Delta$ QT during maximal tachycardia,ms & $-10( \pm 22)$ & $-9( \pm 30)$ & $-22( \pm 35)$ & $-3( \pm 29)$ & 0.268 \\
\hline$\triangle$ QTc during maximal tachycardia & $66( \pm 41)$ & $71( \pm 47)$ & $73( \pm 47)$ & $75( \pm 49)$ & 0.877 \\
\hline$\Delta H R$ during maximal QT-stretching,bpm & $31( \pm 12)$ & $26( \pm 9)$ & $35( \pm 9)$ & $28( \pm 6)$ & 0.009 \\
\hline$\triangle$ QT during maximal QT-stretching,ms & $-7( \pm 21)$ & $0( \pm 39)$ & $-20( \pm 40)$ & $1( \pm 35)$ & 0.140 \\
\hline$\Delta$ QTc during maximal QT-stretching & $66( \pm 43)$ & $78( \pm 54)$ & $74( \pm 53)$ & $74( \pm 51)$ & 0.594 \\
\hline$\Delta \mathrm{HR}$ upon return to baseline $H R, b p m$ & $1( \pm 2)$ & $1( \pm 2)$ & $1( \pm 4)$ & $1( \pm 1)$ & 0.991 \\
\hline$\triangle Q T$ upon return to baseline $H R, m s$ & $5( \pm 29)$ & $22( \pm 47)$ & $-5( \pm 58)$ & $3( \pm 23)$ & 0.056 \\
\hline$\triangle Q T c$ upon return to baseline $H R$ & $7( \pm 32)$ & $26( \pm 50)$ & $-1( \pm 70)$ & $5( \pm 23)$ & 0.059 \\
\hline
\end{tabular}

Table 5-1. Baseline characteristics and manual ECG measurements. SCD=Sudden Cardiac Death, OHCA=Out of Hospital Cardiac Arrest, SCA=Sudden Cardiac Arrest, BB=beta-blocker, HR=heart rate, QTC= QT-interval corrected for heart rate using Bazett's formula, bpm= beats per minute, $\mathrm{ms}=$ milliseconds. $¥ \mathrm{p}$-values $<0.002$ are considered to be significant 
Diagnostic value

The QTC of LQTS-patients versus controls was already different at baseline, as shown in Table 5-1, and this difference remained present during standing with no difference in the response to standing between both groups (Supplementary Table S.5-2). As a consequence, the ROC curves demonstrate an AUC of 0.841 (95\% Cl 0.770-0.912) for baseline QTc but do not show a significant incremental diagnostic value for QTc during maximal tachycardia, during maximal QT-interval stretching or at return to baseline (Table 5-2). The baseline QTc that identified LQTS-patients with a 90\% sensitivity was 435 and yielded a $64 \%$ specificity.

\begin{tabular}{|c|c|c|c|c|}
\hline & AUIC & $95 \% \mathrm{Cl}$ & 90 & nsitivity \\
\hline chaseline & 0.841 & $0.770-0.912$ & 435 & $64 \%$ \\
\hline QT $c_{\operatorname{maxHR}}$ & 0.786 & $0.697-0.875$ & 476 & $40 \%$ \\
\hline QTc $c_{\text {stretch }}$ & 0.806 & $0.723-0.889$ & 491 & $63 \%$ \\
\hline QTC & 0.809 & $0.728-0.890$ & 422 & $44 \%$ \\
\hline
\end{tabular}

Table 5-2. Inter- and intra-reader validity. QTC= QT-interval corrected for heart rate using Bazett's formula, $\mathrm{AUC}=$ Area Under the Curve, $\mathrm{Cl}=$ confidence interval.

Sensitivity analyses manual measurements

ncluding only controls and LQTS-patients with a baseline QTc $<440 \mathrm{~ms}$, did not show any significan differences between the two groups (60 controls and 11 LQTS-patients) with respect to parameters during standing or response to standing (Supplementary Table S.5-3).

\subsubsection{Automated measurements}

A total of 84 individuals (53\%) had an available standing-test ECG, including 42 controls, 29 LQTSpatients, 10 possible-LQTS patients and 3 other individuals. Baseline characteristics, measurements at standing position and responds to standing did not show any major differences with the total cohort or with the direct manual measurements (Supplementary Table S.5-4 and Table S.5-5).

\section{Response to standing}

In order to study the dynamic response of the QT-interval to the abrupt change in heart rate in mor detail, we performed beat-to-beat analyses (Figure 5-1, 5-2 and 5-3). Figure 5-1 shows the data of the controls versus LQTS-patients. At baseline, LQTS-patients have a longer QT-interval and QTc with a lower hear rate compared to controls. This difference remains present after standing up (left column). After standing-up, LQTS-patients seem to get a higher heart rate about 30 seconds after standing compared to controls. While there is no difference in QTc adaptation, there is a decrease in absolute QT-interval in LQTS-patients as a consequence of the higher heart rate. This phenomenon seems to be more present in LOTS-boys (Figure 5-2, Supplementary Figure S.5-1) and in LQT-1 patients (Figure 5-3, Supplementary Figure S.5-2

\section{Validity of automated measurements}

Supplementary Table S.5-6, shows the inter-method validity between the automatic and the manua measurements. There was a good too high validity for almost all parameters, except for the QT-interval at return to baseline (ICC 0.774) with a systematic error of $20 \mathrm{~ms}$ (95\% LoA $\pm 84 \mathrm{~ms})$.
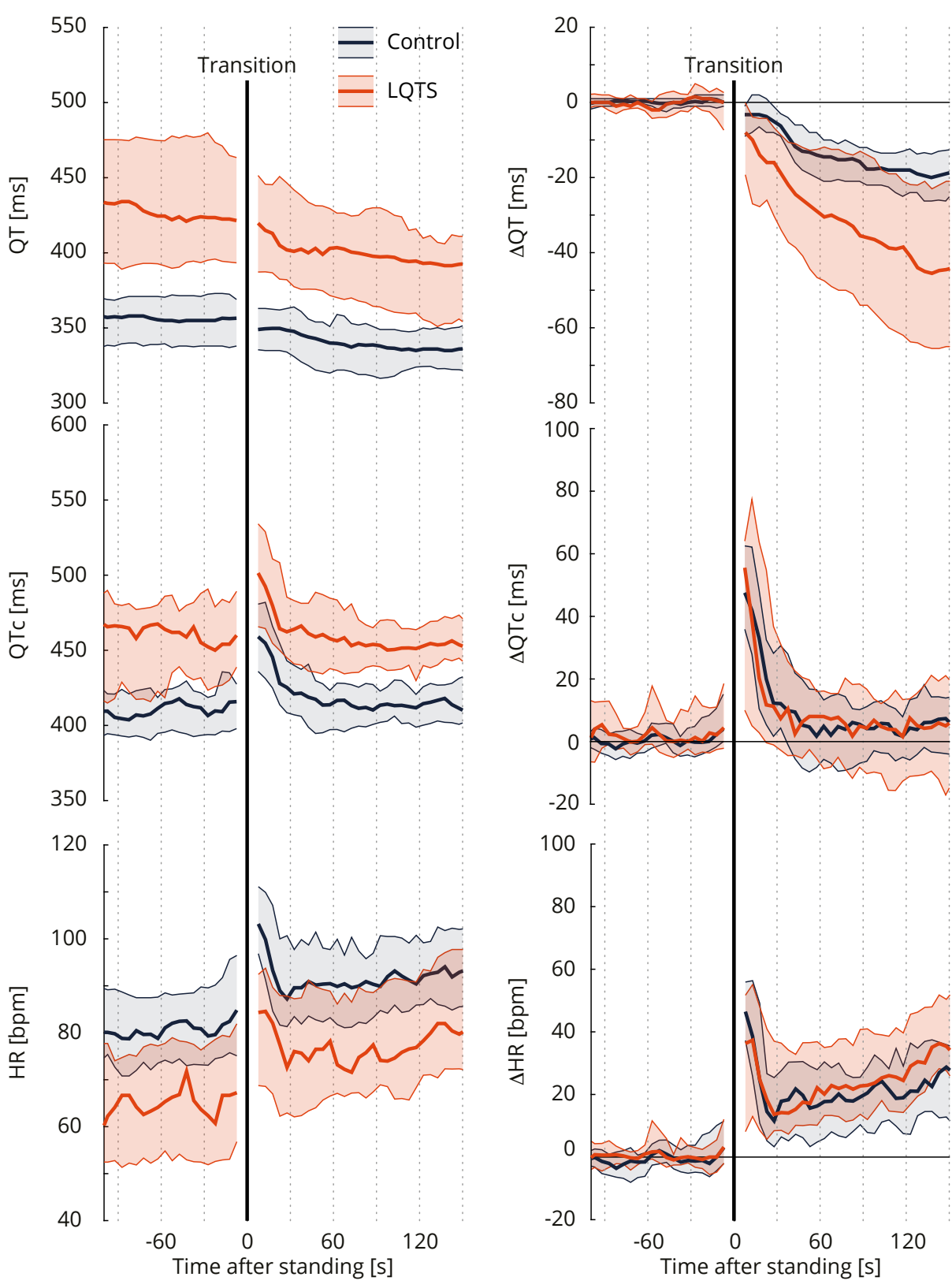

Figure 5-1 Dynamics of the standing test. Left column shows the median and interquartile range of the absolute QT-interval, QTc and heart rate of controls (blue) and LQTS-patients (orange). The right column shows the relative change of the QT-interval, QTC and heart rate to the baseline values for controls and LQTS-patients. Transition from supine to standing is indicated by the black solid line. HR= heart rate, LQTS= Long-QT syndrome, QTC= QT-interval corrected for heart rate using Bazett's formula. 

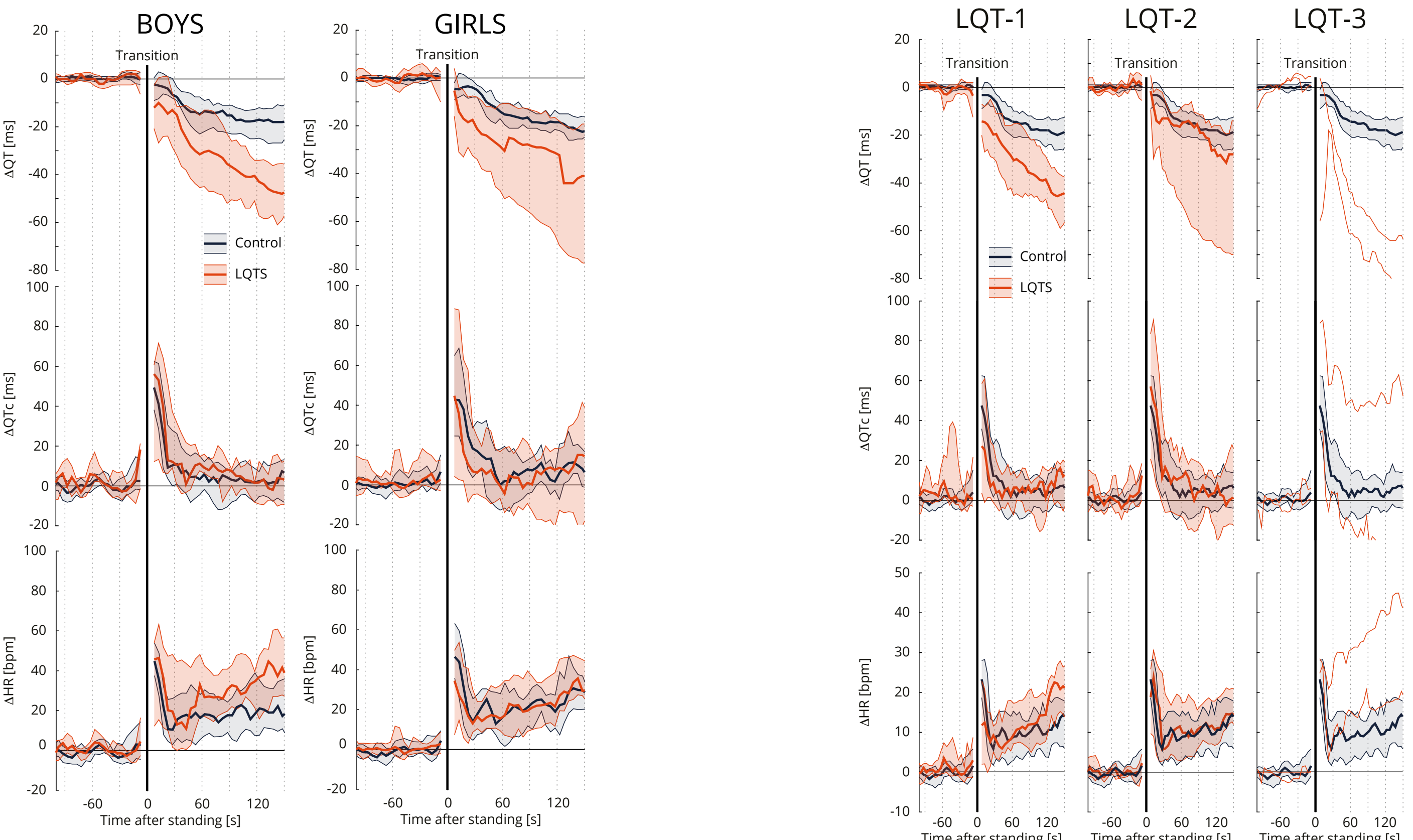

Figure 5-2 Sex difference in dynamics of the standing test. Including 36 boys ( 25 controls and 11 LQTS-patients) and 36 girls (18 controls and 18 LQTS-patients). The median and interquartile ranges of the relative change of the QT-interval, QTc and heart rate to the baseline for controls (blue) and LQTSpatients (orange), stratified for boys (left column) and girls (right column). Transition from supine to standing is indicated by the black solid line. HR= heart rate, LQTS= Long QT-syndrome, QTC= QT-interval corrected for heart rate using Bazett's formula.

Figure 5-3 Genotype differences in dynamics of the standing test. Including 14 LQT-1, 13 LQT-2 and 2 LQT-3 patients. Median and interquartile ranges of the relative change of the QT-interval, QTc and heart rate to the baseline for controls and LQTS type 1 (LQT-1), LQTS type 2 (LQT-2), LQTS type 3 (LQT3). Note that for LQT-3 we only showed the data of the 2 individual patients. Transition from supine to standing is indicated by the black solid line. HR= heart rate, LQTS= Long QT-syndrome, QTC= QT-interval corrected for heart rate using Bazett's formula. 


\subsection{Discussion}

An accurate diagnosis of LQTS is crucial as LQTS is a treatable but potentially lethal syndrome, especially in children. A LQTS diagnosis can however be difficult, with both under- and over diagnosis resulting in adverse events. ${ }^{24}$ Especially in children a correct diagnosis is of significant value because events may already occur in the very young and because there are many children who experience faints which could be due to vasovagal circumstances of e.g. due to malignant but self-terminating ventricular arrhythmias as can occur in LQTS. Because children often have pronounced sinus arrhythmia troubling QT-interval assessment, and because reference cohorts are often of a rather small size, test with additiona diagnostic value are appreciable. We here evaluated whether there could be an added diagnostic value of the standing test in children and we found that the test had no clear additional value as a screening test to diagnose LQTS and to discriminate (borderline) LQTS-patients from healthy children as compared to QTc obtained from a standard resting ECG

\subsubsection{Diagnostic value}

The normal response to standing is a sudden acceleration of the heart rate with a gradual shortening of the QT-interval. Since the adaptation of the QT-interval to sudden changes in heart rate is delayed ('QThysteresis'), the vagally mediated reflex tachycardia after standing results in a transiently prolongation of the QTC. This phenomenon is present in healthy individuals but in adults, LQTS-patients entirely fail to shorten the QT-interval during the short-lasting tachycardia provoked by standing (impaired QThysteresis) resulting in a longer QTc compared to healthy individuals. ${ }^{13,14}$ In healthy children, however, it was shown that the QT-interval barely decreased after standing and that QTC subsequently prolonged mainly due to heart rate changes induced by standing ${ }^{16,25}$ The more important role of the heart rate in children compared to adults was expected, since age has a negative correlation with postural heart rate increments. ${ }^{15}$ This was reflected in the decreased time to maximal heart rate and greater magnitude of heart rate change in healthy children compared to healthy adults as shown in previous studies. ${ }^{13,16}$

In our cohort, the controls showed a slight QT-interval shortening $(-10 \mathrm{~ms}, \pm 22 \mathrm{~ms})$ after standing compared to previous findings (Oms) in children, ${ }^{16,25}$ but considerable less compared to healthy adults $(-21 \mathrm{~ms}, \pm 19 \mathrm{~ms}){ }^{13}$ The observed QTc prolongation $(66 \mathrm{~ms}, \pm 41)$ was subsequently mainly dependent on the heart rate change induced by standing. In LQTS-children, there was an approximately equal degree in QT-interval shortening, QTc prolongation and heart rate change compared to healthy children. Hence, the standing test did not add diagnostic value upon the baseline QT (AUC 0.786 for QTcmaxHR versus AUC 0.841 for QTcbase). Excluding individuals with an obvious long QTc, however, show a trend towards a QT-interval prolongation after standing in LQTS-children, which was previous seen in LQTS-adults.13

The sudden heart rate acceleration produced by standing not only increases the QTc but it has been described that it also exposes abnormal T waves that are valuable for diagnosing LQTS.1

\subsubsection{Dynamic response to standing}

As QT-hysteresis involves the direct and short-term QT-interval adaptation to sudden heart rate changes, it relates to dynamic changes. With the evaluation of selected single time points, as performed in the standing test, dynamic responses can be analyzed suboptimal. We performed beat-to-beat, non-steady- state analyses, in order to evaluate the QT- interval and heart rate responds to standing in LQTS-patients and controls, In particular, the heart rate and heart rate responds to standing seemed to be of an important value.

At baseline, LQTS-patients had a lower heart rate compared to controls. One could argue that this is due to a difference in beta-blocker therapy (controls 2\% versus 19\% in LQTS-patients), however the absolute number of patients on beta-blocker therapy is very low. It is known that LQTS- fetuses have a subtle slower fetal heart rate $(<3 \text { rd percentile for gestational age })^{26,27}$. Hence, gene-carriers can have a lower heart rate compared to controls, in addition to the effect of beta-blocker therapy in our study.

In the normal responds to standing, once in the upright position, the blood volume shifts from the thorax into the legs, pelvis and abdomen due to gravitational pooling. Therefore, there is less blood available for the heart to eject (i.e. decreased venous return and preload). As a consequence, there is a decrease in cardiac filling, stroke volume and cardiac output resulting in a steep fall in blood pressure ${ }^{28}$ which a causes the removal of the tonic inhibition of the baro-mechanoreceptors (baroreflex), inducing a decrease in parasympathic (vagal) activity and a consequent increase in heart rate within 3 seconds. A more gradual secondary increase in heart rate, starting around 5 seconds after stand up, is mainly due to further reflex inhibition of cardiac vagal tone and increased sympathetic outflow to the sinus node and can be attributed to diminished activation of arterial baroreceptors by the fall in arteria pressure. ${ }^{29}$ In LQTS-patients, cardiac events are often elicited by an increased sympathetic activity (i.e. swimming/diving or sudden loud noise) $)^{30}$ and the initiation of these arrhythmias can be suppressed by beta-adrenergic blocking agents ${ }^{31}$ or by ablation of the left sympathetic chain ganglion. ${ }^{32} \mathrm{It}$ is therefore thought that LQTS-patients have an imbalance of the autonomic nervous system, with a more prominent sympathetic tonus. It is however unclear whether the autonomic balance at rest or the dynamicity in autonomic activity is more relevant. In this study we noticed that LQTS-patients had a slightly more gradual increase in heart rate after standing up compared to controls, which can be the result of a more prominent sympathetic tonus. In addition, it is interesting to see that this phenomenon is more present in boys and LQT-1 patients as it is known that LQT-1 patients have their cardiac events during increased sympathetic activity (i.e. swimming) and that LQT-1 males have a higher risk during childhood and an earlier onset of cardiac events than LQT-1 females. ${ }^{33-35}$

\subsubsection{Limitations}

This study encounters several limitations. First, as the study is part of regular care we are hampered with problems of 'real-world data'. Some patients had to start beta-blocker therapy before the performance of the standing test because of comorbidity (e.g. hypertension, supraventricular tachycardia) or due to a cardiac event during exercise without a clear cause. Although the absolute number of patients on beta-blocker therapy is low, it could be a confounding factor. Second, the number of included patients was limited and although a larger number was naturally wanted, the originally study of Viskin et al.1 included a similar cohort size (68 LQTS-patients and 82 controls) but did show significant differences between LQTS-patients and controls. Third, standing tests were not performed by the same investigator at a standard time of the day, nor where we informed about the pre-test physical activity. This could all have had effect on the heart rate and repolarization. ${ }^{36}$ Furthermore, we have no data on the intrasubject variability of the test as we did nog repeated the test in the same subject which hampers us to say something about reproducibility. 


\subsection{Conclusion}

The QTc after standing is more pronounced in children compared to adults as a consequence of a higher heart rate responds, but did not differ between LQTS-children and controls. Compared to differences in baseline QTc between LQTS-children and controls, we could not determine additional diagnostic value of the standing test as a screening test to diagnose LQTS in children. However, analyzing beat-to-beat heart rate and QT-interval dynamics during the standing test may have a role in the risk-stratification of LQTS-children.

\subsection{Acknowledgments}

We thank Veronique M.F. Meijborg, Christian van der Werf, Louise R. Olde Nordkamp and Sebastién P. Krul, for their invaluable help in collecting ECGs for this study.

\subsection{References}

1. Priori, S. G. et al. 2015 ESC Guidelines for the management of patients with ventricular arrhythmias and the prevention of sudden cardiac death the Task Force for the Management of Patients with Ventricular Arrhythmias and the Prevention of Sudden Cardiac Death of the Europea. Eur. Heart J. 36, 2793-2867l (2015)

2. Priori, S. G. et al. HRS/EHRA/APHRS Expert Consensus Statement on the Diagnosis and Management of Patients with Inherited Primary Arrhythmia Syndromes: Document endorsed by HRS, EHRA, and APHRS in May 2013 and by ACCF, AHA, PACES, and AEPC in June 2013. Heart Rhythm 10, 1932 1963 (2013).

3. Vincent, G. M., Timothy, K. W., Leppert, M. \& Keating, M. The Spectrum of Symptoms and QT Intervals in Carriers of the Gene for the Long-QT Syndrome. N. Engl. . . Med. 327, 846-852 (1992).

4. Vink, A. S. et al. Determination and Interpretation of the QT Interval. Circulation 138, 2345-2358 (2018).

5. Moya, A. et al. Guidelines for the diagnosis and management of syncope (version 2009). Eur. Heart J. 30, 2631-2671 (2009)

6. van Dijk, N. et al. High diagnostic yield and accuracy of history, physical examination, and ECG in patients with transient loss of consciousness in FAST: the Fainting Assessment study. J. Cardiovasc. Electrophysiol. 19, 48-55 (2008).

7. Wilde, A. A. M. \& Ackerman, M. J. Exercise extreme caution when calling rare genetic variants novel arrhythmia syndrome susceptibility mutations. Heart Rhythm 7, 1883-1885 (2010).

8. Giudicessi, J. R. \& Ackerman, M. J. Genotype- and Phenotype-Guided Management of Congenita Long QT Syndrome. Curr. Probl. Cardiol. 38, 417-455 (2013).

9. Sy, R. W. et al. Derivation and validation of a simple exercise-based algorithm for prediction of genetic testing in relatives of LQTS probands. Circulation 124, 2187-2194 (2011).

10. Horner, J. M., Horner, M. M. \& Ackerman, M. J. The diagnostic utility of recovery phase QTC during treadmill exercise stress testing in the evaluation of long QT syndrome. Heart Rhythm 8, 1698 1704 (2011).

11. Vyas, H., Hejlik, J. \& Ackerman, M. J. Epinephrine QT Stress Testing in the Evaluation of Congenital Long-QT Syndrome. Circulation 113, 1385-1392 (2006).

12. Shimizu, W. et al. Diagnostic value of epinephrine test for genotyping LQT1, LQT2, and LQT3 forms of congenital long QT syndrome. Heart Rhythm 1, 276-283 (2004).

3. Viskin, S. et al. The Response of the QT Interval to the Brief Tachycardia Provoked by Standing. J. Am. Coll. Cardiol. 55, 1955-1961 (2010).

14. Adler, A. et al. The phenomenon of "QT stunning": The abnormal QT prolongation provoked by standing persists even as the heart rate returns to normal in patients with long QT syndrome. Heart Rhythm 9, 901-908 (2012).

15. Ives, C. T. \& Kimpinski, K. Higher postural heart rate increments on head-up tilt correlate with younger age but not orthostatic symptoms. J. Appl. Physiol. 115, 525-528 (2013).

16. Filippini, L. H. P. M. et al. The brisk standing-test for long QT syndrome in prepubertal schoo children: defining normal. EP Eur. 20, f108-f112 (2018).

17. Chorin, E. et al. Diagnostic value of T-wave morphology changes during "QT stretching" in patients with long QT syndrome. Heart Rhythm 12, 2263-2271 (2015).

18. Pan, J. \& Tompkins, W. J. A real-time QRS detection algorithm. IEEE Trans. Biomed. Eng. 32, 230-6 (1985) 
19. Hermans, B. J. M. et al. The development and validation of an easy to use automatic QT-interval algorithm. PLoS One 12, e0184352 (2017)

20. Cicchetti, D. V \& Sparrow, S. A. Developing criteria for establishing interrater reliability of specific items: applications to assessment of adaptive behavior. Am. J. Ment. Defic. 86, 127-37 (1981).

21. Fleiss, J., Levin, B. \& Cho Paik, M. Statistical Methods for Rates and Proportions. John Wiley \& Sons (2003). doi:10.1198/tech.2004.5812

22. Bland, J. M. \& Altman, D. G. Comparing methods of measurement: why plotting difference against standard method is misleading. Lancet 346, 1085-1087 (1995).

23. DeLong, E. R., DeLong, D. M. \& Clarke-Pearson, D. L. Comparing the Areas under Two or More Correlated Receiver Operating Characteristic Curves: A Nonparametric Approach. Biometrics 44, 837 (1988).

24. Taggart NW, Haglund CM, Tester DJ and Ackerman MJ. Diagnostic miscues in congenital long-QT syndrome. Circulation. 2007;115:2613-20

25. Dionne, A. et al. Dynamic QT Interval Changes from Supine to Standing in Healthy Children. Can. J. Cardiol. 34, 66-72 (2018)

26. Mitchell, J. L. et al. Fetal heart rate predictors of long QT syndrome. Circulation 126, 2688-2695 (2012)

27. Winbo, A. et al. Third Trimester Fetal Heart Rate Predicts Phenotype and Mutation Burden in the Type 1 Long QT Syndrome. Circ. Arrhythmia Electrophysiol. 8, 806-814 (2015).

28. Borst, C., Van Brederode, J. F. M. Wieling W. Van Montfrans, G. A. \& Dunning A. J. Mechanisms of Initial Blood Pressure Response to Postural Change. Clin. Sci. 67, 321-327 (1984).

29. Wieling, W. \& Karemaker, J. M. Measurement of heart rate and blood pressure to evaluate disturbances in neurocardiovascular control. in Autonomic Failure 290-306 (Oxford University Press, 2013). doi:10.1093/med/9780198566342.003.0025

30. Moss, A. J. et al. The long QT syndrome. Prospective longitudinal study of 328 families. Circulation 84, 1136-1144 (1991)

31. Priori, S. G. et al. Association of long QT syndrome loci and cardiac events among patients treated with beta-blockers. JAMA 292, 1341-4 (2004).

32. Schwartz, P. J. et al. Left Cardiac Sympathetic Denervation in the Management of High-Risk Patients Affected by the Long-QT Syndrome. Circulation 109, 1826-1833 (2004).

33. Costa, J. et al. Combined assessment of sex-and mutation-specific information for risk stratification in type 1 long QT syndrome. Heart Rhythm 9, 892-898 (2012).

34. Locati, E. H. et al. Age- and Sex-Related Differences in Clinical Manifestations in Patients With Congenital Long-QT Syndrome. Circulation 97, 2237-2244 (1998).

35. Zareba, W. et al. Modulating effects of age and gender on the clinical course of long QT syndrome by genotype. J. Am. Coll. Cardiol. 42, 103-109 (2003).

36. Porta, A. et al. Autonomic Control of Heart Rate and QT Interval Variability Influences Arrhythmic Risk in Long QT Syndrome Type 1. J. Am. Coll. Cardiol. 65, 367-374 (2015).

\subsection{Supplementary materials}

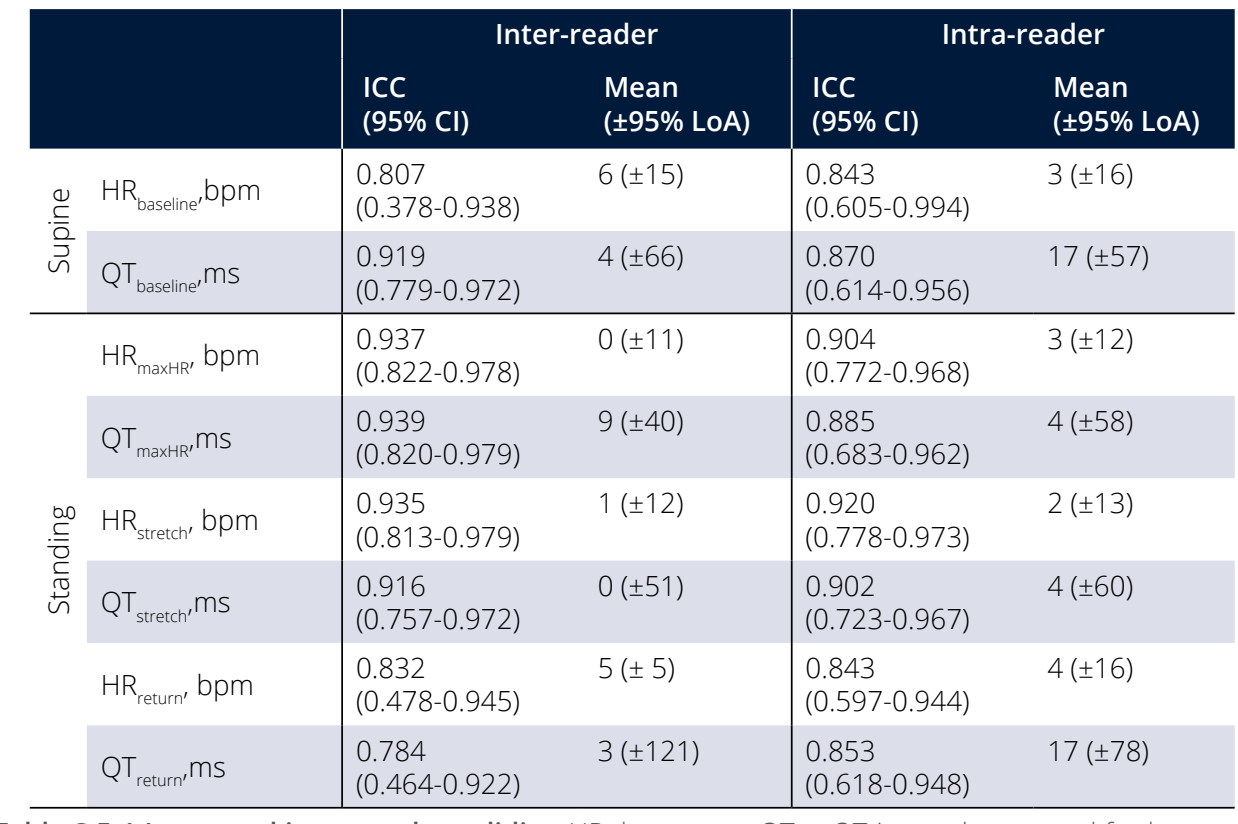

Table S.5-1 Inter- and intra-reader validity. HR=heart rate, QTC= QT-interval corrected for heart rate using Bazett's formula, bpm= beats per minute, ms= milliseconds, ICC= intraclass correlation coefficient, $\mathrm{IC}=$ confidence interval, LoA= Limits of agreement. 


\begin{tabular}{|c|c|c|c|}
\hline & Control $\mathrm{N}=87$ & LQTS N=47 & $\mathrm{p}$ \\
\hline Age, years & $10(7-14)$ & $12(8-15)$ & 1.000 \\
\hline Girls & $39(45 \%)$ & $29(62 \%)$ & 0.554 \\
\hline Presentation & & & $<0.001$ \\
\hline Family screening & $48(55 \%)$ & $39(81 \%)$ & \\
\hline SCD in family & $8(9 \%)$ & $0(0 \%)$ & \\
\hline Near-drowning/OHCA/SCA & $1(1 \%)$ & $0(0 \%)$ & \\
\hline Other & $30(34 \%)$ & $9(19 \%)$ & \\
\hline Symptomatic at presentation & $1(1 \%)$ & $3(6 \%)$ & 1.000 \\
\hline On BB therapy & $2(2 \%)$ & $9(19 \%)$ & 0.010 \\
\hline \multicolumn{4}{|l|}{ Supine position $¥$} \\
\hline$H R_{\text {baseline }}, \mathrm{bpm}$ & $80( \pm 15)$ & $73( \pm 16)$ & 0.069 \\
\hline $\mathrm{QT}_{\text {baseline' }} \mathrm{ms}$ & $368( \pm 35)$ & $429( \pm 58)$ & $<0.001$ \\
\hline QTC $c_{\text {baseline }}$ & $421( \pm 29)$ & $466( \pm 36)$ & $<0.001$ \\
\hline \multicolumn{4}{|l|}{ Standing position $¥$} \\
\hline$H R_{\text {maxtR' }}$ bpm & $112( \pm 15)$ & $100( \pm 17)$ & 0.001 \\
\hline $\mathrm{QT}_{\text {maxHRR }} \mathrm{ms}$ & $361( \pm 35)$ & $421( \pm 61)$ & $<0.001$ \\
\hline QT $c_{\max H R}$ & $489( \pm 37)$ & $537( \pm 51)$ & $<0.001$ \\
\hline$H R_{\text {strectch }}$ bpm & $110( \pm 16)$ & $99( \pm 17)$ & 0.002 \\
\hline $\mathrm{QT}_{\text {stretch }} \mathrm{ms}$ & $364( \pm 37)$ & $429( \pm 62)$ & $<0.001$ \\
\hline QT $c_{\text {stretch }}$ & $489( \pm 42)$ & $544( \pm 56)$ & $<0.001$ \\
\hline$H R_{\text {return' }} \mathrm{bpm}$ & $81( \pm 14)$ & $74( \pm 16)$ & 0.047 \\
\hline $\mathrm{QT} T_{\text {return }} \mathrm{ms}$ & $372( \pm 39)$ & $450( \pm 74)$ & $<0.001$ \\
\hline QT $c_{\text {return }}$ & $429( \pm 37)$ & $492( \pm 60)$ & $<0.001$ \\
\hline \multicolumn{4}{|l|}{ Response to standing $¥$} \\
\hline Time to maximal tachycardia, s & $11(9-14)$ & $11(10-13)$ & 1.000 \\
\hline Time to maximal QT-stretching, s & $11(9-14)$ & $10(9-12)$ & 1.000 \\
\hline Time to return to baseline, $\mathrm{s}$ & $22(18-27)$ & $20(19-29)$ & 1.000 \\
\hline$\Delta H R$ during maximal tachycardia,bpm & $32( \pm 11)$ & $27( \pm 9)$ & 0.050 \\
\hline$\Delta \mathrm{QT}$ during maximal tachycardia,ms & $-10( \pm 22)$ & $-9( \pm 30)$ & 1.000 \\
\hline$\triangle$ QTc during maximal tachycardia & $66( \pm 41)$ & $71( \pm 47)$ & 1.000 \\
\hline$\Delta H R$ during maximal QT-stretching,bpm & $31( \pm 12)$ & $26( \pm 9)$ & 0.070 \\
\hline$\triangle \mathrm{QT}$ during maximal QT-stretching,ms & $-7( \pm 21)$ & $0( \pm 39)$ & 1.000 \\
\hline$\triangle$ QTc during maximal QT-stretching & $66( \pm 43)$ & $78( \pm 54)$ & 1.000 \\
\hline$\Delta H R$ upon return to baseline $H R, b p m$ & $1( \pm 2)$ & $1( \pm 2)$ & 1.000 \\
\hline$\triangle \mathrm{QT}$ upon return to baseline $\mathrm{HR}, \mathrm{ms}$ & $5( \pm 29)$ & $22( \pm 47)$ & 0.120 \\
\hline$\triangle Q T c$ upon return to baseline $H R$ & $7( \pm 32)$ & $26( \pm 50)$ & 0.095 \\
\hline
\end{tabular}

Table 5.5-2. Baseline characteristics and manual ECG measurements for post-hoc analyses between controls and LQTS-patients. SCD=Sudden Cardiac Death, OHCA=Out of Hospital Cardiac Arrest, SCA=Sudden Cardiac Arrest, BB=beta-blocker, HR=heart rate, $\mathrm{QTC}=\mathrm{QT}$-interval corrected for heart rate using Bazett's formula, bpm= beats per minute, ms= milliseconds. $¥ p$-values $<0.002$ are considered to be significant

\begin{tabular}{|c|c|c|c|}
\hline & Control $\mathrm{N}=60$ & LQTS N=11 & $\mathrm{p}$ \\
\hline Age, years & $10(7-13)$ & $8(8-15)$ & 0.768 \\
\hline Girls & $26(43 \%)$ & $7(64 \%)$ & 0.362 \\
\hline Presentation & & & 0.606 \\
\hline Family screening & $38(63 \%)$ & $9(82 \%)$ & \\
\hline SCD in family & $5(8 \%)$ & $0(0 \%)$ & \\
\hline Near-drowning/OHCA/SCA & $1(2 \%)$ & $0(0 \%)$ & \\
\hline Other & $16(27 \%)$ & $2(18 \%)$ & \\
\hline Symptomatic at presentation & $1(2 \%)$ & $0(0 \%)$ & 1.000 \\
\hline On BB therapy & $0(0 \%)$ & $1(9 \%)$ & 0.155 \\
\hline \multicolumn{4}{|l|}{ Supine position¥ } \\
\hline$H R_{\text {baseline }}$ bpm & $78( \pm 14)$ & $76( \pm 13)$ & 0.621 \\
\hline $\mathrm{QT}_{\text {baseline }} \mathrm{ms}$ & $360( \pm 34)$ & $380( \pm 28)$ & 0.054 \\
\hline QT $c_{\text {baseline }}$ & $406( \pm 19)$ & $424( \pm 23)$ & 0.027 \\
\hline \multicolumn{4}{|l|}{ Standing position $¥$} \\
\hline$H R_{\text {maxHR' }}$ bpm & $112( \pm 16)$ & $107( \pm 9)$ & 0.162 \\
\hline $\mathrm{QT}_{\operatorname{maxkR}} \mathrm{ms}$ & $355( \pm 33)$ & $381( \pm 43)$ & 0.076 \\
\hline QT $c_{\text {maxHR }}$ & $481( \pm 31)$ & $507( \pm 56)$ & 0.149 \\
\hline$H R_{\text {strecth }^{\prime}}$ bpm & $110( \pm 16)$ & $104( \pm 9)$ & 0.136 \\
\hline $\mathrm{QT}_{\text {strecthr }}, \mathrm{ms}$ & $357( \pm 35)$ & $393( \pm 50)$ & 0.042 \\
\hline $\mathrm{QT} c_{\text {strecth }}$ & $479( \pm 39)$ & $516( \pm 62)$ & 0.079 \\
\hline$H R_{\text {return, }}$ bpm & $79( \pm 14)$ & $80( \pm 11)$ & 0.767 \\
\hline $\mathrm{QT}_{\text {reurrm }} \mathrm{ms}$ & $367( \pm 37)$ & $399( \pm 47)$ & 0.066 \\
\hline QT $c_{\text {return }}$ & $416( \pm 30)$ & $458( \pm 52)$ & 0.032 \\
\hline \multicolumn{4}{|l|}{ Response to standing $¥$} \\
\hline$\Delta H R$ during maximal tachycardia,bpm & $35( \pm 10)$ & $31( \pm 8)$ & 0.153 \\
\hline$\Delta \mathrm{QT}$ during maximal tachycardia,ms & $-9( \pm 18)$ & $1( \pm 38)$ & 0.405 \\
\hline$\Delta$ QTc during maximal tachycardia & $74( \pm 34)$ & $83( \pm 56)$ & 0.606 \\
\hline$\Delta H R$ during maximal QT-stretching,bpm & $34( \pm 11)$ & $28( \pm 7)$ & 0.048 \\
\hline$\Delta \mathrm{QT}$ during maximal QT-stretching,ms & $-9( \pm 21)$ & $13( \pm 44)$ & 0.140 \\
\hline$\Delta$ QTc during maximal QT-stretching & $73( \pm 40)$ & $93( \pm 62)$ & 0.324 \\
\hline$\Delta \mathrm{HR}$ upon return to baseline $\mathrm{HR}, \mathrm{bpm}$ & $0( \pm 2)$ & $1( \pm 2)$ & 0.179 \\
\hline$\Delta \mathrm{QT}$ upon return to baseline $H R, \mathrm{~ms}$ & $8( \pm 25)$ & $21( \pm 45)$ & 0.388 \\
\hline$\triangle Q T c$ upon return to baseline $H R$ & $10( \pm 27)$ & $13( \pm 56)$ & 0.325 \\
\hline
\end{tabular}

Table S.5-3. Baseline characteristics and manual ECG measurements of the sensitivity analyses including only QTC $<440$ (95th percentile) on baseline ECG. SCD=Sudden Cardiac Death, OHCA=Out of Hospital Cardiac Arrest, SCA=Sudden Cardiac Arrest, BB=beta-blocker, HR=heart rate, QTC= QTinterval corrected for heart rate using Bazett's formula, bpm= beats per minute, $\mathrm{ms}=$ milliseconds. p-values $<0.002$ are considered to be significant 


\begin{tabular}{|c|c|c|c|c|c|}
\hline & $\begin{array}{l}\text { Control } \\
\mathrm{N}=42\end{array}$ & $\begin{array}{l}\text { LQTS } \\
N=29\end{array}$ & $\begin{array}{l}\text { Pos. LQTS } \\
\mathrm{N}=10\end{array}$ & $\begin{array}{l}\text { Other } \\
\mathrm{N}=3\end{array}$ & $\mathrm{p}$ \\
\hline Age, years & $9(6-13)$ & $13(10-15)$ & $14(11-17)$ & $16(14-16)$ & 0.015 \\
\hline Girls & $18(43 \%)$ & $18(62 \%)$ & $6(60 \%)$ & $1(33 \%)$ & 0.314 \\
\hline Presentation & & & & & $<0.001$ \\
\hline Family screening & $18(43 \%)$ & $25(86 \%)$ & $2(20 \%)$ & $0(0 \%)$ & \\
\hline SCD in family & $1(2 \%)$ & $0(0 \%)$ & $0(0 \%)$ & $0(0 \%)$ & \\
\hline Near-drowning/OHCA/SCA & $1(2 \%)$ & $0(0 \%)$ & $2(20 \%)$ & $1(33 \%)$ & \\
\hline Other & $22(52 \%)$ & $4(14 \%)$ & $6(60 \%)$ & $2(67 \%)$ & \\
\hline Symptomatic at presentation & $1(2 \%)$ & $2(9 \%)$ & $2(20 \%)$ & $1(33 \%)$ & 0.046 \\
\hline On BB therapy & $1(2 \%)$ & $6(21 \%)$ & $3(30 \%)$ & $0(0 \%)$ & 0.014 \\
\hline \multicolumn{6}{|l|}{ Supine position $¥$} \\
\hline $\mathrm{HR}_{\text {baseline }} \mathrm{t} \mathrm{bm}$ & $78( \pm 12)$ & $69( \pm 16)$ & $72( \pm 20)$ & $59( \pm 6)$ & 0.030 \\
\hline $\mathrm{QT}_{\text {baseline }} \mathrm{ms}$ & $356( \pm 27)$ & $433( \pm 56)$ & $381( \pm 57)$ & $375( \pm 30)$ & $<0.001$ \\
\hline QT $c_{\text {baseline }}$ & $403( \pm 28)$ & $455( \pm 36)$ & $407( \pm 27)$ & $373( \pm 49)$ & $<0.001$ \\
\hline \multicolumn{6}{|l|}{ Standing position $¥$} \\
\hline$H R_{\max H R^{\prime}} \mathrm{bpm}$ & $115( \pm 13)$ & $99( \pm 15)$ & $111( \pm 23)$ & $91( \pm 4)$ & $<0.001$ \\
\hline $\mathrm{QT}_{\text {maxHR }}$ ms & $347( \pm 32)$ & $421( \pm 56)$ & $281( \pm 51)$ & $373( \pm 28)$ & $<0.001$ \\
\hline QT $c_{\max +R}$ & $479( \pm 34)$ & $536( \pm 45)$ & $509( \pm 50)$ & $460( \pm 28)$ & $<0.001$ \\
\hline$H R_{\text {strecth}^{\prime}}$ bpm & $113( \pm 14)$ & $97( \pm 16)$ & $109( \pm 23)$ & $90( \pm 3)$ & $<0.001$ \\
\hline $\mathrm{QT}_{\text {stretch }}$ 'ms & $355( \pm 31)$ & $430( \pm 60)$ & $395( \pm 51)$ & $372( \pm 30)$ & $<0.001$ \\
\hline QT $c_{\text {stretch }}$ & $486( \pm 35)$ & $539( \pm 44)$ & $526( \pm 54)$ & $456( \pm 32)$ & $<0.001$ \\
\hline $\mathrm{HR}_{\text {return, }} \mathrm{bpm}$ & $78( \pm 14)$ & $68( \pm 14)$ & $75( \pm 21)$ & $60( \pm 6)$ & 0.018 \\
\hline$Q T_{\text {reurnm }}$ ms & $359( \pm 33)$ & $432( \pm 49)$ & $390( \pm 49)$ & $371( \pm 30)$ & $<0.001$ \\
\hline QT $c_{\text {return }}$ & $406( \pm 44)$ & $454( \pm 43)$ & $426( \pm 41)$ & $372( \pm 47)$ & $<0.001$ \\
\hline \multicolumn{6}{|l|}{ Response to standing $¥$} \\
\hline Time to maximal tachycardia, s & $11(10-14)$ & $12(11-15)$ & $11(8-14)$ & $8(8-10)$ & 0.445 \\
\hline Time to maximal QT-stretching, s & $11(9-13)$ & $10(9-13)$ & $11(9-13)$ & $7(7-10)$ & 0.692 \\
\hline Time to return to baseline, $\mathrm{s}$ & $26(22-28)$ & $24(21-28)$ & $25(23-27)$ & $20(17-23)$ & 0.624 \\
\hline$\triangle H R$ during maximal tachycardia,bpm & $38( \pm 12)$ & $31( \pm 9)$ & $38( \pm 14)$ & $32( \pm 8)$ & 0.045 \\
\hline$\Delta \mathrm{QT}$ during maximal tachycardia,ms & $-9( \pm 23)$ & $-12( \pm 26)$ & $0( \pm 22)$ & $-2( \pm 10)$ & 0.568 \\
\hline$\triangle$ QTc during maximal tachycardia & $77( \pm 36)$ & $81( \pm 39)$ & $102( \pm 51)$ & $86( \pm 24)$ & 0.316 \\
\hline$\Delta H R$ during maximal QT-stretching,bpm & $36( \pm 12)$ & $28( \pm 9)$ & $37( \pm 14)$ & $31( \pm 7)$ & 0.034 \\
\hline$\Delta \mathrm{QT}$ during maximal QT-stretching,ms & $-1( \pm 17)$ & $-3( \pm 26)$ & $14( \pm 27)$ & $-3( \pm 8)$ & 0.183 \\
\hline$\Delta$ QTc during maximal QT-stretching & $84( \pm 33)$ & $85( \pm 40)$ & $118( \pm 51)$ & $82( \pm 19)$ & 0.066 \\
\hline$\triangle H R$ upon return to baseline $H R, b p m$ & $0( \pm 8)$ & $-1( \pm 10)$ & $3( \pm 8)$ & $0( \pm 2)$ & 0.594 \\
\hline$\Delta Q T$ upon return to baseline $H R, m s$ & $4( \pm 17)$ & $0( \pm 25)$ & $9( \pm 22)$ & $-2( \pm 6)$ & 0.599 \\
\hline$\triangle Q T C$ upon return to baseline $H R$ & $4( \pm 30)$ & $-2( \pm 34)$ & $22( \pm 31)$ & $-2( \pm 12)$ & 0.247 \\
\hline
\end{tabular}

\begin{tabular}{|c|c|c|c|c|c|}
\hline & $\begin{array}{l}\text { Control } \\
\mathrm{N}=42\end{array}$ & $\begin{array}{l}\text { LQTS } \\
N=29\end{array}$ & $\begin{array}{l}\text { Pos. LQTS } \\
\mathrm{N}=10\end{array}$ & $\begin{array}{l}\text { Other } \\
\mathrm{N}=3\end{array}$ & $\mathrm{p}$ \\
\hline \multicolumn{6}{|l|}{ Supine position¥ } \\
\hline$H R_{\text {baseline }} \mathrm{bpm}$ & $82( \pm 11)$ & $70( \pm 16)$ & $73( \pm 20)$ & $61( \pm 7)$ & 0.001 \\
\hline $\mathrm{QT}_{\text {baseline }}, \mathrm{ms}$ & $362( \pm 27)$ & $443( \pm 57)$ & $417( \pm 73)$ & $362( \pm 27)$ & $<0.001$ \\
\hline QT $c_{\text {baseline }}$ & $423( \pm 26)$ & $469( \pm 37)$ & $448( \pm 43)$ & $405( \pm 43)$ & $<0.001$ \\
\hline \multicolumn{6}{|l|}{ Standing position $¥$} \\
\hline$H R_{\max H R^{\prime}} \mathrm{bpm}$ & $116( \pm 14)$ & $98( \pm 17)$ & $110( \pm 24)$ & $91( \pm 3)$ & $<0.001$ \\
\hline $\mathrm{QT}_{\text {maxसR }} \mathrm{ms}$ & $355( \pm 35)$ & $431( \pm 59)$ & $388( \pm 65)$ & $393( \pm 50)$ & $<0.001$ \\
\hline QTC $c_{\text {maxtr }}$ & $491( \pm 42)$ & $544( \pm 48)$ & $517( \pm 61)$ & $484( \pm 55)$ & $<0.001$ \\
\hline$H R_{\text {stretch, }}, \mathrm{bpm}$ & $115( \pm 14)$ & $97( \pm 18)$ & $120( \pm 24)$ & $91( \pm 30)$ & $<0.001$ \\
\hline $\mathrm{QT}_{\text {strecth }}, \mathrm{ms}$ & $357( \pm 37)$ & $442( \pm 58)$ & $384( \pm 63)$ & $393( \pm 50)$ & $<0.001$ \\
\hline QT $c_{\text {stretch }}$ & $490( \pm 43)$ & $554( \pm 54)$ & $510( \pm 53)$ & $484( \pm 55)$ & $<0.001$ \\
\hline$H R_{\text {return, }}$ bpm & $83( \pm 12)$ & $69( \pm 14)$ & $79( \pm 20)$ & $62( \pm 9)$ & $<0.001$ \\
\hline $\mathrm{QT}_{\text {returrm }} \mathrm{ms}$ & $367( \pm 37)$ & $475( \pm 72)$ & $398( \pm 69)$ & $360( \pm 0)$ & $<0.001$ \\
\hline $\mathrm{QT} c_{\text {retum }}$ & $429( \pm 37)$ & $505( \pm 62)$ & $449( \pm 79)$ & $365( \pm 26)$ & $<0.001$ \\
\hline \multicolumn{6}{|l|}{ Response to standing $¥$} \\
\hline Time to maximal tachycardia, s & $10(8-13)$ & $10(9-12)$ & $10(7-13)$ & $7(7-10)$ & 0.674 \\
\hline Time to maximal QT-stretching, s & $10(8-12)$ & $10(9-12)$ & $10(7-12)$ & $7(7-10)$ & 0.698 \\
\hline Time to return to baseline, $\mathrm{s}$ & $20(17-23)$ & $20(17-22)$ & $19(17-21)$ & $20(18-21)$ & 0.963 \\
\hline$\Delta H R$ during maximal tachycardia,bpm & $33( \pm 11)$ & $28( \pm 10)$ & $37( \pm 10)$ & $31( \pm 6)$ & 0.066 \\
\hline$\Delta \mathrm{QT}$ during maximal tachycardia,ms & $-7( \pm 23)$ & $-11( \pm 30)$ & $-29( \pm 41)$ & $-10( \pm 36)$ & 0.189 \\
\hline$\triangle$ QTc during maximal tachycardia & $69( \pm 38)$ & $75( \pm 50)$ & $69( \pm 56)$ & $78( \pm 60)$ & 0.937 \\
\hline$\Delta H R$ during maximal QT-stretching,bpm & $32( \pm 11)$ & $27( \pm 10)$ & $37( \pm 10)$ & $31( \pm 6)$ & 0.066 \\
\hline$\Delta \mathrm{QT}$ during maximal QT-stretching,ms & $-4( \pm 20)$ & $-1( \pm 42)$ & $-33( \pm 41)$ & $-10( \pm 36)$ & 0.056 \\
\hline$\triangle$ QTc during maximal QT-stretching & $69( \pm 38)$ & $85( \pm 57)$ & $61( \pm 57)$ & $79( \pm 60)$ & 0.449 \\
\hline$\Delta H R$ upon return to baseline $H R, b p m$ & $1( \pm 2)$ & $0( \pm 2)$ & $1( \pm 5)$ & $1( \pm 2)$ & 0.654 \\
\hline$\triangle Q T$ upon return to baseline $H R, m s$ & $5( \pm 27)$ & $32( \pm 55)$ & $-11( \pm 63)$ & $-25( \pm 7)$ & 0.018 \\
\hline$\Delta \mathrm{QTc}$ upon return to baseline $\mathrm{HR}$ & $7( \pm 29)$ & $36( \pm 56)$ & $-6( \pm 77)$ & $-20( \pm 10)$ & 0.034 \\
\hline
\end{tabular}

Table S.5-5. Manual measurements of the automatic measured ECGs. HR=heart rate, QTc= QTinterval corrected for heart rate using Bazett's formula, bpm= beats per minute, ms= milliseconds. $¥$ p-values $<0.002$ are considered to be significant

Table S.5-4. Baseline characteristics and automatic ECG measurements. HR=heart rate, QTC= QTinterval corrected for heart rate using Bazett's formula, bpm= beats per minute, ms=milliseconds. $p$-values $<0.002$ are considered to be significant 

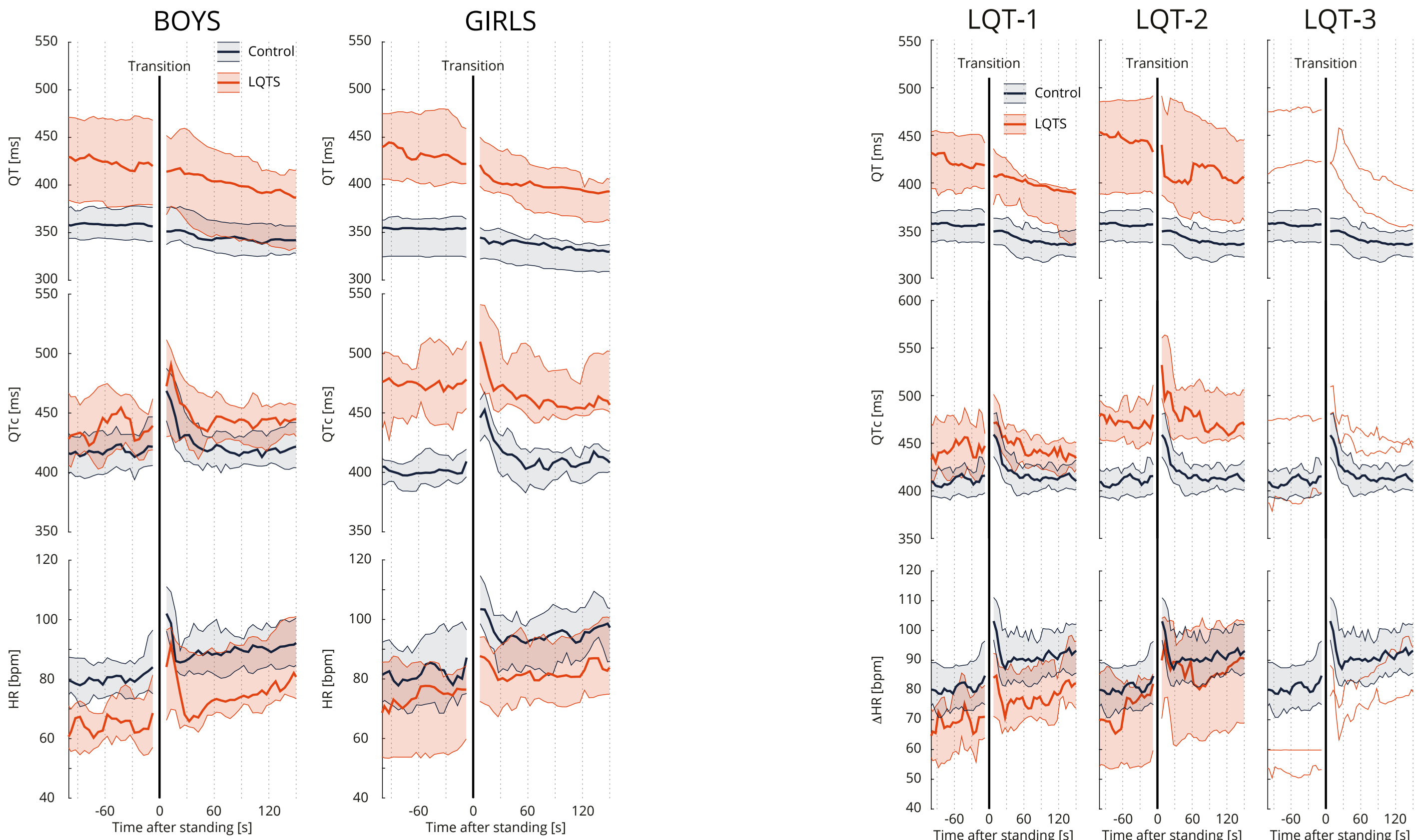

Figure S.5-2. Genotype differences in dynamics of the standing test. Including 14 LQT-1, 13 LQT-2 Figure S.5-1. Sex difference in dynamics of the standing test. Including 36 boys ( 25 controls and 11 the absolute QT-interval, QTc and heart rate of controls (blue) and LQTS-patients (orange), stratified for boys (left column) and girls (right column). Transition from supine to standing is indicated by the black solid line. HR= heart rate, LQTS= Long QT-syndrome, QTC= QT-interval corrected for heart rate using and 2 LQT-3 patients. Median and interquartile ranges of the absolute change of the QT-interval, QTC and heart rate to the baseline for controls and LQTS type 1 (LQT-1), LQTS type 2 (LQT-2), LQTS type 3 (LQT-3). Note that for LQT-3 we only showed the data of the 2 individual patients. Transition from supine to standing is indicated by the black solid line. HR= heart rate, LQTS= Long QT-syndrome, QTC= QTinterval corrected for heart rate using Bazett's formula. 


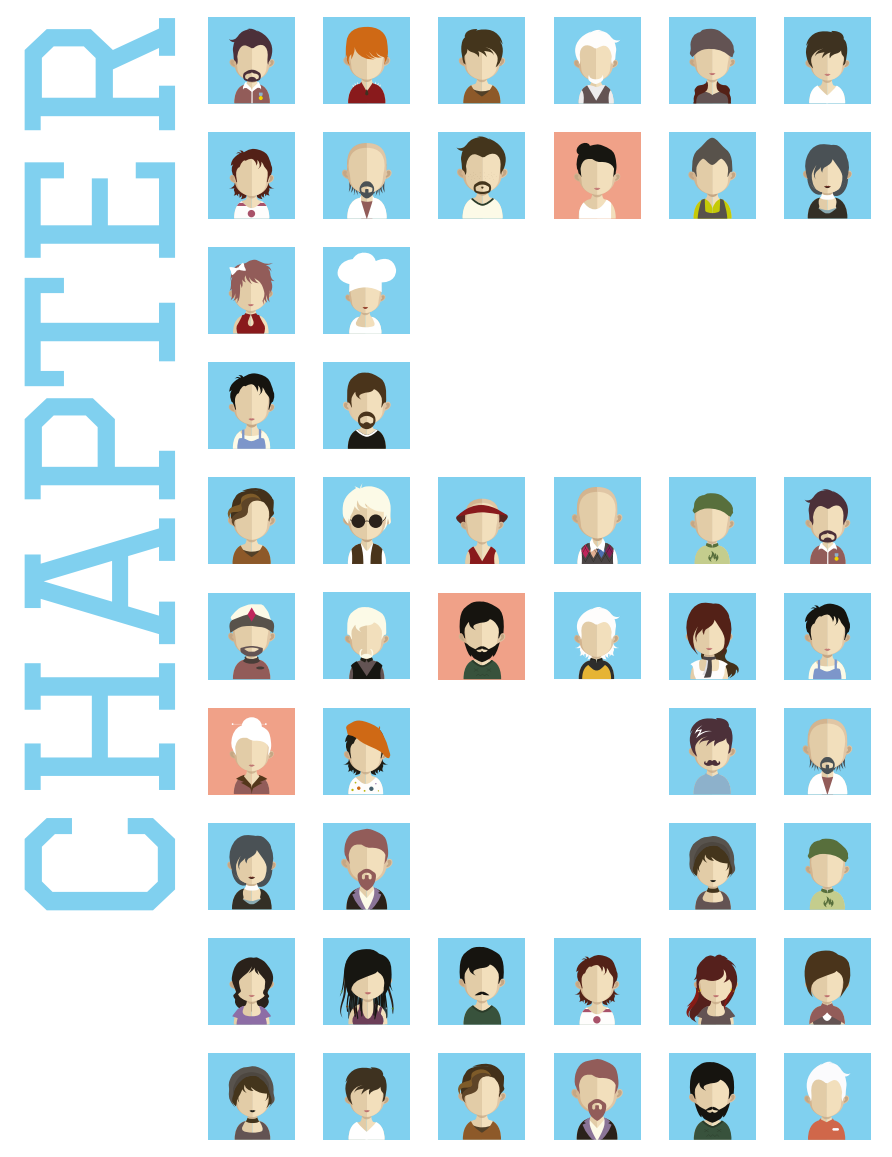

\section{CHAPTER 6}

SUPPORT VECTOR MACHINE-BASED ASSESSMENT OF THE T-WAVE MORPHOLOGY IMPROVES LQTS DIAGNOSIS 
Abstract

\section{Background}

Diagnosing long-QT syndrome (LQTS) is challenging due to a considerable overlap of the QTC-interval between LQTS patients and healthy controls.

\section{Objective}

The aim of this study was to investigate the added value of T-wave morphology markers obtained from 12-lead ECGs in diagnosing LQTS in a large cohort of gene-positive LQTS patients and genenegative family members using a support vector machine.

Methods

A retrospective study was performed including 688 digital 12-lead ECGs recorded from genotypepositive LQTS patients and genotype-negative relatives at their first visit. Two models were trained and tested equally: a baseline model with age, gender, RR-interval, QT-interval and QTc-intervals as inputs and an extended model including morphology features as well.

\section{Results}

The best performing baseline model showed an area under the receiver operating characteristic curve (AUC) of 0.821 , whereas the extended model showed an AUC of 0.901 . Sensitivity and specificity at the maximal Youden's indexes changed from 0.694 and 0.829 with the baseline mode to 0.820 and 0.861 with the extended model. Compared to clinically used QTC-interval cut-off values (>480ms), the extended model showed a major drop in false negative classifications of LQTS patients.

\section{Conclusion}

The support vector machine-based extended model with T-wave morphology markers resulted in a major rise in sensitivity and specificity at the maximal Youden's index. From this, it can be concluded that the T-wave morphology has an added value in the diagnosis of LQTS.

\subsection{Introduction}

Long-QT syndrome (LQTS) is an inheritable disease entity associated with malignant arrhythmias at young age. The diagnosis of congenital LQTS, once based on a scoring system of clinical and electrocardiographic parameters, is nowadays aided by genetic testing. Despite the fact that genetic testing is currently relatively easy to perform at relatively low costs, it remains of utmost importance to preserve genetic testing to persons suspected for LQTS, since distinguishing pathogenic variants from innocuous rare variants can be very complex'. To identify persons suspected for LQTS, genderbased cut-off values for a prolonged QT-interval corrected for heart rate (QTC) are used. However, it is known that there is a considerable overlap in QTc between LQTS patients and healthy controls², which hampers the accuracy of diagnosing LQTS based on QTc. Therefore, attempts have been made to take advantage of the fact that LQTS patients often display abnormal responses to heart rate changes. This led to diagnostic interventions in which the QT-interval adaptation to heart rate changes is studied in for example the exercise recovery phase ${ }^{3}$, after epinephrine provocation ${ }^{4}$ and the brisk-standing-test ${ }^{5}$. Although these interventions are known to improve LQTS diagnosis, a clinician should have an LQTS suspicion before such a test will be performed. LQTS patients with a normal QTC on the resting ECG are likely to never be tested with such an interventional diagnostic test when they are not overt symptomatic or become part of a family evaluation for LQTS and may therefore remain undiagnosed. Still, they may have exaggerated risks for malignant arrhythmias under particular conditions such as the use of certain drugs and they may have children who can become severely symptomatic when undiagnosed ${ }^{6}$.

Apart from a prolongation of QTc, variations in the morphological configuration of the T-wave are seen in LQTS patients, especially in relation to the LQTS type ${ }^{7,8}$ Previous studies have indicated that using T-wave morphologies during interventions as an additional marker to diagnose LQTS improves the diagnosis,10. These studies however not only require an intervention but their manually assessment of T-wave morphologies makes the results clinician dependent.

The added value of T-wave morphology markers in standard 10-seconds 12-lead ECGs to identify possibly LQTS genotype-positive patients of the three most common mutated genes has not yet been investigated. In this study we use a machine-learning approach to investigate the added value of T-wave morphology markers obtained from baseline 10-seconds 12-lead body surface ECGs to diagnose LQTS in a large cohort of gene-positive LQTS patients and gene-negative family members.

\subsection{Methods}

\subsubsection{Study population}

A retrospective cohort study consisting of LQTS patients and their family was performed. LQTS patients with LQTS type 1 (LQT1), type 2 (LQT2) and type 3 (LQT3) were confirmed by pathogenic variants in KCNQ1, KCNH2 or SCN5A respectively. All genotype-negative family members were used as healthy controls. All individuals were seen in the Academic Medical Centre in Amsterdam, The Netherlands, between January 1996 and December 2016. Inclusion criteria for this study were an age $\geq 16$ years, known genetic testing results and digitally available ECG at first presentation. Exclusion criteria were the presence of any comorbidity that might affect ventricular re- and/or depolarization. The study was 
approved by the Academic Medical Center Review Board and informed consent of the individuals was waived as this study used retrospective data from regular care.

\subsubsection{Electrocardiograms}

Digital standard 10-seconds 12-leads body surface ECGs performed in the initial evaluation of individuals in the work-up during (family) screening for LQTS were collected. ECGs were excluded when the ECG contained too much noise and when all T-waves in all ECG leads were too flat $(<40 \mu \mathrm{V})$ to reliably assess the QT-interval and T-wave morphology automatically. To avoid subjective evaluation of the T-wave morphology, all ECG landmarks and T-wave morphology features were calculated automatically using custom-made software in MATLAB (2017a, Mathworks, Natick, MA, USA).

\subsubsection{Data acquisition and pre-processing}

ECGs were stored in the MUSE Cardiology Information system (GE Healthcare, Little Chalfont, UK) and recorded with a 250 or $500 \mathrm{~Hz}$ sample frequency. All further processing and analyses in this study were done using custom-made software in MATLAB. ECGs were first filtered using a 2nd order bidirectional Butterworth band pass filter $(0.5-125 \mathrm{~Hz})$ and a 2 nd order infinite impulse response notch filter $(50 \mathrm{~Hz})$. For all individual ECG leads, the residuals of a median filter with a $600 \mathrm{~ms}$ window were regarded as baseline deviations and were therefore subtracted from the individual leads to correct for baseline wander. After filtering, a 1D Fourier up-sampling method was used to up-sample all ECG data to $1 \mathrm{kHz}$ to assure sampling frequency independency of our analysis.

\subsubsection{Average complex construction}

To obtain the best signal-to-noise ratio, analysis was done on average complexes as constructed for all individual ECG leads. To construct these average complexes, first all R-peaks were detected in the ECG lead with the highest R-peak amplitude using a modified Pan-Tompkins algorithm.11 From these R-peaks, a trimmed mean RR-interval was calculated after omitting $10 \%$ of the outermost RR-intervals. The individual complexes were selected from the R-peak location minus 25\% of the trimmed mean RR-interval to the R-peak plus 70\% of the trimmed mean RR-interval. All complexes were aligned on the R-peak and an average complex was calculated. Thereafter, to guarantee averaging of only reliable complexes, individual complexes with a correlation coefficient below 0.9 when correlated with the average complex as well as complexes with an RR-interval deviating more than $20 \%$ from the mean RR were excluded. If less than $60 \%$ of all complexes in the whole ECG were preserved after these exclusions, the entire ECG was excluded for further analysis. Furthermore, if less than $60 \%$ of all complexes in an individual lead were preserved, the entire lead was not taken into account since no reliable average complex could be constructed for this lead. Finally, if a complex was excluded in more than 3 leads, the complex was excluded for all leads to guarantee exclusion of e.g. ventricular extra systoles into the average complex. A new and final average complex for all remaining ECGs and leads was constructed from all remaining complexes. These final average complexes were used for further analysis.
Absolute

Length

Bip

Amplitude

Time to onset ${ }^{8}$ Height of the highest absolute value of the T-wave VR, VL, VF, V1-V6

\begin{tabular}{|c|c|c|}
\hline Time to onset ${ }^{8}$ & Interval between $R$ and Tstart & $\mathrm{ECG}_{\text {RMs }}$ and $P C A_{1}$ \\
\hline Skewness 14 & Degree of symmetry & $V R, V L, V F, V 1-V 6$ \\
\hline
\end{tabular}

Kurtosis ${ }^{14} \quad$ Degree of peakedness $\quad$ VR, VL, VF, V1-V6

$\begin{array}{lll}\text { Notch score }^{14} & \text { Derived from Andersen et al. } \\ \text { AR, VL, VF, V1-V6 }\end{array}$

Asymmetry score $^{14} \quad$ Derived from Andersen et al. $\quad$ VR, VL, VF, V1-V6

QRS amplitude'15 Height of the highest absolute value of the QRS-complex VR, VL, VF, V1-V6

\begin{tabular}{|c|c|c|}
\hline 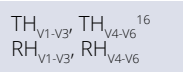 & $\begin{array}{l}\text { T-wave and R-peak heterogeneity = max }(V(v a r(X)) \\
\text { in which } X \text { is an } n \text {-by-3 matrix consistsing of three ECG leads of length } n \text {. }\end{array}$ & $\begin{array}{l}\mathrm{V} 1-\mathrm{V} 3 \\
\mathrm{~V} 4 \mathrm{~V} 6\end{array}$ \\
\hline SP QRS-T angle & $\begin{array}{l}\text { Spatial peak QRS-T angle is the smallest angle between the vector at } \\
\text { maximal T-wave magnitude and the vector at maximal QRS complex }\end{array}$ & VCG \\
\hline
\end{tabular}

maximal T-wave magnitude and the vector at maximal QRS complex
magnitude in the VCG

$\begin{array}{ll}\text { SM QRS-T angle }{ }^{17} & \begin{array}{l}\text { Spatial mean QRS-T angle is the smallest angle between the mean vector of } \\ \text { the T-wave and the mean vector of the QRS-complex in the VCG }\end{array}\end{array}$

$T_{\text {peak }} T_{\text {end }}$ interval ${ }^{5} \quad$ Interval between $T_{\text {peak }}$ and $T_{\text {eat }}$ $\mathrm{PCA}_{1}$

Table 6-1 T-wave morphology features summary. $\mathrm{TH}=\mathrm{T}$-wave heterogeneity, $\mathrm{RH}=\mathrm{R}$-peak heterogeneity, SP = spatial peak, SM = spatial mean, $\mathrm{VCG}=$ vectorcardiogram, $\mathrm{PCA} \mathrm{A}_{1}=$ the first component of principal component analysis, ECGRMS = root mean square of the unipolar ECG leads.

\subsubsection{Landmark detection}

To detect a global R-peak and QRS-onset, a root mean square ECG (ECGRMS) was constructed from the precordial leads and the reconstructed unipolar leads VR, VL, VF as described previously by our research group. ${ }^{12}$ The first component of principal component analysis (PCA $)$ using singular value decomposition on the unipolar ECG leads (VR, VL, VF and V1-V6) from R-peak + 95ms to R-peak + 0.7xRR was used for global T-wave landmarks. The peak of the T-wave $\left(T_{\text {peak }}\right)$ was detected as the most prominent peak of PCA. The start and end of the T-wave ( $T_{\text {sat }}$ and $T_{\text {end }}$ ) were detected by means of an automated tangent method as we described earlier. ${ }^{12} T_{\text {start }}$ and $T_{\text {end }}$ were manually checked by one observer (TD) since multiple T-wave morphology features rely on a proper determination of $T_{\text {start }}$ and $T_{\text {end. }}$.

\subsubsection{T-wave morphology features}

All T-wave morphology features, apart from the T-wave heterogeneities, were computed within the window $T_{\text {start }}$ and $T_{\text {end }}$ for all unipolar ECG leads. Most of the features were calculated for all ECG leads individually. For some features, the $\mathrm{ECG}_{\mathrm{RMS}}, \mathrm{PCA}$ or a vectorcardiogram (VCG) (reconstructed using the Kors matrix ${ }^{13}$ ) were used. All computed features are listed in Table 6-1. For a more detailed description of the T-wave morphology features, see Supplementary materials. 


\subsubsection{Support vector machine}

\section{Model inputs}

Subjects were classified as gene-positive or gene-negative by a machine learning classification model based on multiple inputs. Missing feature values were replaced by random values within mean \pm standard deviation for the corresponding feature. Two models were created: a baseline model with age gender, RR-interval, QT-interval and QTC (QT corrected for heart rate by the correction formulas of Bazett Fridericia, Framingham and Hodges ${ }^{18}$ ) as inputs and an extended model with all morphology features as additional model inputs. The performance of the baseline model was used to determine the optima classification using commonly used clinical parameters. The difference between the baseline model and the extended model demonstrates the added diagnostic value of T-wave morphology features.

\section{Model training and testing}

The model and training used in the current study are summarized in Figure 6-1. Both the baseline and the extended model were support vector machine models with a linear kernel and were trained and tested on the ECGs of the entire cohort using cross-validation. Therefore, the performance of these models cannot be assessed directly. Hence, to investigate the performances of the baseline and extended models, 100 similar models were trained and tested on different randomized training and testing sets. This was done for both the baseline and extended model. Training sets consisted of a randomly chosen subset of ECGs containing 90\% of all individuals whereas testing sets consisted of the ECGs of the remaining $10 \%$ of all individuals. The mean performance of these 100 models is the expected performance of the final model. ${ }^{19}$

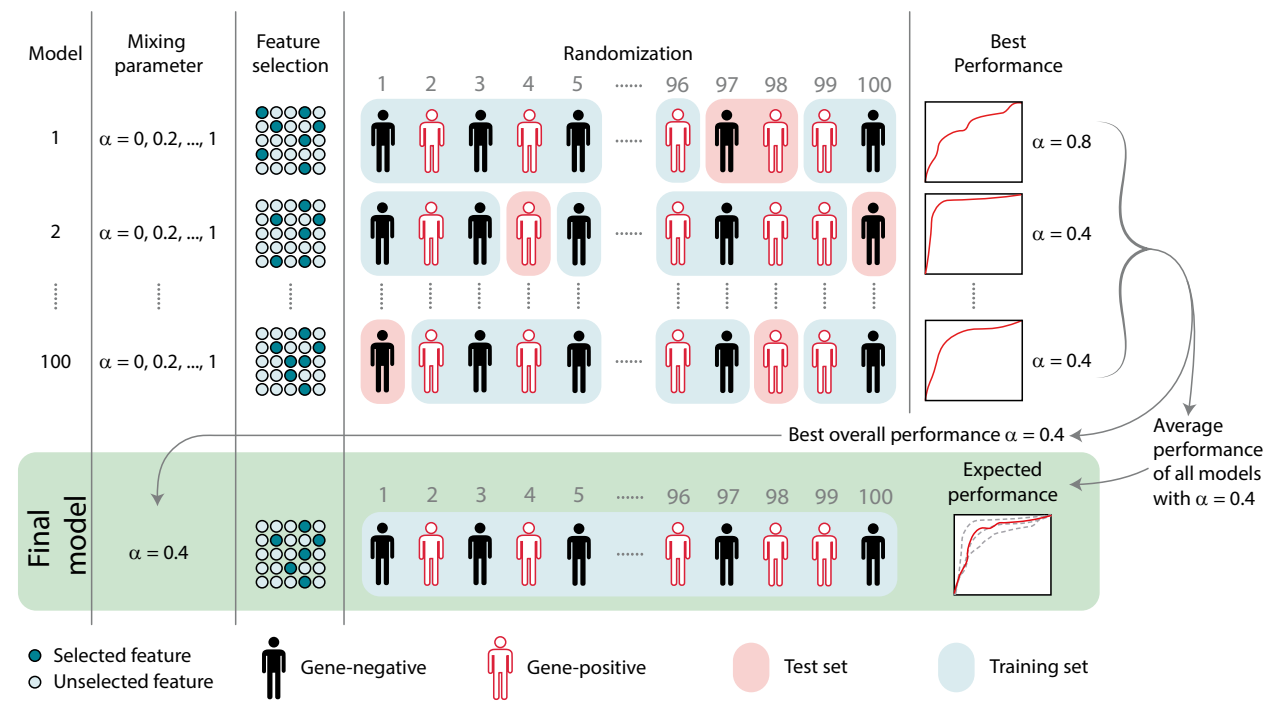

Figure 6-1 Schematic representation of the machine learning steps. A detailed description is given in the main text. $a=$ mixing parameter.
For each of these 100 models, features with the highest discriminative performance were selected by means of elastic net regularization, combined with maximum likelihood estimation in a logistic regression model. For a detailed description of elastic net regularization, we refer to Zou and Hastie. ${ }^{20}$ In short, it combines two feature selection methods (lasso and ridge regression). The mixing parameter (a) controls the ratio of both feature selection methods to obtain an optimal combination. The tuning parameter $(\lambda)$ controls the strength of this optimal combination. For each of the 100 models, during feature selection the value of a was varied from 0 to 1 , with steps of 0.2 . For each value of $a, 100$ values of $\lambda$ ranging from $\lambda_{\max }$ (at which all features were excluded) to $10^{-4} \cdot \lambda_{\max }$ were tested. ${ }^{21}$ For each $\lambda$, the cross-validated error resulting from 10 -fold cross-validation on the current training set was noted. Subsequently, for each a selected features resulting from the cross-validation were noted at $\lambda_{\min }$ (at the minimal cross-validated error) and $\lambda_{1 S E}\left(\lambda_{\min }+1\right.$ standard error).

For each of the 100 models, the AUC was calculated with each $a$ at $\lambda_{\min }$ and $\lambda_{15 E}$. The combination of a and $\lambda$ with the highest mean AUC over all 100 models was selected as the optimal combination. This $a$ and $\lambda$ were used in the final model.

\subsubsection{Statistical analysis}

Values are expressed as mean \pm standard deviation. The differences between LQTS patients and genotype-negative family members were analysed by independent samples T-test for parametric data, and chi-square for non-parametric data. A p-value $<0.05$ was considered statistically significant. Receiver-operating characteristic (ROC) analysis was used to determine the performance of the models. Optimal performance was the point with the maximal Youden's index ( $Y$ I =sensitivity+specificity-1). The area under the curve $(A \cup C), Y_{\text {max }}$ the sensitivity and specificity were used to quantify the ability of the different models to diagnose LQTS.

\subsection{Results}

\subsubsection{Study population}

Of all 1087 individuals with digitally available ECGs in their work-up during (family) screening for LQTS, 284 were $<16$ years of age and for 48 individuals genetic testing results were unknown. From the remaining 755 individuals eligible for the study, 45 (6.0\%) were excluded based on the presence of comorbidities that might potentially affect ventricular re- and/or depolarization (varying from e.g. bundle branch blocks, hypokalaemia, thalassemia, angina pectoris, an overlap syndrome with Brugada syndrome, to severe post-anoxic encephalopathy). In 11 (1.5\%) individuals the ECG registration contained too much noise and in $6(0.8 \%)$ individuals T-waves were too flat ( $\angle 40 \mathrm{DV}$ ) to reliably calculate $\mathrm{T}_{\text {sen }}$ and $\mathrm{T}_{\text {.nd }}$. In 5 $(0.7 \%)$ individuals a correct ECG export failed. The remaining $688(91.1 \%)$ individuals were included in the analyses. The baseline characteristics of the LQTS patients and the genotype-negative family members are shown in Table 6-2. LQTS patients were statistically significant younger compared to controls $(41 \pm 15$ versus $45 \pm 15$ years, $p<0.001$ ). 
Number [-]

Gender ( $\mathrm{m} / \mathrm{f})$

Age [years]

$\begin{array}{ll} & \text { Num } \\ \text { Control } & 348 \\ \text { LQTS } & 340 \\ \text { LQT1 } & 129 \\ \text { LQT2 } & 160 \\ \text { LQT3 } & 51\end{array}$

$163 / 185$

$143 / 197$

$41 \pm 15$

$52 / 77$

$72 / 88$

$19 / 32$

$42+15$

$42 \pm 15$

Table 6-2 Study population characteristics. Age is given as mean \pm standard deviation. All others are presented as counts. LQTS = long QT syndrome, LQT1, LQT2 and LQT3 = long QT syndrome type 1, 2 and 3 .

\subsubsection{Baseline model}

The best performing support vector machine-based baseline model was reached with an a of 1 and an elastic net tuning parameter of $\lambda \mathrm{min}$. The selected features for the baseline model were: age, QT-interval and QTC-Hodges. The ROC of the best performing baseline model is shown in Figure 6-2. ROC analysis resulted in an AUC of 0.821 and a YImax of 0.523 with a corresponding sensitivity and specificity of 0.694 and 0.829 respectively.

\subsubsection{Extended model}

The best performing support vector machine-based extended model with additional morphology inputs was reached with an a of 0.2 and an elastic net tuning parameter of $\lambda 1 \mathrm{SE}$. ROC curves, shown in Figure 6 2, resulted in an AUC of 0.901 (95\% Cl 0.893-0.909), a YImax of 0.681 and a sensitivity and specificity of 0.820 and 0.861 respectively. All selected features along with their coefficients are listed in Table 6-3. Compared to the baseline model, AUC, sensitivity and specificity increased with $0.080,0.126$ and 0.032 respectively.

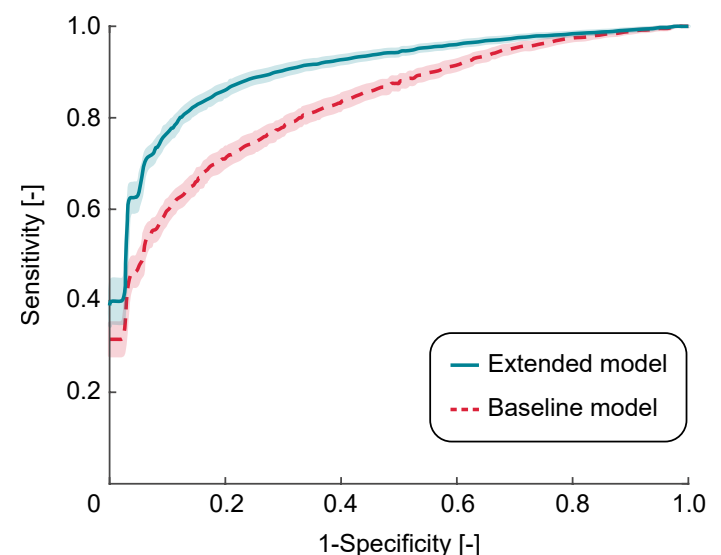

Figure 6-2 Average receiver operating characteristic curves with $95 \%$ confidence intervals of the extended model (solid turquoise) and the baseline model (dashed red).

\begin{tabular}{|c|c|}
\hline Feature & $\beta$ \\
\hline Age & -0.010 \\
\hline QT & 0.002 \\
\hline QTc Bazett & 0.0048 \\
\hline QTc Fridericia & 0.0044 \\
\hline QTc Framingham & 0.0043 \\
\hline QTc Hodges & 0.0048 \\
\hline Area in $\mathrm{VL}, \mathrm{V} 2, \mathrm{~V} 3$ & $3.57 \times 10^{-6}, 1.69 \times 10^{-7}$ and $1.96 \times 10^{-6}$ \\
\hline Absolute area in VL, V1, V2, V3 & $2.28 \times 10^{-6}, 1.35 \times 10^{-5}, 1.45 \times 10^{-6}$ and $4.13 \times 10^{-6}$ \\
\hline Biphasicness in VR, V1, V2, V6 & $-0.51,-0.09,-0.08$ and -0.35 \\
\hline Amplitude in VR, VF, V6 & $5.49 \times 10^{-4},-1.54 \times 10^{-5}$ and $-1.35 \times 10^{-4}$ \\
\hline Length & 0.011 \\
\hline Time to onset & 0.007 \\
\hline Skewness in VR, VL, VF, V4, V5, V6 & $-0.69 ;-0.24 ;-0.47 ;-0.07 ;-0.40 ;-0.21$ \\
\hline Kurtosis in VL and $\mathrm{V} 3$ & $0.43 ; 0.30$ \\
\hline Asymmetry in VR, VL VF, V2, V3 & $1 ; 0.09 ; 0.07 ; 0.03 ; 0.21$ \\
\hline Tpeak to $T_{\text {end }}$ interval & 2 \\
\hline QRS amplitude VL, VF, V1 & $-1.40 \times 10^{-4},-2.99 \times 10^{-4}, 6.19 \times 10^{-5}$ \\
\hline SP QRS-T angle & 0.0018 \\
\hline $\mathrm{RH}_{\mathrm{V} 4 \mathrm{~V} 6}$ & $-3.00 \times 10^{-4}$ \\
\hline $\mathrm{TH}_{\mathrm{v} 4 \mathrm{v} 6}$ & 0.0012 \\
\hline
\end{tabular}

Table 6-3 Selected features. Note that all features are unitless because all features are normalised by subtracting the mean and dividing the result by the standard deviation (with mean and standard deviation determined from the training set). $\mathrm{TH}$ = T-wave heterogeneity, $\mathrm{RH}$ = R-peak heterogeneity, SP = spatial peak.

\subsubsection{Clinical QTc cut-off value}

Figure 6-3 shows the amount of correctly and incorrectly classified cases and controls based on the clinically used QTc-Bazett thresholds $\left(>480 \mathrm{~ms}^{22}\right)$ as well as the classification results based on the extended model. As shown in Figure 6-3, the extended model resulted in a major drop of incorrectly classified LQTS patients. It can also be seen that some controls with a QTc $>480 \mathrm{~ms}$ are correctly classified as control by the extended model. This all comes at the cost of some controls (with QTc < $480 \mathrm{~ms}$ ) being incorrectly classified as LQTS patients. 


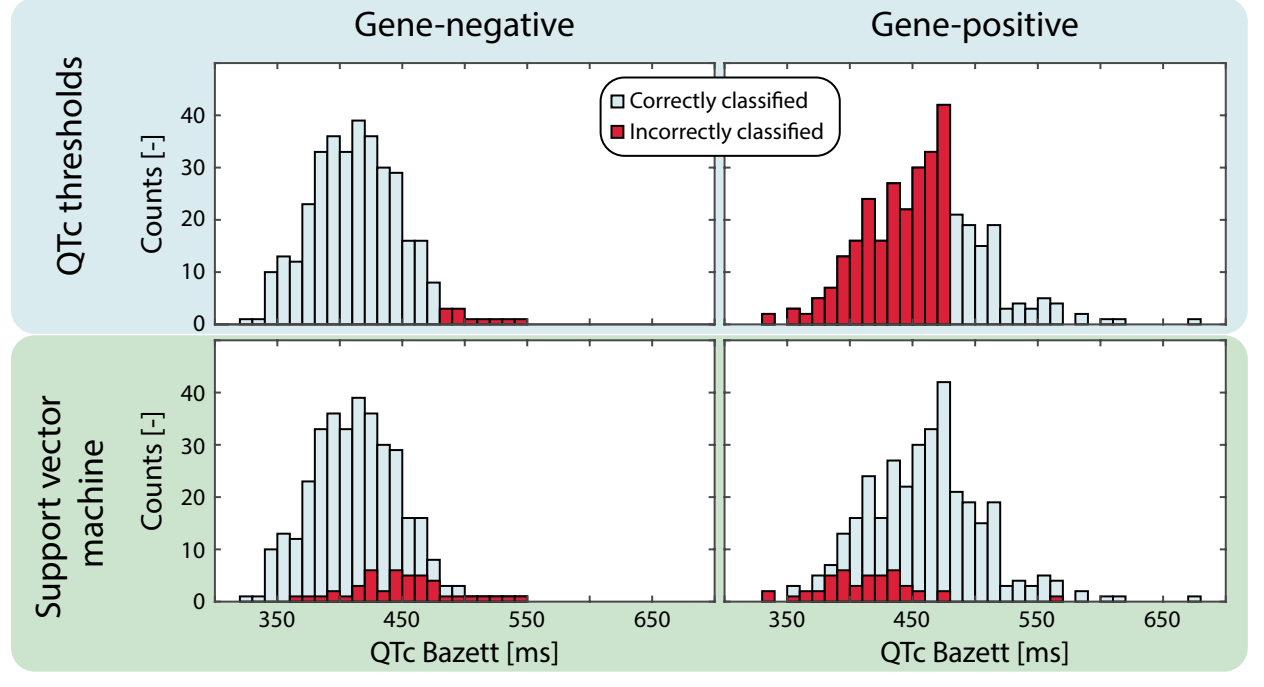

Figure 6-3 Graphic representation of the added value of the support vector machine with T-wave morphology features on clinically used QTc thresholds. The amount of correctly and incorrectly classified gene-negative subjects (left) and gene-positive subjects (right) based on QTc cut-off value of $480 \mathrm{~ms}$ (top panel) and based on our final support vector machine (bottom panel). The classifications as shown in this figure are the average classifications for each individual patient at YImax obtained from 1000 Monte Carlo cross-validations.

\subsection{Discussion}

In the current study, we compared a baseline model using age, gender, QTc and QT-interval as inputs with a model extended with T-wave morphology inputs to investigate the added value of T-wave morphology markers in the automated diagnosis of LQTS. Comparison of the ROC analyses of both models showed that the model extended with T-wave morphology markers resulted in a better performance. Since both models were trained and tested similarly, this improved performance can be attributed to the addition of the T-wave morphology markers. In other words, it can be concluded that T-wave morphology markers have an added value to age, gender, QTc and QT-interval in automatically distinguishing LQTS patients from genotype-negative family members.

The sensitivity and specificity of respectively 0.820 and 0.861 from our extended model might seem modest to previous studies, which have suggested higher performances when using T-wave markers for the diagnosis of LQTS. ${ }^{9114,23,24}$ However, our study population consisted of LQTS patients and genenegative family members whereas other studies used healthy individuals as controls. ${ }^{9114,23,24}$ In our study, QTc of the control group showed considerable more overlap with QTc of LQTS patients than in the above-mentioned studies. Therefore, distinguishing gene-positive LQTS from gene-negative family members is more challenging than distinguishing LQTS patients from healthy controls. This might explain the higher performance of other studies on the added value of T-wave morphology markers to identify LQTS patients. Beside the use of different control groups, the ECG recordings also differed between our study and the studies by Immanuel et al..$^{23}$ and Chorin et al. ${ }^{9}$ who respectively studied 24h Holter registrations and T-wave morphologies during the brisk-standing-test. The current study only used standard 10-second 12-leads body surface ECGs but could nevertheless show that T-wave morphology markers in these ECGs improve prediction results. Since these standard ECGs are widely available and are part of clinical routine, the added value of being able to improve LQTS diagnosis from these recordings is considerable.

In this study population, gene-negative family members were found to be significantly older than LQTS patients. Not surprisingly, age was a selected feature in both the baseline and the extended model and contributed to the performances of both models. However, since age was a selected feature in both models, the effect of age on the performance of the models is expected to be the same. Therefore, the comparison between both models is still valid.

Next to the support vector machine, a logistic regression model and a bagged random forest were used From these methods, the support vector machine resulted in the best mean discriminative performance and was therefore used as the machine learning method in this study.

Although it has been shown that Hodges' QTc is less correlated with heart rate compared to the others, ${ }^{18}$ still QTC Bazett is the most commonly used QTc correction method. However, the fact that the only QTC method selected in our baseline model was QTc Hodges might be another argument that the clinical use of QTc Hodges should be re-evaluated.

\subsubsection{Limitations}

Although the results of this study are promising, there is still room for improvement. First of all, no notch scores are used in our models. Initially, we implemented the notch score as described by Andersen et al. ${ }^{14}$ However we found a very poor agreement between the implemented notch score and visual inspection by two blinded observers. We therefore chose to exclude this feature from all models. Secondly, $T_{\text {star }}$ or $\mathrm{T}_{\text {end }}$ have been manually adjusted for 30 out of $688 \mathrm{ECGs}$. Although the algorithm seems to work for the majority of ECGs, improving these steps might be necessary before our algorithm can be used on a arger scale. A third potentially important limitation of the methodology used in this study is that too flat T-waves ( $<40 \mu \mathrm{V}$ ) have to be excluded even though flat T-waves are a specific aberrant T-wave morphology feature in LQTS patients.Next, the signal quality of these retrospectively collected ECGs was relatively poor for the current study purpose in relatively many cases. Since all ECGs were recorded because of clinical routine, no special care was taken to obtain very high-quality ECGs. Though the relatively poor ECG quality might be an explanation for the two limitations described above, future directions should be to increase the robustness of the model to deal with ECGs from daily clinical routine. 


\subsection{Conclusion}

In the current study, we compared a baseline and an extended model including T-wave morphology inputs, both using support vector machines, to investigate the added value of T-wave morphology markers in diagnosing LQTS. The performances of both models showed that the model extended with T-wave morphology markers resulted in a better performance in the diagnosis of LQTS. Therefore, it can be concluded that the use of T-wave morphology markers has an added value to distinguish LQTS patients from genotype-negative family members.

\subsection{References}

1. Wilde, A. A. M. \& Ackerman, M. J. Exercise extreme caution when calling rare genetic variants novel arrhythmia syndrome susceptibility mutations. Heart Rhythm 7, 1883-1885 (2010).

2. Viskin, S. The QT interval: Too long, too short or just right. Heart Rhythm 6, 711-715 (2009).

3. Sy, R. W. et al. Derivation and validation of a simple exercise-based algorithm for prediction of genetic testing in relatives of LQTS probands. Circulation 124, 2187-2194 (2011).

4. Ackerman, M. J. et al. Epinephrine-Induced QT Interval Prolongation: A Gene-Specific Paradoxical Response in Congenital Long QT Syndrome. Mayo Clin. Proc. 77, 413-421 (2002).

5. Viskin, S. et al. The Response of the QT Interval to the Brief Tachycardia Provoked by Standing. J. Am. Coll. Cardiol. 55, 1955-1961 (2010).

6. Postema, P. G. et al. Safe drug use in long QT syndrome and Brugada syndrom. Europace 15 1042-1049 (2013)

7. Lehmann, M. H. et al. T wave 'humps' as a potential electrocardiographic marker of the long QT syndrome. J. Am. Coll. Cardiol. 24, 746-754 (1994).

8. Moss, A. J. et al. ECG T-Wave Patterns in Genetically Distinct Forms of the Hereditary Long QT Syndrome. Circulation 92, 2929-2934 (1995).

9. Chorin, E. et al. Diagnostic value of T-wave morphology changes during "QT stretching" in patients with long QT syndrome. Heart Rhythm 12, 2263-2271 (2015)

10. Khositseth, A., Hejlik, J., Shen, W. K. \& Ackerman, M. J. Epinephrine-induced T-wave notching in congenital long QT syndrome. Heart Rhythm 2, 141-146 (2005).

11. Pan, J. \& Tompkins, W. J. A real-time QRS detection algorithm. IEEE Trans. Biomed. Eng. 32, 230-6 (1985).

12. Hermans, B. J. M. et al. The development and validation of an easy to use automatic QT-interval algorithm. PLoS One 12, e0184352 (2017)

13. Kors, J. A., van Herpen, G., Sittig, A. C. \& van Bemmel,J. H. Reconstruction of the Frankvectorcardiogram from standard electrocardiographic leads: diagnostic comparison of different methods. Eur. Heart J. 11, 1083-1092 (1990).

4. Andersen, M. P. et al. A robust method for quantification of IKr-related T-wave morphology abnormalities. in 2007 Computers in Cardiology 34, 341-344 (IEEE, 2007)

15. Mincholé, A., Ariga, R., Neubauer, S., Watkins, H. \& Rodr, B. Electrocardiographic abnormalities of hypertrophic cardiomyopathy. Comput. Cardiol. (2010). 41, 397-400 (2014).

16. Tan, A. Y. et al. Interlead heterogeneity of R- and T-wave morphology in standard 12-lead ECGs predicts sustained ventricular tachycardia/fibrillation and arrhythmic death in patients with cardiomyopathy. J. Cardiovasc. Electrophysiol. 28, 1324-1333 (2017).

7. Oehler, A., Feldman, T., Henrikson, C. A. \& Tereshchenko, L. G. QRS-T Angle: A Review. Ann. Noninvasive Electrocardiol. 19, 534-542 (2014).

8. Luo, S., Michler, K., Johnston, P. \& MacFarlane, P. W. A comparison of commonly used QT correction formulae: The effect of heart rate on the QTc of normal ECGs. J. Electrocardiol. 37, 81-90 (2004).

19. James, G., Witten, D., Hastie, T. \& Tibshirani, R. An Introduction to Statistical Learning. An introduction to statistical learning: with applications in R 103, (Springer New York, 2013).

20. Zou, H. \& Hastie, T. Regularization and variable selection via the elastic-net. . . R. Stat. Soc. 67, $301-$ 320 (2005).

21. Friedman, J., Hastie, T. \& Tibshirani, R. Regularization Paths for Generalized Linear Models via Coordinate Descent. J. Stat. Softw. 33, 1-22 (2010). 
22. Priori, S. G. et al. HRS/EHRA/APHRS Expert Consensus Statement on the Diagnosis and Management of Patients with Inherited Primary Arrhythmia Syndromes. Heart Rhythm 10, 1932-1963 (2013).

23. Immanuel, S. A. et al. T-wave morphology can distinguish healthy controls from LQTS patients. Physiol. Meas. 37, 1456-1473 (2016)

24. Sugrue, A. et al. Automated T-wave analysis can differentiate acquired QT prolongation from congenital long QT syndrome. Ann. Noninvasive Electrocardiol. 22, 1-7 (2017).

\subsection{Supplementary materials}

\section{T-wave morphology feature calculations}

Amplitude

To calculate the T-wave amplitude, both the most positive and the most negative peak in the signal were detected. The peak with the largest absolute value was noted as the T-wave amplitude.

\section{Area and absolute area}

The area was calculated as the integral of the T-wave amplitudes (with regard to its sign) between $T_{\text {star }}$ and $T_{\text {end }}$. The absolute area is calculated as the integral of the absolute T-wave amplitudes.

\section{Biphasicness score}

The biphasicness score of a T-wave was calculated by dividing the absolute value of the T-wave area by the absolute area of the T-wave and subtracting it from 1. For example, a negative T-wave with an are of -2 and an absolute area of 2 will have a biphasicness score of $1-(|-2| / 2)=0$. A biphasic T-wave that is $60 \%$ positive and $40 \%$ negative with an area of 0.4 and an absolute area of 2 will have a biphasicness score of $1-(|0.4| 2)=0.8$. In biphasic T-waves (defined as T-waves with a biphasicness score of $\leq 0.25$ ) no skewness, kurtosis, notch score and asymmetry score were calculated.

\section{Skewness and kurtosis}

For the calculation of the T-wave skewness and kurtosis, T-waves were treated as probability distribution curves and normalized between 0 and 1 before calculating the skewness and kurtosis.

\section{Notch and asymmetry scores}

Notch and asymmetry scores were derived from Andersen et al..$^{20}$ However, instead of calculating the notch score only in the first principal component of the signal, a notch was sought for in every lead.

To calculate the asymmetry score, first the first derivative of the ECG was split into a segment from $T_{\text {stat }}$ to $T_{\text {peak }}$ and a segment from $T_{\text {peak }}$ to $T_{\text {end }}$. Both segments were scaled between 0 and 1. Segment 2 was flipped over both the horizontal and vertical axis. Finally, the mean difference between the two segments was calculated as the asymmetry score.

\section{Spatial and mean peak QRS-T angle}

The spatial peak QRS-T angle is defined as the angle between the vector at maximal T-wave magnitude in the VCG and the vector at maximal QRS complex magnitude in the VCG. The spatial mean QRS-T angle is defined as the angle between the mean vector of the T-wave and the mean vector of the QRS-complex in the VCG.

\section{R-peak and T-wave interlead heterogeneity}

R-peak and T-wave inter-lead heterogeneity were calculated as described by Tan et al..$^{23}$ In summary: to calculate R-peak and T-wave heterogeneity, the QRS-complex and T-wave were selected in two groups of precordial leads (V1-V3 and V4-V6). The maximum value of the square root of the variance around the average of the three leads was calculated and used as the interlead heterogeneity. 


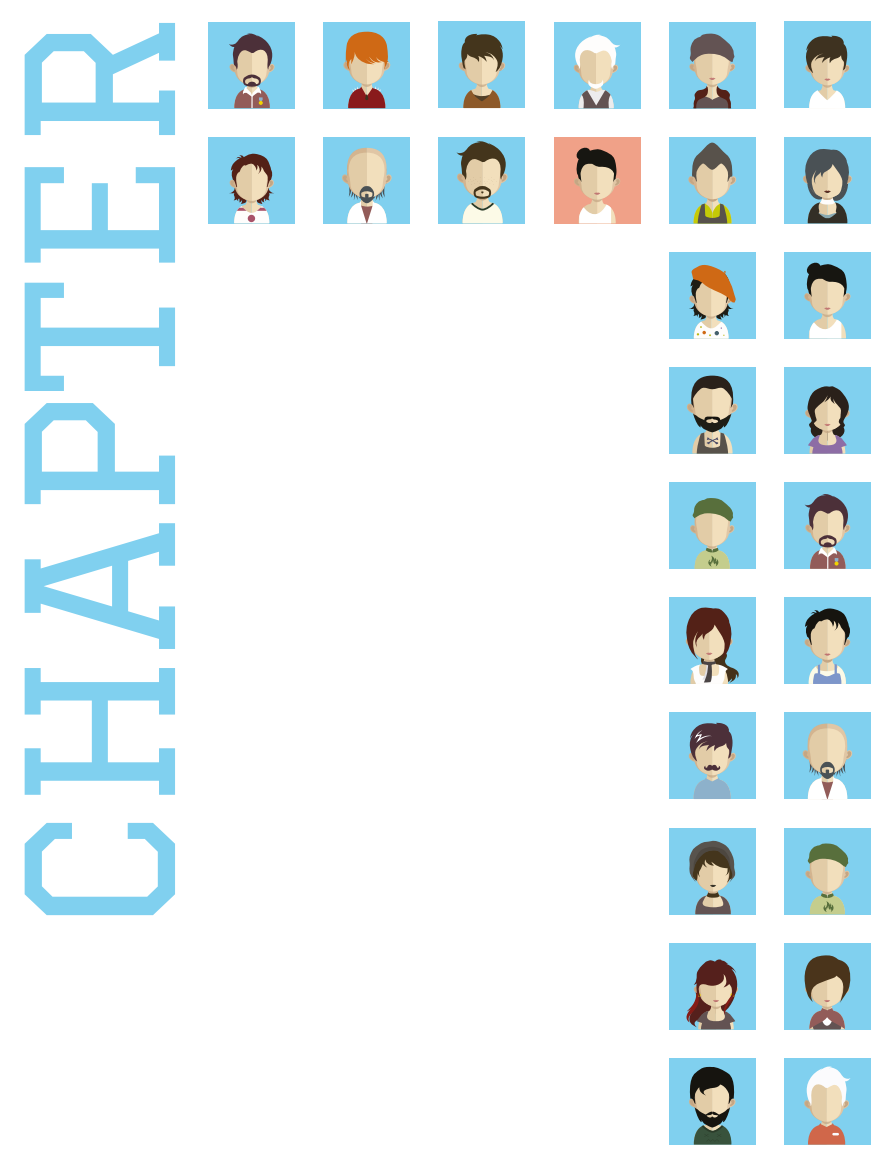

\section{CHAPTER 7}

IMPROVING LQTS DIAGNOSIS BY A POLYNOMIAL BASED T-WAVE MORPHOLOGY CHARACTERIZATION 
Abstract

\section{Background}

Diagnosing long-QT syndrome (LQTS) remains challenging due to a considerable overlap in QTinterval between LQTS and healthy subjects. Characterizing T-wave morphology might improve LTS diagnosis.

Objective

To improve LQTS diagnosis by combining new polynomial-based T-wave morphology parameters with the corrected QT-interval (QTc), age and sex in a model.

Methods

A retrospective cohort consisting of 333 LQTS patients and 345 genotype-negative family members was used in this study. For each patient, a linear combination of the first 2 Hermite-Gauss (HG) polynomials was fitted to the STT-segments of an average complex of all precordial leads and limb leads I and II. The weight coefficients as well as the error of the best fit were used to characterize T-wave morphology. Subjects were classified as LQTS or controls by clinical QTc cut-offs and three support-vector-machine (SVM) models fed with different features. An external cohort consisting of 72 patients and 45 controls was finally used to check the robustness of the models.

Results

Baseline QTc cut-offs were specific but had low sensitivity in diagnosing LQTS. The model with T-wave morphology features, QTc, age and sex had the best overall accuracy (84\%) followed by a model with QTc, age and sex (79\%). The Extended model especially performed better in LQT-3 patients (69\%).

Conclusion

T-wave morphologies can be characterized by fitting a linear combination of the first 2 HG polynomials. Adding T-wave morphology characterizations to age, gender and QTc in an SVM-mode improves LQTS diagnosis.

\subsection{Introduction}

Early diagnosis of congenital long-QT syndrome (LQTS) is crucial since early treatment can prevent malignant arrhythmias. Current guidelines recommend diagnosing LQTS based on either QT-interva corrected for heart rate (HR) using Bazett's formula (QTC), DNA testing, or the Schwartz-score ${ }^{1-3}$. These methods have some clinical limitations. First of all, diagnosing LQTS solely based on (sex specific) QTC cut-offs comes with some limitations. For example, the QT-interval seems hard to measure since the 95\% interobserver limits of agreement ranges up to $30 \mathrm{~ms}$ and even the interobserver variation between LQTS experts can measure up to 70ms $\mathrm{s}^{4.5}$. Furthermore, LQTS diagnosis is troubled because there is a considerable overlap in QTc between affected and unaffected subjects and QTc is variable over time 4,6,7. $^{\text {. }}$ Secondly, DNA-testing for known LQTS mutations also has limitations as approximately 20\% of clinically diagnosed LQTS patients remain genetically elusive and known LQTS mutations seem to have a reduced penetrance and therefore not always lead to clinical signs ${ }^{8,9}$. Furthermore, classification and reporting of potentially malign genetic variants might currently be incomplete and misleading ${ }^{10}$. Lastly, though a Schwartz-score of $\geq 3$ has a higher specificity in the diagnosis of LQTS compared to a baseline QTC cut-off of $\geq 430 \mathrm{~ms}$ ( $99 \%$ vs $86 \%$ ), this comes at the costs of a very low sensitivity (36\% vs $72 \%$ ) when genetic testing results are used as the true LQTS diagnosis ${ }^{11}$. Apart from these traditional tests, more sophisticated tests like individualized corrected QT-intervals ${ }^{12}$ and provocative tests that study QTinterval adjustment to changes in HR have been suggested ${ }^{13-19}$. A downside of these studies is that the suspicion for LQTS has to be raised before these tests will be used. Improved LQTS diagnosis based on standard ECGs would therefore still be useful.

Apart from a prolonged QTc, altered T-wave morphologies can be seen on LQTS patient's standard ECGs and could aid in diagnosing LQTS ${ }^{20-22}$. LQT-1 patients often show an early-onset broad T-wave, LQT-2 patients can have asymmetric, low voltage, biphasic and notched T-waves and LQT-3 patients typically have a late-onset T-wave. Although it is not strictly mentioned in the guidelines, LQTS experts will not only measure the QT-interval but also evaluate the T-wave morphology to diagnose LQTS ${ }^{23,24}$ Characterizing the T-wave morphology by eye-balling however is subjective and its diagnostic value depends on cardiologist's experience. Previous studies have shown that algorithm-based automatic assessment of the T-wave morphology can help in the diagnosis of LQTS ${ }^{25,26}$. Such algorithms might furthermore be useful to monitor treatment efficiency in diagnosed LQTS patients and by doing so can be useful in determining optimal patient specific treatments and drug doses.

The aim of this study was to improve diagnosis of LQTS based on standard ECGs by a machine-learning model with a new polynomial-based T-wave morphology characterization. After training and testing our approach on the cohort used in a previous report ${ }^{25}$ we pursued additional validation on an externa dataset. Lastly, the performance of our model was compared to the diagnostic accuracy of a QT-expert.

\subsection{Methods}

\subsubsection{Study population}

The same cohort (from now on called the Amsterdam data) as included in our earlier study was used in this study. A detailed description can be found in our previous study ${ }^{25}$. In short, this retrospective cohort from the Academic Medical Centre in Amsterdam (The Netherlands) consists of confirmed LQTS Type 1 
(LQT-1), Type 2 (LQT-2) and Type 3 (LQT-3) patients and their genotype-negative family members who are used as controls. Exclusion criteria were age $<16 y e a r s$, absence of genetic testing results, absence of baseline data and all other pathologies or medications that affect T-wave morphology. According to the Dutch Act on Medical Research involving Human Subjects, written informed consent was not necessary to obtain for the present study, due to the observational design of the study.

A retrospective cohort from the University Hospital Leuven (Belgium) was used as an additional external test set (from now on called the Leuven data). Similar to the Amsterdam data, the LQTS patient group consists of LQT-1, LQT-2 and LQT-3 and the control group consists of genotype-negative family members. Many patients from this cohort were included in a previous study ${ }^{12}$. The research protocol was approved by the local ethics committee. The same exclusion criteria were used for the Leuven data.

\subsubsection{ECG analysis}

\section{Acquisition and pre-processing}

All standard ECGs were recorded with a 250 or $500 \mathrm{~Hz}$ sample frequency and stored in the MUSE Cardiology Information system (GE Healthcare, Little Chalfont, UK). All analyses were performed offline using custom-made software in Matlab (2018a, Mathworks, Natick, MA, USA). To improve signal-to-noise ratio, a median complex was calculated for all individual ECG leads by aligning individual complexes on the R-peak after filtering and up-sampling to $1000 \mathrm{~Hz}$.

\section{Landmark detection}

The R-peak, QRS-onset and T-wave end were detected by our previously described algorithms 27. In short, the R-peak was detected on the root mean square ECG (ECGRMS) using the Pan-Tompkins algorithm. The onset of the QRS-complex was defined as the largest positive peak in the second derivative of the ECGRMS preceding the R-peak. The beginning of the window in which we performed our analysis was defined as R-peak+70ms. The end of the T-wave was detected by means of an automated tangent method. All ECG landmarks and median complexes were checked manually.

\section{T-wave characterization}

Hermite-Gauss polynomials (HG-polynomials) have been used to characterize the morphology of QRScomplexes ${ }^{28,29}$. A linear combination of the first two HG-polynomials were fit on the STT-segment of lead 1, II and v1 to v6. The first-order HG-polynomial is symmetrical bell-shaped whereas the second order is biphasic (Figure-1). The fitting was performed by first aligning the top of the first-order HG-polynomia and the zero crossing of the second-order HG-polynomial on the T-peak (Figure 7-1 (1)). Thereafter, linear combination of weight coefficients of the first- $\left(c_{1}\right)$ and second-order $\left(c_{2}\right) H G$-polynomial for a fixed range of HG-polynomial widths (Figure 7-1 (2)) were fit using the least-squares method ( $c_{1}$ and $c_{2^{\prime}}$ Figure 7-1 (3)). The weight coefficients of the fit that resulted in the smallest fitting error (defined as the roo mean square of the difference between the reconstructed and original signal normalized to the signal's maximum) was used as the T-wave characterization. Since a normal T-wave is positive and slightly asymmetric, $c_{1}$ will be positive and since the first-order $\mathrm{HG}$-polynomial is symmetric a small (negative) $\mathrm{C}_{2}$ is needed to capture the slightly asymmetric morphology of a normal T-wave. Abnormal morphologies will lead to abnormal weight coefficients. For example, high/low amplitude T-waves will have high/low coefficients; $c_{2}$ will be larger than $c_{1}$ in a biphasic T-wave since the second-order polynomial captures the biphasic morphology; and a negative T-wave will result in a negative c1 (Figure 7-1). Complex T-waves (e.g. notched T-waves) cannot be fit accurately with only the first- and second-order HG-polynomials. Therefore, the fitting error was also used in the characterization of the T-wave morphology. By doing so, complex T-waves that cannot be fit properly using only the first two order $\mathrm{HG}$-polynomials can still be classified as an altered T-wave because of the high fitting error.
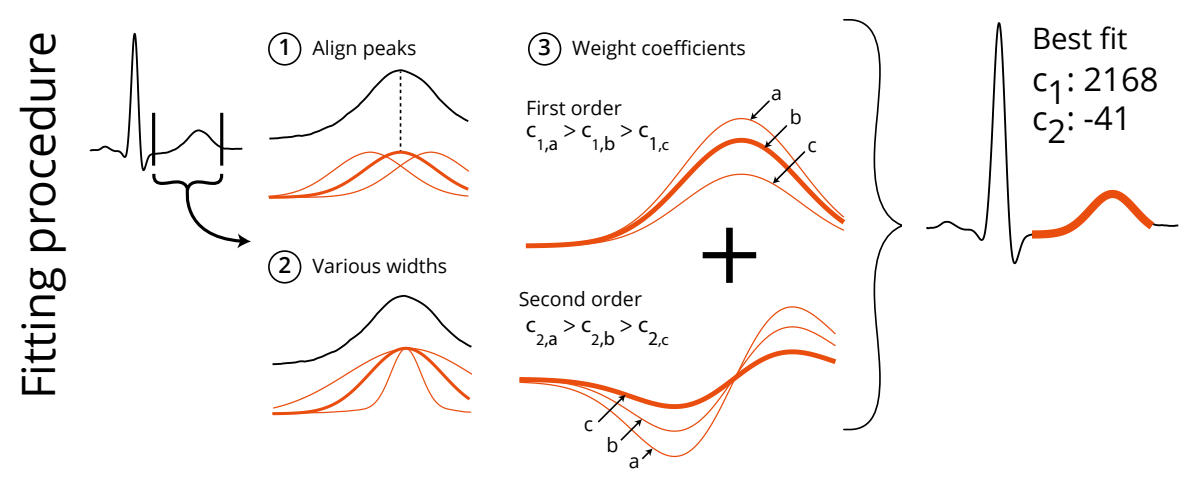

Controls

LQT-1
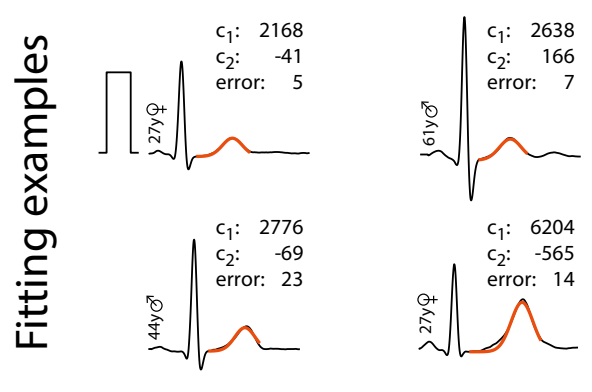

LQT-2

LQT-3

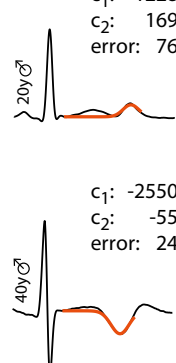

竞

$\begin{array}{lr}c_{1}:-300 \\ c_{2}: & 475 \\ \text { error: } & 45\end{array}$

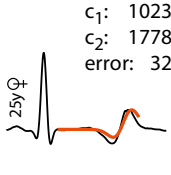

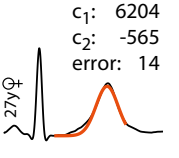

Figure 7-1 T-wave characterization using Hermite-Gauss polynomials. (1) The T-peak and the peak of the first order $\mathrm{HG}$-polynomial were aligned. (2) A fixed range of $\mathrm{HG}$-polynomial widths were fitted on the STT-segment. (3) The weight coefficients ( 1 1 and c2) obtained from the best fit were used as T-wave characterization. LQT-n, long QT-syndrome subtype n; error was defined as the root mean square of the difference between the fit and original signa.

\subsubsection{QTc cut-offs}

Two QTc cut-offs were used to classify subjects as LQTS patients or controls. First, following current HRS EHRA/APHRS Expert Consensus recommendations ${ }^{30}$, a QTc cut-off of $\geq 480 \mathrm{~ms}$ was used. Secondly, the 99th-percentile from healthy males (450ms) and females $(460 \mathrm{~ms})$ to classify LQTS patients was used as the QTc cut-off4.

\subsubsection{Machine learning}

Subjects were classified as LQTS patients or controls by three Support-Vector-Machine (SVM) models trained on three different sets of features. The first model (Baseline model) contains age, sex and QTC 
as inputs and reflects the optimal classification based on these basic and clinically used parameters. The second model (Morphology model) contains $\mathrm{C}_{1}, \mathrm{C}_{2}$ and the fitting error from the precordial ECG leads and lead I and II as well as age and sex and reflects the optimal classification based on the morphology markers without QTC. The third model (Extended model) combines all inputs from the baseline and the morphology model. A detailed description of the machine learning approach can be found in the supplementary materials. In short, all models were trained and tested on the Amsterdam data using a 10-fold cross-validation. In a 10-fold cross-validation, the entire dataset is partitioned into 10 equally sized subsets. A model is trained on 9 subsets and tested on the 10th subset. This is repeated 10 times until all subsets served once as the test set. The mean performance of these 10 validations was considered the expected performance of the final model that was trained on the entire Amsterdam data. In general, internal cross-validation is an adequate measure of external performance. However, to further investigate the robustness of our final model on new data, it was externally tested on the Leuven data. Model performances were quantified by sensitivity, specificity and the total number of correctly classified individuals (accuracy)

\subsubsection{QT-expert}

To further validate the Extended model, a QT-expert (P.P.) classified all subjects from the Leuven data as LQTS patient or control, based on the full ECG with knowledge of the subject's sex and age and the corresponding QT-, RR-intervals and QTc which were provided by our algorithms. The agreement between the Extended model and the QT-expert was evaluated.

\subsubsection{Statistical analyses}

All continuous variables are presented as mean \pm standard deviation, categorical variables are presented as number and percentage. Receiver-operating characteristic (ROC) curve analysis was used to calculate the area under the curve (AUC) as well as the sensitivity and specificity at the optimal cut-off determined by the Youden-index. DeLong's method ${ }^{31}$ was used to compare ROC curves of the Baseline, Morphology and Extended model. A P-value $<0.05$ was considered significant. All analyses were done in Matlab (2018a, Mathworks, Natick, MA, USA).

\subsection{Results}

\subsubsection{Study population}

After exclusions, 678 subjects were included in the Amsterdam data and 117 subjects were included in the Leuven data. Ten subjects were excluded from the Amsterdam data because at least one ECG ead contained too much noise. The final Amsterdam data consisted of 345 genotype-negative family members and 333 LQTS patients (126 LQT-1, 156 LQT-2, 51 LQT-3). No subjects were excluded from the Leuven data which consisted of 45 genotype-negative family members and 72 LQTS patients (16 LQT-1, 51 LQT-2, 5 LQT-3). Study population characteristics for both datasets are shown in Table 7-1.

\begin{tabular}{|l|lll|lll|}
\hline \multicolumn{3}{|c}{ Amsterdam } \\
\hline & Age (years) & Sex (m/f) & QTc (ms) & Age (years) & Sex (m/f) & QTc (ms) \\
Controls & $45 \pm 15$ & $160 / 185$ & $410 \pm 28$ & $42.8 \pm 16.6$ & $18 / 27$ & $402 \pm 27$ \\
LQTS & $42 \pm 15$ & $137 / 196$ & $457 \pm 38$ & $37.5 \pm 14.0$ & $32 / 40$ & $456 \pm 37$ \\
LQT-1 & $41 \pm 15$ & $49 / 77$ & $455 \pm 34$ & $44.3 \pm 9.4$ & $4 / 12$ & $467 \pm 44$ \\
LQT-2 & $42 \pm 15$ & $69 / 87$ & $462 \pm 36$ & $35.7 \pm 15.0$ & $28 / 23$ & $455 \pm 34$ \\
LQT-3 & $40 \pm 15$ & $19 / 32$ & $446 \pm 50$ & $34.8 \pm 10.2$ & $0 / 5$ & $421 \pm 11$ \\
\hline
\end{tabular}

Table 7-1 Study population characteristics. Values are expressed as mean \pm standard deviation. $m$ male; f, female; QTc, QTc Bazett; ms, milliseconds,

\subsubsection{Classification results}

A large overlap in QTC between patients and controls exists in both the Amsterdam (top left) and the Leuven data (top right) as illustrated in Figure 7-2. Subjects incorrectly classified by our Extended model are shown as dark blue dots. The majority of subjects (84\%) are classified correctly by the Extended model. Moreover, the Extended model was able to correctly classify LQTS patients with a QTC as low as $400 \mathrm{~ms}$, while it was also capable of correctly classifying controls with a QTc up to $460 \mathrm{~ms}$. Almos all incorrectly classified patients are LQTS patients with a normal QTc while the majority of incorrectly classified controls have a prolonged QTc. The ten parameters with the highest weight (most important)

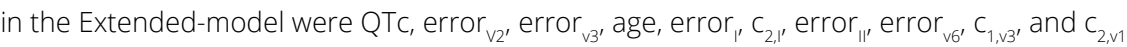

Accuracies of respectively the Amsterdam and Leuven data for the Baseline (80\% and 76\%), Morphology (76\% and 1\%) and Extended model (84\% and 79\%) as well as of the two clinically used QTc cut-offs (62\% and 52\% for QTc $\geq 480 \mathrm{~ms}$ and $72 \%$ and $65 \%$ for QTc $\geq 450 / 460 \mathrm{~ms}$ ) are shown in the lower half of Figure 7-2. The chart shows that the Extended model has the best performance while the QTc cut-offs have the poorest performance in both datasets. It can furthermore be noted that the performance of the different classification methods used are similar between both datasets, indicating that the mode is robust. The accuracy was furthermore determined on a subset of the data in which all overt LQTS subjects (QTc>450/460ms) were excluded. The Extended model had the highest performance (80.8\%) whereas the Baseline model had the lowest accuracy (75.7\%).

Sensitivity and specificity for each model or QTc cut-off are shown in Table 7-2. The best sensitivity for both datasets was achieved with the Extended model (83\% and 75\%) followed by the Baseline model (75\% and 69\%). As expected from the QTc distributions, using only QTc cut-off to diagnose LQTS results in the highest specificity ( $99 \%$ and $98 \%$ ) with the lowest sensitivity for both datasets (24\% and $24 \%$ respectively). No AUC and confidence intervals were calculated for the Leuven data since the models and cut-offs obtained from the Amsterdam data were applied on the Leuven data and therefore no ROC curve was generated for the Leuven data.

The AUC of the Extended model was significantly higher compared to both Baseline and Morphology model $(p<0.001)$ using Delong's method to compare ROCs. The AUC of the Baseline model furthermore was significantly higher compared to the Morphology model $(p<0.001)$ 


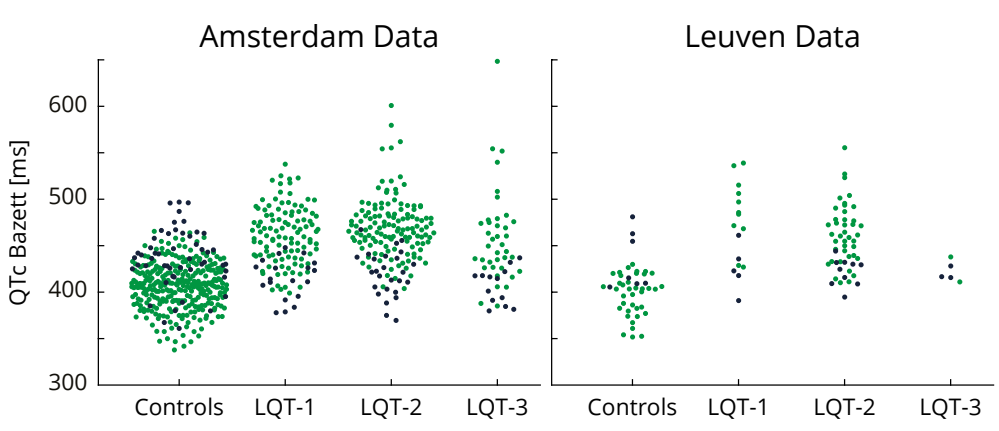

- Correctly classified
- Incorrectly classified

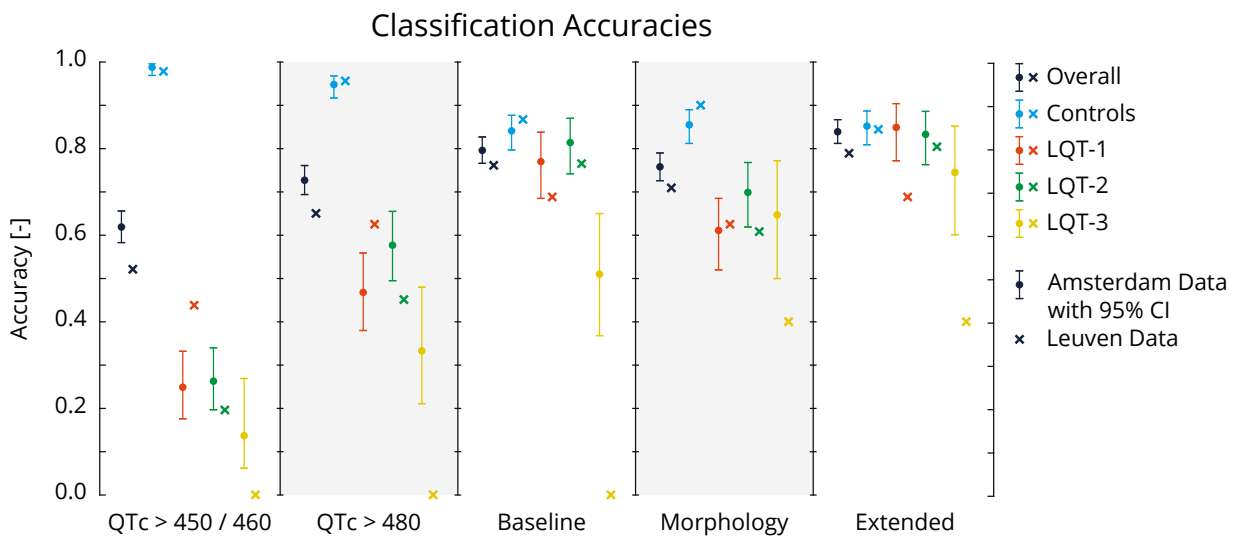

Figure 7-2 Classification accuracies of the various models on the Amsterdam and Leuven dataset. QTc, QTc Bazett; $m$, male; f, female; ms, milliseconds; LQT-n, long-QT syndrome subtype $n$.

\begin{tabular}{|c|c|c|c|c|c|}
\hline & \multicolumn{3}{|c|}{ Amsterdam } & \multicolumn{2}{|c|}{ Leuven } \\
\hline & Sens.[\%] & Spec.[\%] & AUC & Sens.[\%] & Spec.[\%] \\
\hline $\mathrm{QTC}>480$ & $\begin{array}{l}24 \\
(19-29)\end{array}$ & $\begin{array}{l}99 \\
(97-100)\end{array}$ & - & 24 & 98 \\
\hline QTc $>450 / 460$ & $\begin{array}{l}50 \\
(44-55)\end{array}$ & $\begin{array}{l}95 \\
(92-97)\end{array}$ & - & 46 & 96 \\
\hline Baseline & $\begin{array}{l}75 \\
(70-80)\end{array}$ & $\begin{array}{l}84 \\
(80-88)\end{array}$ & $\begin{array}{l}0.87 \\
(0.84-0.90)\end{array}$ & 69 & 87 \\
\hline Morphology & $\begin{array}{l}66 \\
(60-71)\end{array}$ & $\begin{array}{l}86 \\
(81-89)\end{array}$ & $\begin{array}{l}0.81 \\
(0.77-0.84)\end{array}$ & 60 & 89 \\
\hline Extended & $\begin{array}{l}83 \\
(78-86)\end{array}$ & $\begin{array}{l}85 \\
(81-89)\end{array}$ & $\begin{array}{l}0.90 \\
(0.88-0.93)\end{array}$ & 75 & 84 \\
\hline
\end{tabular}

Table 7-2 ROC analyses of the various models on the Amsterdam and Leuven datasets. Sens, sensitivity; spec, specificity; AUC, area under the curve; QTc, QT corrected for heart rate using Bazett's formula.

\subsubsection{LQTS subtypes}

The accuracy for LQTS subtypes and controls is furthermore shown in Figure 7-2. It can be appreciated that the Extended model has the highest accuracy for all LQTS patients but especially in LQT-3 patients. QTc $\geq 480 \mathrm{~ms}$ has the highest accuracy in controls but the lowest accuracy in LQTS patients.

\subsubsection{QT-expert}

The QT-expert had a total accuracy of $79 \%(n=93)$ on the Leuven data with a sensitivity and specificity of 75\% and 86\% respectively (see Table 7-3). The Extended model and the QT-expert agreed in 87\% ( $n=102$ ) of all cases. In 17 subjects (13 LQTS, 4 controls) both the QT-expert and the Extended mode were wrong. The QT-expert indicated that he was not sure about his diagnosis in $24 \%$ of all cases $(n=28)$.

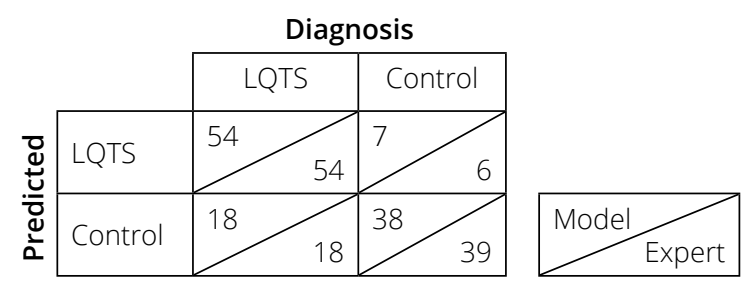

Table 7-3 Number of (in)correctly classified subjects by the Extended model and the QT-expert. QTS, long-QT syndrome.

\subsection{Discussion}

In this study, we evaluated the additional value for diagnosing LQTS of the T-wave morphology characterized by HG-polynomials with respect to various diagnostic SVM-models/thresholds based on QTC. The Extended model has the best accuracy on both the Amsterdam (84\%) and the Leuven data (79\%) closely followed by the Baseline model (80\% and $76 \%$ respectively). In contrast to the Baseline model, the Extended model is even able to accurately diagnose LQTS patients with a QTC down to $400 \mathrm{~ms}$. ROC curve comparison by Delong's method showed that the AUC of the Extended model was significantly higher compared to both the Baseline as well as the Morphology model. The predictions by the Extended model moreover has a good agreement with the QT-expert. This indicates that the Extended model cannot replace QT-experts but can be used by physicians less familiar/experienced with diagnosing LQTS. We are willing to share our Matlab-code on request by e-mail. Future users should however realise that the model was not trained and tested on other pathologies that affect the STTsegment like ischemia, ion-disturbances or ventricular conduction problems.

When looking at the accuracy per LQTS subtype, it can be seen that although the Baseline model's performance in controls, LQT-1 and LQT-2 patients, does not differ that much from the Extended model, the Extended model as well as the Morphology model do increase the sensitivity for LQT-3 patients. An improved diagnosis for LQT-3 patients itself is relevant since cardiac events are reported to be letha more frequently in LQT-3 patients ${ }^{32}$. Also, it is important to note that since the LQT-3 patients account for only $7 \%$ and $4 \%$ of all subjects in the Amsterdam and Leuven data respectively, this improved sensitivity is hardly reflected by the total number of correctly classified subjects. The low number of LQT-3 patients 
is indeed a limitation of this study and more LQT-3 patients are needed to investigate whether this observation is true.

Strikingly, the QTC>480ms cut-off, recommended by the current Expert Consensus, has the poores performance with an accuracy of $62 \%$ and $52 \%$ on the Amsterdam and Leuven data respectively. Based on these guidelines, in subjects with a QTc $<480 \mathrm{~ms}$, LQTS can only be diagnosed with a Schwartzscore $\geq 3.5$ or an unequivocally pathogenic mutation in one of the LQTS genes ${ }^{2,30}$. It has however been shown that the Schwartz-score>3 has a high specificity (99\%) but a low sensitivity (36\%) in diagnosing disease carriers ${ }^{11}$. Of note, subjects with a QT $<480 \mathrm{~ms}$ might never reach a Schwartz-score of $\geq 3.5$ since Torsade de pointes, T-wave alternans, notched T-waves or a clinical history with syncope is needed to reach 3.5 but are found very infrequently and/or are not specifici, 1 .

Other studies also showed poor predictive performance of the $\geq 480 \mathrm{~ms}$ QTC cut-off with similar sensitivity and specificity (26\% and $99 \%$ respectively) $)^{4}$ or recommend lower cut-offs ${ }^{11}$. Although lower cut-offs will lead to a higher sensitivity, they will be accompanied by a decrease in specificity. The wellknown considerable overlap4,6 in QTc between patients and controls will hamper LQTS diagnosis no matter which fixed QTc cut-off is used. Our Baseline model shows that LQTS diagnosis can already be improved by using a sex- and age-dependent QTc cut-off. The advantage of the Baseline model is that it can be used without sophisticated software. As shown by the improved accuracy of the Extended mode and the Morphology model for LQT-3 patients, introducing T-wave morphology markers in the diagnosis of LQTS further solves the issue of the considerable overlap in QTc between patients and controls.

The morphology score developed by Andersen et al..$^{33}$ is frequently used to characterize the T-wave morphology and has been shown to improve LQTS diagnosis 25,26 . One of its strengths is that the interpretation of the parameters is easy to understand and can guide cardiologists in the manual assessment of the T-wave morphology. A downside of the score is that it only describes altered LQTS T-wave morphology characteristics known so far. Unrecognized altered LQTS T-wave morphology characteristics might therefore not lead to an abnormal morphology score. In this study we therefore developed an objective method in which the SVM-model decides which characteristics are important. This makes our HG-polynomial based method unbiased by current knowledge. Sugrue et al. developed a T-wave analysis program that is also not based on known LQTS T-wave morphology characteristics ${ }^{34}$ Although the overall accuracy of their T-wave analysis program is comparable with ours (86\%), it should be noted that Sugrue et al. included age- and sex-matched controls with no history of cardiac diseases. A head-to-head comparison between both algorithms should assess whether either algorithm has improved accuracy over the other, or whether there are certain subgroups in which one performs better. Combining T-wave morphology characterizations of Sugrue et al. with ours might even lead to a new, improved model.

In this study we only used the first two orders of the HG-polynomials to reduce the number of features used in our approach. The errors of the fit in lead I, II, v2, v3 and v6 were among the most important parameters in the Extended model. This indicates that the complexity of the T-wave is an important classification in the diagnosis of LQTS. Although adding more orders or fitting more parameters might improve the fits and the diagnosis, more data is likely needed for these more complicated models and parameters. Unfortunately, current datasets do not include follow-up ECGs. Therefore, the reproducibility of our algorithm could not be addressed.
The aim of this study was to improve the diagnosis of genotype-positive LQTS patients. DNA testing was used as the gold standard for diagnosing LQTS. However, DNA testing might not (yet) be a proper gold standard for diagnosing LQTS since it has been shown that approximately $20 \%$ of clinically diagnosed LQTS patients remain genetically elusive ${ }^{8,9}$. Vice versa, pathogenic variants seem to have a reduced penetrance and therefore do not always lead to symptoms and/or a prolonged QT-interval,a. Predicting phenotype-positive LQTS patients based on symptomaticity might therefore be more usefu than predicting genotype-positive LQTS patients. However, studying symptomaticity bears other clinical challenges since patients should ideally be off-treatment and followed intensively. Therefore, DNA testing is still the most objective diagnostic method currently available.

\subsection{Conclusion}

In this study we showed that T-wave morphology can be characterized by using an automatic algorithm that fits the T-wave using the first two Hermite-Gauss polynomials. We furthermore showed that support-vector-machine model based on combining weight coefficients of Hermite-Gauss polynomials and the error of the fit, with age, gender and QTC significantly improves the diagnosis of genotypepositive LQTS patients and their genotype-negative family members based on standard ECGs. 


\subsection{References}

1. Priori SG, Wilde AA, Horie M, et al.: HRS/EHRA/APHRS Expert Consensus Statement on the Diagnosis and Management of Patients with Inherited Primary Arrhythmia Syndromes. Heart Rhythm Elsevier 2013;10:1932-1963.

2. Schwartz PJ, Moss AJ, Vincent GM, Crampton RS: Diagnostic criteria for the long QT syndrome. An update. Circulation 1993;88:782-784.

3. Bazett HC: An analysis of the time-relation of electrocardiograms. Heart 1920;7:353-370.

4. Vink AS, Neumann B, Lieve KW, et al.: Determination and Interpretation of the QT Interval. Circulation 2018;138:2345-2358.

5. Viskin S, Rosovski U, Sands Al, et al.: Inaccurate electrocardiographic interpretation of long QT: The majority of physicians cannot recognize a long QT when they see one. Heart Rhythm 2005;2:569574.

6. Viskin S: The QT interval: Too long, too short or just right. Heart Rhythm Heart Rhythm Society, 2009;6:711-715.

7. Vink AS, Clur SAB, Wilde AAM, Blom NA: Effect of age and gender on the QTC-interval in healthy individuals and patients with long-QT syndrome. Trends Cardiovasc Med Elsevier, 2018:28:64-75.

8. Kapplinger JD, Tester DJ, Salisbury BA, et al.: Spectrum and prevalence of mutations from the firs 2,500 consecutive unrelated patients referred for the FAMILION® long QT syndrome genetic test. Heart Rhythm Elsevier Inc., 2009;6:1297-1303.

9. Crotti L, Spazzolini C, Schwartz PJ, et al.: The common long-QT syndrome mutation KCNQ1/A341V causes unusually severe clinical manifestations in patients with different ethnic backgrounds: Toward a mutation-specific risk stratification. Circulation 2007;116:2366-2375.

10. Giudicessi JR, Roden DM, Wilde AAM, Ackerman MJ: Classification and Reporting of Potentially Proarrhythmic Common Genetic Variation in Long QT Syndrome Genetic Testing. Circulation 2018;137:619-630

11. Hofman N, Wilde AAM, Kaab S, et al.: Diagnostic criteria for congenital long QT syndrome in the era of molecular genetics: do we need a scoring system? Eur Heart J 2007;28:575-580.

12. Robyns $T$, Willems $R$, Vandenberk $B$, et al.: Individualized corrected $Q T$ interval is superior to $Q T$ interval corrected using the Bazett formula in predicting mutation carriage in families with long QT syndrome. Heart Rhythm Elsevier Inc., 2017;14:376-382.

13. Vyas H, Hejlik J, Ackerman MJ: Epinephrine QT Stress Testing in the Evaluation of Congenital LongQT Syndrome. Circulation 2006;113:1385-1392.

14. Shimizu W, Noda T, Takaki H, et al.: Diagnostic value of epinephrine test for genotyping LQT1, LQT2, and LQT3 forms of congenital long QT syndrome. Heart Rhythm 2004;1:276-283.

15. Sy RW, Van Der Werf C, Chattha IS, et al.: Derivation and validation of a simple exercise-based algorithm for prediction of genetic testing in relatives of LQTS probands. Circulation 2011;124:21872194.

16. Horner JM, Horner MM, Ackerman MJ: The diagnostic utility of recovery phase QTC during treadmill exercise stress testing in the evaluation of long QT syndrome. Heart Rhythm Elsevier Inc, 2011;8:1698-1704.

17. Viskin S, Postema PG, Bhuiyan ZA, et al.: The Response of the QT Interval to the Brief Tachycardia Provoked by Standing. I Am Coll Cardiol Elsevier Inc., 2010;55:1955-1961.

18. Adler A, van der Werf C, Postema PG, et al.: The phenomenon of "QT stunning": The abnormal QT prolongation provoked by standing persists even as the heart rate returns to normal in patients with long QT syndrome. Heart Rhythm Elsevier Inc., 2012;9:901-908.

19. Chorin E, Havakuk $O$, Adler $A$, et al.: Diagnostic value of T-wave morphology changes during "OT stretching" in patients with long QT syndrome. Heart Rhythm Elsevier, 2015;12:2263-2271.

20. Lehmann MH, Suzuki F, Fromm BS, et al.: T wave "humps" as a potential electrocardiographic marker of the long QT syndrome. J Am Coll Cardiol Elsevier Masson SAS, 1994;24:746-754.

21. Moss AJ, Zareba W, Benhorin J, et al.: ECG T-Wave Patterns in Genetically Distinct Forms of the Hereditary Long QT Syndrome. Circulation 1995;92:2929-2934.

22. Yan G-X, Antzelevitch C: Cellular basis for the normal T wave and the ECG manifestations of the long QT syndrome. J Electrocardiol 1998;30:148.

23. Schwartz PJ: Clinical significance of QT prolongation: a personal view. In Butrous G, Schwartz PJ, eds: Clinical Aspects of Ventricular Repolarization. London: Farrand Press, 1989, pp. 343-356.

24. Schwartz PJ, Ackerman M]: The long QT syndrome: A transatlantic clinical approach to diagnosis and therapy. Eur Heart J 2013;34:3109-3116.

25. Hermans BJM, Stoks J, Bennis FC, et al.: Support vector machine-based assessment of the T-wave morphology improves long QT syndrome diagnosis. EP Eur 2018;20:iii113-iii119.

26. Porta-Sánchez A, Spillane DR, Harris L, et al.: T-Wave Morphology Analysis in Congenital Long QT Syndrome Discriminates Patients From Healthy Individuals. JACC Clin Electrophysiol 2017;3:374381

27. Hermans BJM, Vink AS, Bennis FC, et al.: The development and validation of an easy to use automatic QT-interval algorithm. Baumert M, ed: PLoS One 2017:12:e0184352.

28. Laguna $\mathrm{P}$, Jané $\mathrm{R}$, Olmos $\mathrm{S}$, et al.: Adaptive estimation of QRS complex wave features of ECG signal by the Hermite model. Med Biol Eng Comput 1996;34:58-68.

29. Lyon A, Ariga R, MincholéA, et al.: DistinctECG Phenotypes Identified in Hypertrophic Cardiomyopathy Using Machine Learning Associate With Arrhythmic Risk Markers. Front Physiol 2018;9:1-13.

30. Priori SG, Wilde AA, Horie M, et al.: HRS/EHRA/APHRS Expert Consensus Statement on the Diagnosis and Management of Patients with Inherited Primary Arrhythmia Syndromes: Document endorsed by HRS, EHRA, and APHRS in May 2013 and by ACCF, AHA, PACES, and AEPC in June 2013. Hear Rhythm Elsevier, 2013;10:1932-1963.

31. DeLong ER, DeLong DM, Clarke-Pearson DL: Comparing the Areas under Two or More Correlated Receiver Operating Characteristic Curves: A Nonparametric Approach. Biometrics 1988:44:837.

32. Zareba W, Moss AJ, Schwartz PJ, et al.: Influence of the Genotype on the Clinical Course of the LongQT Syndrome. N Engl J Med 1998;339:960-965.

33. Andersen MP, Xue JQ, Graff C, et al.: A robust method for quantification of IKr-related T-wave morphology abnormalities. 2007 Comput Cardiol IEEE, 2007, pp. 341-344.

34. Sugrue A, Noseworthy PA, Kremen V, et al.: Identification of Concealed and Manifest Long Q Syndrome Using a Novel T Wave Analysis Program. Circ Arrhythmia Electrophysiol 2016;9:1-8. 


\subsection{Supplementary materials}

\subsubsection{Machine learning methods}

The total Amsterdam dataset was divided into 10 folds of equal size with an equal ratio of LQTS patients to controls within each fold. Each fold served as a test set once, while the remaining nine folds served as the training set. Within each training fold, all features were normalized to have a mean of zero and a variance of one. The test set was normalized using the original mean and variance of the features of the training set to avoid any influence of the test set. For every fold, support-vector-machine (SVM) models with 30 configurations consisting of every possible combination of three kernels (linear, radia and 2nd order polynomial kernel) and ten different box constraints were trained and tested. The box constraint parameter (varied from $1 \mathrm{e}^{-5}$ to $1 \mathrm{e}^{5}$ in multiplications of 10) indicates how strictly the model separates between LQTS and controls, with higher box constraint values leading to a stricter separation. The different kernels use different functions to transfer the originally possibly non-linear data into a higher-dimensional space in which the data becomes separable. All 30 model configuration calculated a score reflecting the probability of exhibiting LQTS for all subjects once. Receiver operator characteristics curves were calculated based on these scores and the model configuration that resulted in the highest area under the curve was considered as the optimal SVM model configuration. Since the composition of the ten folds can have an effect on the optimal model configuration, the optimization was repeated 100 times whilst varying the division of subjects over the folds. The model configuration that had the highest area under the curve the most frequently was used as the optimal configuration and was trained and tested using a final 10-fold cross-validation. All steps were repeated for the three different feature sets (Baseline, Morphology and Extended model) and only the performances of the optimized SVM model were reported for every feature set. Finally, one model was trained on the entire dataset using the optimal configuration for the feature set. This final model was thereafter validated on the external validation set. 


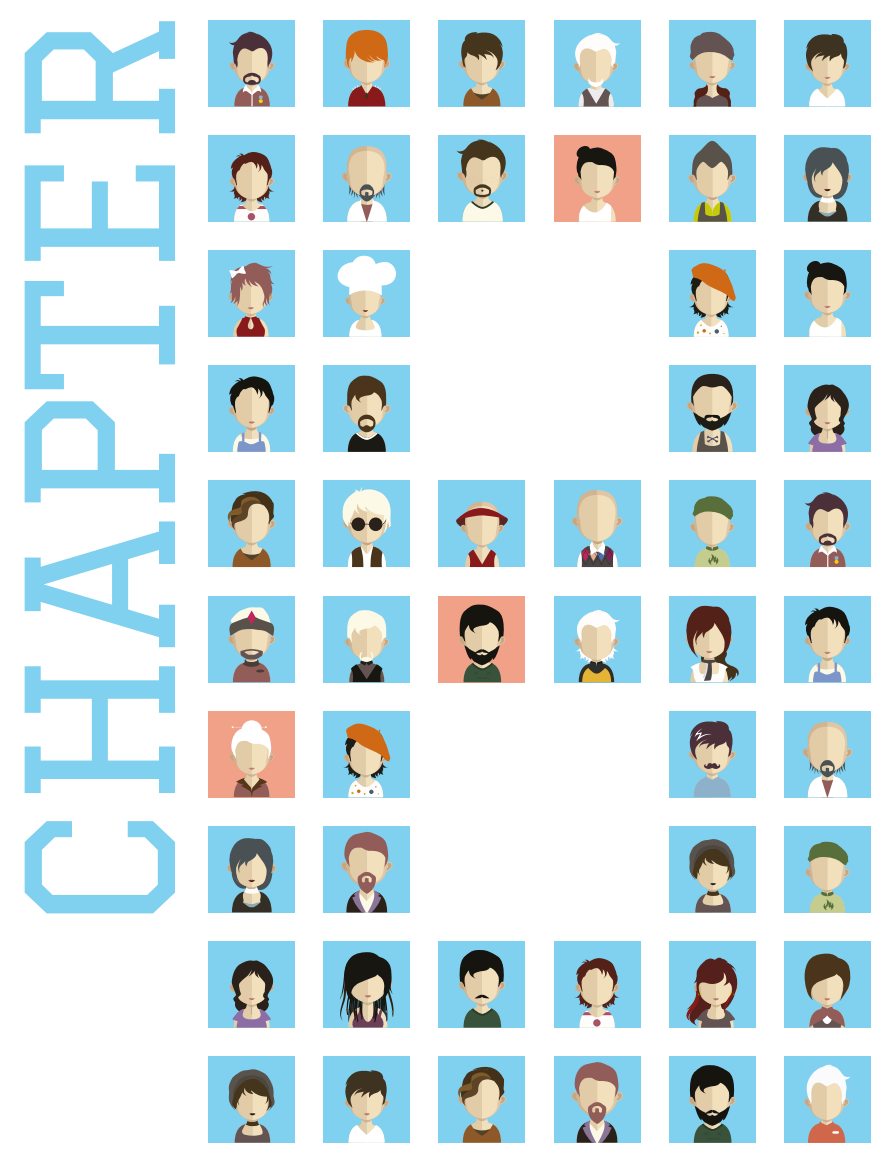

\section{CHAPTER 8}

THE IMPACT OF PULMONARY VEIN ISOLATION ON THE QT-INTERVAL 
Abstract

\section{Background}

Pulmonary vein isolation (PVI) has become the cornerstone treatment for atrial fibrillation (AF). Unintentional modulation of the ganglionated plexi by PVI was suggested to possibly affect ventricular electrophysiology.

Objective

The objective of this study is to examine whether standard of care PVI induces a change in the relationship between heart rate and ventricular repolarization, as indicated by the QT-interval corrected for heart rate (QTC)

Methods

Data was gathered from a registry of 279 AF patients scheduled for PVI. Five-minute-long electrocardiograms were recorded at hospital's admission $\left(T_{10}\right)$, one day after the procedure $\left(T_{\text {t1 }}\right)$ and at three months post-procedure $\left(T_{+3 m}\right)$. Only patients who were in sinus rhythm at all three recordings ( $n=117,42 \%$ ) were included in the study. QT intervals were with custom-made software and QTC was calculated using Fridericia's formula. A repeated-measures ANOVA with Bonferron post-hoc correction was used to compare intervals obtained at the three different recording times.

Results

The QT- and RR-intervals were significantly shortest at $\mathrm{T}_{+1 \mathrm{~d}}$ and longest at $\mathrm{T}_{-1 \mathrm{~d}^{\circ}}$. There was no statistically significant within-subject difference in QTc between the recordings. Results from a univariate analysis of variance showed that age $(p=0.06)$, sex $(p=0.81)$, self/non-self-terminating $A F(p=0.31$, blation technique $(p=0.08)$, first or re-do procedure $(p=0.76)$ and self-reported AF recurrence at 12 months post-PVI $(p=0.72)$ did not influence the QTc-interval at $\mathrm{T}_{+3 \mathrm{~m}}$ with respect to the QTc at $\mathrm{T}_{-1 \mathrm{~d}}$

Conclusion

In this study we showed that standard of care PVI with possible moderation of the ganglionated plexi, does not induce a change in the relationship heart rate and ventricular repolarization.

\subsection{Introduction}

An impaired parasympathetic activity (or increased sympathetic activity) is known to increase the susceptibility of the heart to ventricular arrhythmias $(\mathrm{VA})^{1,2}$. Since parasympathetic postsynaptic neurons are mainly located in the atrial ganglionated plexi (GP) $)^{3-5}$, modulation of the GP may alter ventricular autonomic innervation. Atrial GP located close to the pulmonary veins may be modulated by pulmonary vein isolation (PVI), the cornerstone treatment of atrial fibrillation (AF) $)^{6}$. Unintentional partial atria denervation caused by modulation of the GP is known to increase the heart rate 7.8 but might also affect ventricular electrophysiology ${ }^{9-13}$. It has been shown that a small number of AF patients develop outflow tract ventricular premature beats (VPB) and non-sustained ventricular tachycardias after isolation of the pulmonary veins $\mathrm{S}^{10,12}$. This might be caused by an affected ventricular electrophysiology as a consequence of altered autonomic innervation

Recently, an experimental study in murine hearts showed that mechanical disruption or pharmacological blockade of parasympathetic innervation shortened ventricular effective refractory perios (ERP) and increased susceptibility to VA with programmed stimulation. Another experimental study in can hearts also showed an increased susceptibility to VA after a targeted GP ablation but contrary to the previous findings reported a significantly increased ventricular ERP'13. It was furthermore shown that the ventricular action potential duration (APD) significantly increased in canine hearts after ablating the GP13.

To the best of our knowledge, it is unknown whether the APD increases in AF patients after PVI. This can be relevant to know since an increased APD is associated with $V A^{14,15}$ and might therefore explain new onset VA in post-PVI patients. In normal physiological circumstances, the APD adapts to changes in hear rate ${ }^{16}$. Since the heart rate often increases in post-PVI AF patients (because of an inevitably modulation of the intracardiac neural network ${ }^{7-9}$ ) the capability of the (ventricular) APD to adapt to changes in heart rate might also be disturbed because of the neural modulations. An adaptation of the APD is especially relevant in patients with AF recurrence because of the irregular ventricular rates. The objective of this study therefore is to examine whether a PVI induces a mismatch between heart rate and APD. In this clinical study we measure the QT-interval as a surrogate marker for gross ventricular APD and assume that a change in the relationship between gross ventricular APD and heart rate should be reflected by a change in the QT-interval corrected for heart rate (QTC).

\subsection{Methods}

\subsubsection{Study population}

All data used in this study were gathered from a registry of AF patients scheduled for PVI at the Maastricht University Medical Centre. Inclusion criteria of the registry were: documented AF; $\geq 18$ years of age; scheduled for an AF ablation and able and willing to provide informed consent. Since in this study we focus on the QT-interval, we excluded all pathologies that possibly influence the QT-interval (e.g. left/right bundle branch block, ventricular paced rhythms, etc.). We only included patients who were in sinus rhythm during the ECG since an irregular ventricular rhythm might also affect the QT-interval. Written informed consent was obtained from every patient and the study protocol was approved by the hospital's ethics committee. 


\subsubsection{Ablation procedure}

All patients included in the registry received a standard AF ablation procedure following institutional's protocols. The technique used for PVI was either a wide antral circular ablation using radiofrequency (RF) energy, a cryoablation using the cryoballoon or a hybrid procedure. The decision which technique was performed was at the treating physician's discretion and was based on medical indication and the physician's preference. Since all patients only received a PVI and no mapping of AF was performed, antiarrhythmic drugs were continued throughout the study.

\subsubsection{ECG recordings and analysis}

As part of the registry, patients received a five-minute long 12-lead ECG recording at hospital's admission $\left(T_{-1 d}\right)$, one day after the procedure $\left(T_{+10}\right)$ and at the first routine outpatient clinic visit at three months post-procedure $\left(T_{+3 m}\right)$. Only patients who were in sinus rhythm during all three ECG recordings were included in the study. Hospital's admission typically was on the day or morning before the procedure. The ECG at $T$, was performed on the evening or morning after the procedure. ECGs were recorded for five minutes using the Cam-USB (GE Healthcare, Piscataway, NJ, USA) or YRS100 ECG-system (YourRhythmics, Maastricht, The Netherlands) with a $500 \mathrm{~Hz}$ sample frequency and stored for offline analysis using Matlab 2018a (Mathworks, Natick, MA, USA).

To improve signal-to-noise ratio, a median complex was constructed for all individual ECG leads from aligned individual complexes on the R-peak after filtering and up-sampling to $1000 \mathrm{~Hz}$. The R-peak, QRS-onset and T-wave end were detected using our previously described algorithms ${ }^{17}$. In short, the R-peak was detected on the root mean square ECG (ECGRMS) using the Pan-Tompkins algorithm ${ }^{18}$. The onset of the QRS-complex was defined as the largest positive peak in the second derivative of the ECGRMS preceding the R-peak. The end of the T-wave was detected by means of an automated tangent method $^{17}$. All landmarks and median complexes were checked manually and edited or deleted when necessary. The QT-interval was corrected for heart rate (QTc) using Fridericia's formula (QTc = QT / RR 1/3) since studies have shown that Fridericia's correction is the most accurate heart rate correction 19,20. QT dispersion over all twelve leads was calculated as the difference between the latest and earliest T-wave end between ECG leads of the median complex. AF recurrence was measured using a questionnaire at 12 months post-PVI.

\subsubsection{Statistical methods}

All continuous variables are presented as median and interquartile range and categorical variables are presented as frequencies and percentages. QTc, QT- and RR-intervals obtained at the three different ECG recording times were compared using repeated-measures ANOVA with Bonferroni post-hoc correction for comparison between means. The influence of age, sex, self/non-self-terminating AF, ablation technique, first or re-do procedure and self-reported AF recurrence at 12 months post-PVI on the changes in QTc were studied using a univariate analysis of variance. A p-value of $<0.05$ was considered statistically significant.

\subsection{Results}

\subsubsection{Study population}

Out of 279 patients included in the registry, $117(42 \%)$ met the inclusion criteria and were included in this study. Their mean age was $64 \pm 8$ years, $48(41 \%)$ were females and $76(65 \%)$ had self-terminating AF while the other 41 (35\%) had non-self-terminating AF. The majority of patients $(n=78 ; 67 \%)$ were treated using the cryoballoon. The remaining patients were treated with RF ( $n=34 ; 29 \%)$ or were treated with a Hybrid approach ( $n=5 ; 4 \%$ ). The majority of this cohort were re-do patients $(n=94 ; 80 \%)$. The response rate of the questionnaire was $71 \%(n=83)$. The recurrence rate of the 83 patients who did response to the questionnaire was $53 \%$

\subsubsection{Influence of PVI on QT-intervals}

Figure 8-1 shows the absolute QTc, QT- and RR-intervals (left) as well as their change relative to T-1d (right). There was a statistically significant within-subjects difference in QT and RR between the three recording times determined by a repeated measures ANOVA $(p<0.001)$.

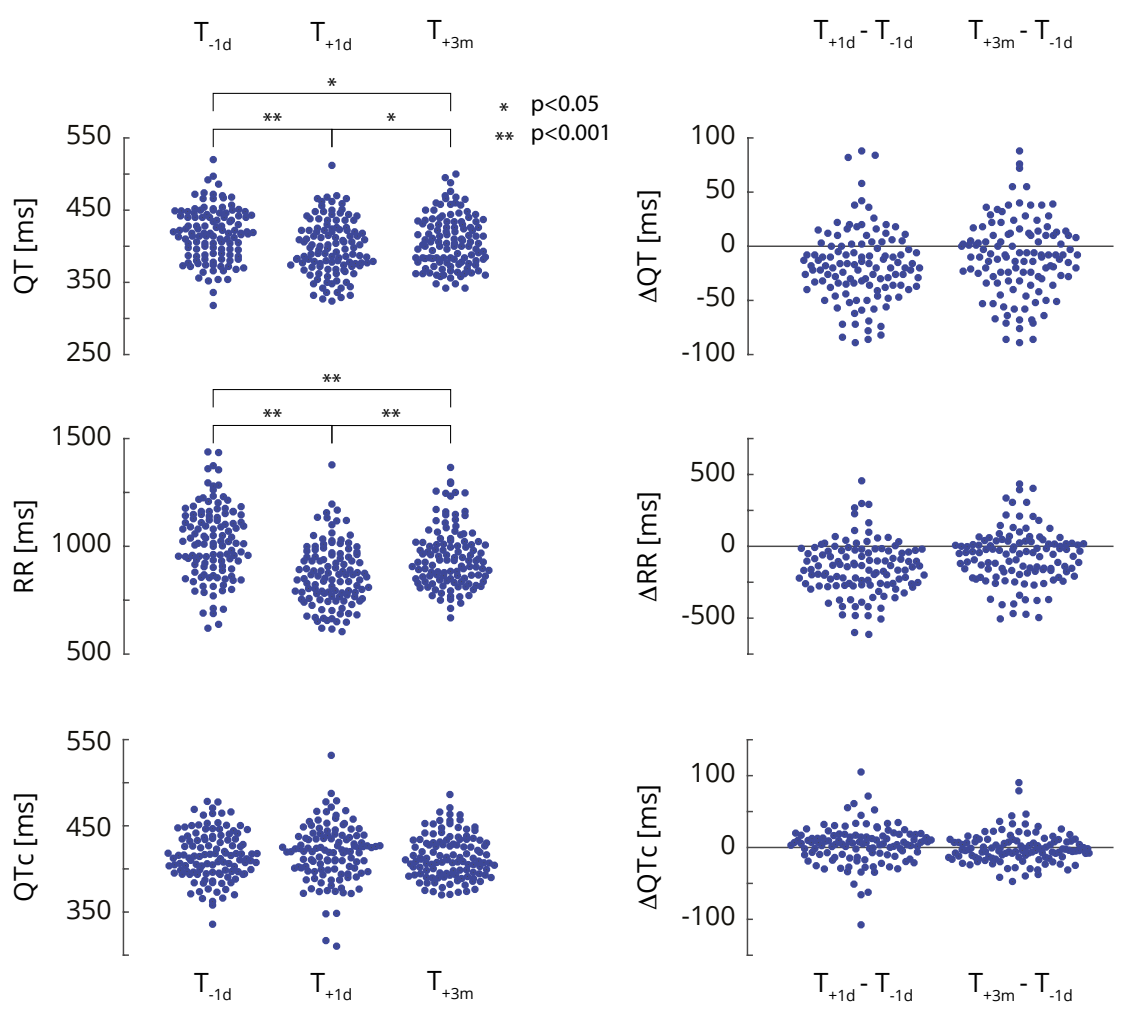

Figure 8-1 QTC, QT-, RR-intervals before and after pulmonary vein isolation. QTc, QT corrected for heart rate using Fridericia's formula; $T_{-10^{\prime}}$ one day prior to the procedure; $T_{+10^{\prime}}$ one day post procedure; $\mathrm{T}_{+3 m^{\prime}} 3$ months post procedure. 
The results of the Bonferroni post hoc tests, shown in Table 8-1, show that the QT- and RR-intervals were shortest at $T$ and longest at $T$. There was no statistically significant within-subject difference in QTc between the recordings. Also, the Bonferroni post hoc tests for QTc did not show any significant differences.

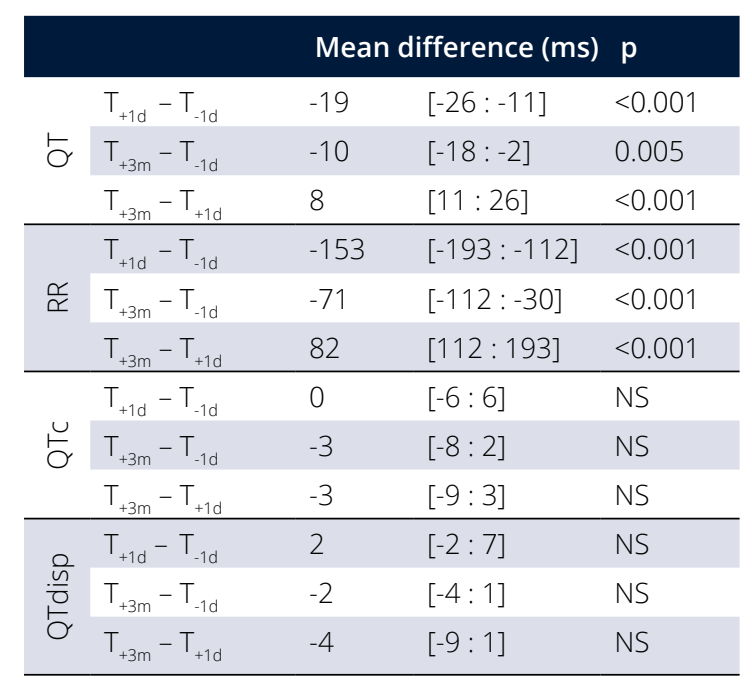

Table 8-1 Bonferroni post hoc test results. Mean differences are presented with 95\% confidence intervals between brackets. $T_{-1 d^{\prime}}$ one day prior to the procedure; $T_{+1 d^{\prime}}$ one day post procedure; $T_{+3 m^{\prime}} 3$ months post procedure; NS, not significant.

Results from the univariate analysis of variance showed that age $(p=0.06)$, sex $(p=0.81)$, self/non-selfterminating $A F(p=0.31)$, ablation technique $(p=0.08)$, first or re-do procedure $(p=0.76)$ and self-reported Af recurrence at 12 months post-PVI $(p=0.72)$ did not influence $Q T c$ at $T_{+3 m}$ with respect to the $Q T$ Tat $T_{-1 d}$

Figure 8-2 shows the QT dispersion at $\mathrm{T}_{-1 \mathrm{~d}^{\prime}} \mathrm{T}_{+1 \mathrm{~d}^{\prime}}$ and $\mathrm{T}_{+3 \mathrm{~m}}$ (left) as well as the QT dispersion change with respect to $T$. There was no statistically significant difference in QT dispersion determined by the repeated-measures ANOVA as well as by the Bonferroni post-hoc test (Table 8-1)

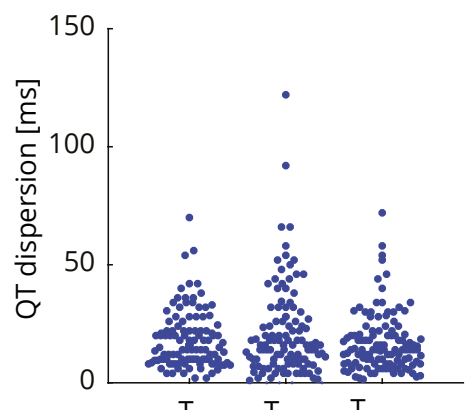

$T_{-1 d} \quad T_{+1 d} \quad T_{+3 m}$

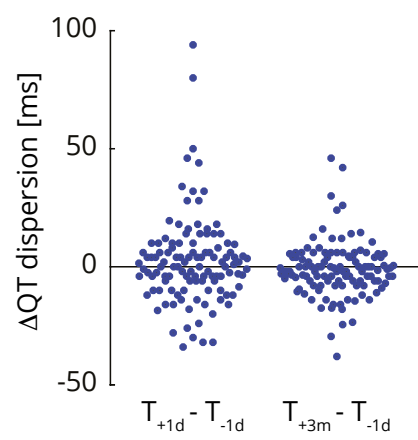

$T_{+1 d}-T_{-1 d} \quad T_{+3 m}-T_{-1 d}$
Figure 8-2 QT dispersion before and after pulmonary vein isolation. $T_{-10^{\prime}}$ one day prior to the procedure; $T_{+1 d^{\prime}}$ one day post procedure; $T_{+3 m^{\prime}} 3$ months post procedure.

\subsection{Discussion}

The objective of this study was to investigate the impact of PVI procedure on ventricular APD and heart rate since a change between these two might be an underlying cause of the increased burden of VPBS and NSVTs which are seen in a small number of AF patients following PVI-12.

The results of this study show that the RR-interval significantly decreases after a PVI. This is in line with an earlier study who have reported an increase in heart rate after a AF ablation due to a reduction of cholinergic control9. In normal physiological circumstances, the QT-interval should adapt to this shortening in RR-interval|'. In our study the QT-interval indeed significantly shortens in line with the RR-interval. Both QT and RR are shortest at one day post-procedure but remain shortened at three months post-procedure. No significant within-subject differences in QTc were seen for any of the recording times which suggests that PVI has no significant impact on the capability of the ventricular APD (measured by the QT-interval) to adapt to the higher heart rates. Whether this is because PVI does not modulate the GP (enough) or whether GP modulation does not induce a change in the relationship between ventricular APD and heart rate cannot be concluded from this study.

Jungen et al. have shown that a small subset of their patients ( $n=6$ out of 111) have an increased VPB burden after a PVI9. In these six patients with increased VPB burden, QT dispersion was significantly higher post-PVI on average'. However, whether QT dispersion can be used to estimate the risk of an increased VPB burden is not clear from this observation. It is also not clear whether an increased QT dispersion leads to an increased VPB burden or whether an increased VPB burden leads to an increased QT dispersion. Our results show that the post-PVI QT dispersion of these six patients reported by Jungen et al. ( $40 \pm 6 \mathrm{~ms})$ are within the range of QT dispersion measure before the PVI. Since Jungen compared only six patients with an increased VPB burden with six age- and gender-matched controls, chances are that among the remaining patients some also have an increased QT dispersion without an increase in VPB burden. Our results show no significant difference between the QT dispersion before and after a PVI treatment for the entire study population indicating that, on average, PVI does not affect the ventricular repolarization heterogeneity. It has furthermore to be noted that QT dispersion is a very laborious measure. It therefore is easily affected by biphasic T-waves, flat T-waves and prominent U-waves. Furthermore, a broad range of QT dispersions has been reported in healthy populations ${ }^{21}$. We therefore doubt the usefulness of this measure and would advise to study T-wave morphology differences between leads instead of the QT dispersion.

The mechanism(s) behind the increased susceptibility for VA after PVI remains unclear. Apart from the hypothesis that GP modulation causes the increased susceptibility, others have hypothesized that propafenone therapy for AF was associated with new-onset VA in AF patients ${ }^{22}$. The results of a study by Wu et al. does however not support this finding since propafenone was not prescribed in their study and patients still show new-onset VA post-PV|12. The only measure associated with new-onset VA in the study of Wu et al. was an increased serum leukocyte count which implies that an inflammatory response caused by the PVI may be an underlying mechanism of $\mathrm{VA}^{12}$. Patel et al. showed a significant difference in post-PVI ejection fraction and heart rate between patients that developed VPB and patients who did not ${ }^{10}$. However, since the ejection fraction remained in the normal range and increased post-PVI heart rates have been described in larger cohorts7,8, the clinical relevance of these finding are unclear. 
In this study we used Fridericia's formula to correct the QT-interval for heart rate. Interestingly, the QT correction method used, influences the results. Although Bazett's formula has been shown to be inferior to Fridericia's (because Bazett's formula is known to overestimate QTc with an RR-interval $\leq 1000 \mathrm{~ms}$ and underestimates QTc with an RR-interval $<1000 \mathrm{~ms}^{19}$ ) we also tried Bazett's formula in this study and found a significant increase in the QTc calculated with Bazett's formula after PVI. This can however be explained by the fact that half of our study population had an RR-interval $\geq 1000 \mathrm{~ms}$ prior to the PVI which shortened to $\leq 1000 \mathrm{~ms}$ as a result of the PVI. The increase in QTc as a result of the PVI therefore could be a result of the QTc overestimation of Bazett. Another remarkable coincidental finding of this study is the broad range of QTC within one patient over time. From long QT syndrome studies, it is known that the QT-interval may vary over time and therefore hampers the diagnosis of the long-QT syndrome ${ }^{23}$. Our data shows that this also applies in a study cohort not suspected of long-QT syndrome.

\subsubsection{Study limitations}

This study has several limitations due to the observational design of the registry. First of all, the cohor might be biased because we only included patients who were in sinus rhythm during all three ECG recordings. This might explain the relatively high number of re-do patients in this cohort. Secondly, because the cohort consists of many re-do patients, the QT-interval prolonging mechanism of PVI might already have taken place after the first procedure. However, the univariate analysis of variance showed that there was no difference in QTc at $\mathrm{T}_{+3 \mathrm{~m}}$ with respect to $\mathrm{T}_{-1 \mathrm{~d}}$ between re-do and first-time procedure patients. The high recurrence rate in this cohort, however, might be explained by the high number of re-do patients. Since a lot of patients needed a re-do procedure, these might be complex AF patients Thirdly, we did not look into QT prolonging drug use in this study. However, following the institution's standard of care, all antiarrhythmic drugs (if any) were continued through the entire period of this study. Therefore, drug use will most likely not alter the results. Finally, the self-reported AF recurrence of course has its limitations. Patients might suffer from silent AF or might interpret other arrhythmias as AF. Because many patients were referred back to their treating physicians in other hospitals, we do not have access to 24-hour Holter reports and can therefore not address this issue.

\subsection{Conclusion}

In this study, we showed that pulmonary vein isolation is not resulting in a change in the relationship between heart rate and ventricular action potential duration as objectivated by the absence of change in QTc after this procedure. Furthermore, the change in QTC at three months post-PVI did not differ between sex, ablation techniques, redo or index procedures, self-terminating or non-self-terminating AF and recurrence or no recurrence.

\subsection{References}

Ng, G. A., Brack, K. E., Patel, V. H. \& Coote, J. H. Autonomic modulation of electrical restitution alternans and ventricular fibrillation initiation in the isolated heart. Cardiovasc. Res. 73, 750-760 (2007).

2. Zipes, D. P. \& Rubart, M. Neural modulation of cardiac arrhythmias and sudden cardiac death. Heart Rhythm 3, 108-113 (2006).

3. Pauza, D. H., Skripka, V., Pauziene, N. \& Stropus, R. Morphology, distribution, and variability of the epicardiac neural ganglionated subplexuses in the human heart. Anat. Rec. 259, 353-382 (2000).

4. Armour, J. A. Potential clinical relevance of the 'little brain' on the mammalian heart. Exp. Physiol. 93, 165-176 (2008).

5. Armour, J. A., Murphy, D. A., Yuan, B. X., Macdonald, S. \& Hopkins, D. A. Gross and microscopic anatomy of the human intrinsic cardiac nervous system. Anat. Rec. 247, 289-298 (1997).

6. Pappone, C. et al. Pulmonary Vein Denervation Enhances Long-Term Benefit after Circumferential Ablation for Paroxysmal Atrial Fibrillation. Circulation 109, 327-334 (2004).

7. Wickramasinghe, S. R. \& Patel, V. V. Local Innervation and Atrial Fibrillation. Circulation 128, 15661575 (2013)

8. Hsieh, M., Chiou, C., Wen, Z. \& Wu, C. Alterations of Heart Rate Variability After Radiofrequency. Online 2237-2243 (1999). doi:10.1161/01.CIR.100.22.2237

9. Jungen, C. et al. Disruption of cardiac cholinergic neurons enhances susceptibility to ventricular arrhythmias. Nat. Commun. 8, (2017).

10. Patel, P. J. et al. Outflow tract premature ventricular depolarizations after atrial fibrillation ablation may reflect autonomic influences. J. Interv. Card. Electrophysiol. 41, 187-192 (2014).

11. Münkler, P. et al. Ventricular tachycardia (VT) storm after cryoballoon-based pulmonary vein isolation. Am. J. Case Rep. 19, 1078-1082 (2018).

12. $\mathrm{Wu}$, L. et al. New-onset ventricular arrhythmias post radiofrequency catheter ablation for atrial fibrillation. Med. (United States) 95, 1-5 (2016).

13. He, B. et al. Effects of ganglionated plexi ablation on ventricular electrophysiological properties in normal hearts and after acute myocardial ischemia. Int. J. Cardiol. 168, 86-93 (2013).

14. Tse, G., Chan, Y. W. F., Keung, W. \& Yan, B. P. Electrophysiological mechanisms of long and short QT syndromes. IJC Hear. Vasc. 14, 8-13 (2017)

15. Straus, S. M. J. M. et al. Prolonged QTC interval and risk of sudden cardiac death in a population of older adults. J. Am. Coll. Cardiol. 47, 362-367 (2006).

16. Franz, M. R., Swerdlow, C. D., Liem, L. B. \& Schaefer, J. Cycle length dependence of human action potential duration in vivo. Effects of single extrastimuli, sudden sustained rate acceleration and deceleration, and different steady-state frequencies. J. Clin. Invest. 82, 972-979 (1988).

17. Hermans, B. J. M. et al. The development and validation of an easy to use automatic QT-interval algorithm. PLoS One 12, e0184352 (2017).

18. Pan, J. \& Tompkins, W. J. A real-time QRS detection algorithm. IEEE Trans. Biomed. Eng. 32, 230-6 (1985).

19. Vandenberk, B. et al. QT correction across the heart rate spectrum, in atrial fibrillation and ventricular conduction defects. PACE - Pacing Clin. Electrophysiol. 41, 1101-1108 (2018).

20. Luo, S., Michler, K., Johnston, P. \& MacFarlane, P. W. A comparison of commonly used QT correction formulae: The effect of heart rate on the QTc of normal ECGs. I. Electrocardiol. 37, 81-90 (2004).

21. Malik, M. \& Batchvarov, V. N. Measurement, interpretation and clinical potential of QT dispersion. J. 
Am. Coll. Cardiol. 36, 1749-1766 (2000).

22. Lin, C. Y. et al. Factors predisposing to ventricular proarrhythmia during antiarrhythmic drug therapy for atrial fibrillation in patients with structurally normal heart. Heart Rhythm 12, 1490-1500 (2015).

23. Viskin, S. The QT interval: Too long, too short or just right. Heart Rhythm 6, 711-715 (2009). 


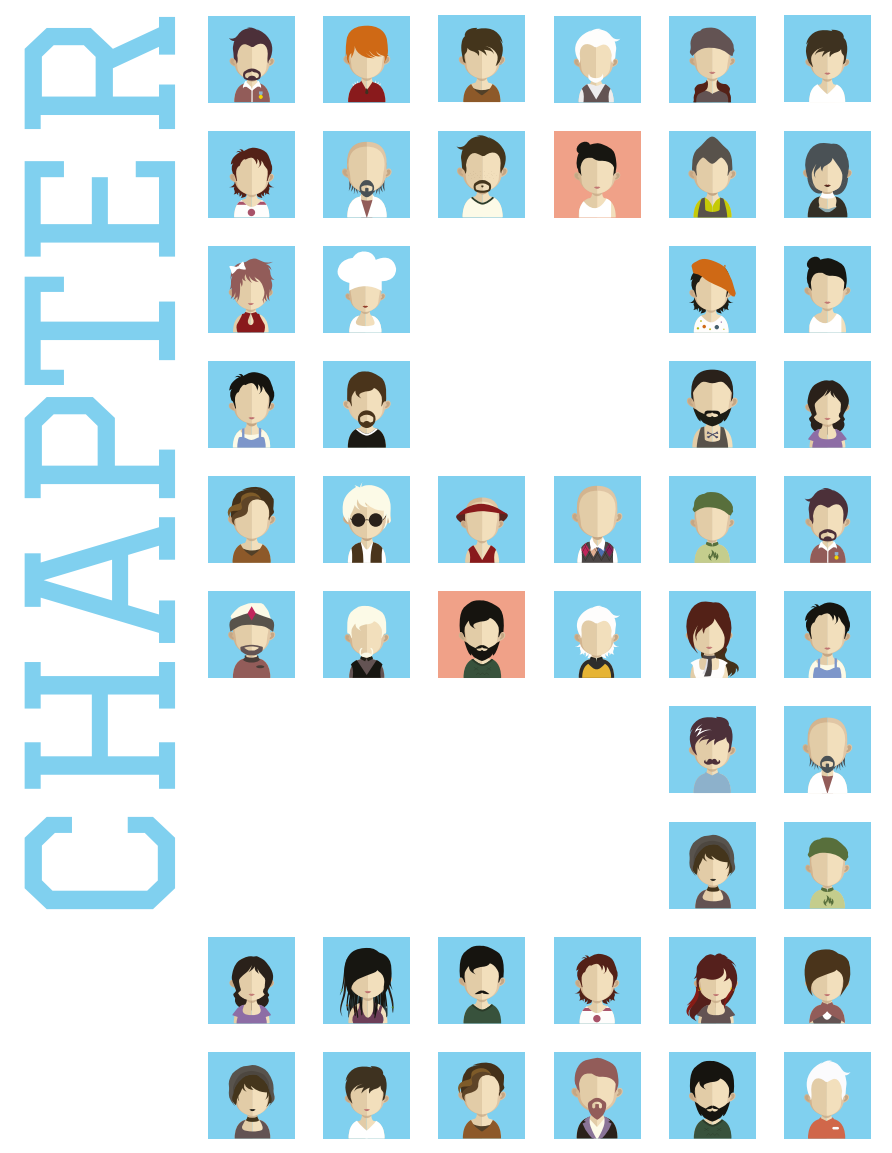

\section{CHAPTER 9}

EFFECTS OF DIFFERENT DIALYSATE CALCIUM CONCENTRATIONS AND CITRIC-ACID DIALYSATE ON QTC

Karlien J. ter Meulen*, Ben J.M. Hermans*, Frank M. van der Sande, Bernard Canaud, Jeroen P. Kooman, Tammo Delhaas

Under review at Blood Purification 
Abstract

\section{Background}

Lower dialysate calcium (dCa) concentration and dialysate citric-acidification may positively affect calcification propensity in serum of haemodialysis (HD) patients. However, the accompanying lower onized blood calcium concentration may lead to a prolonged cardiac action potential, which is possibly pro-arrhythmic.

\section{Objectives}

The aim of this study is to investigate the influence of dCa and citric-acidification on the continuously measured QTc.

Methods

We conducted a four-week multicentre, randomized cross-over trial in which chronic and haemodynamic stable HD patients had continuous ECG monitoring during their thrice weekly HD sessions. In the first and third week (wash-out) they received acetic-acid dialysate with a dCa of $1.50 \mathrm{mmol} / \mathrm{l}$ (A-Ca1.50), in the second week acetic-acid dialysate with a dCa of $1.25 \mathrm{mmol} / \mathrm{l}$ (A-Ca1.25) or citric-acid dialysate with a dCa of $1.50 \mathrm{mmol} / \mathrm{l}$ (C-Ca1.50) depending on randomization. In the fourth week, patients received the opposite treatment of week two. HD sessions were bicarbonatebased.

Results

Out of 20 participating patients, the continuous ECG-data of $13 \mathrm{HD}$ patients were available for analysis. It showed a significant though limited increase of QTc-interval with A-Ca1.25 (from 431 to $449 \mathrm{~ms}$ (start to end); $p<0.001$ ) and with C-Ca1.50 (from 427 to $444 \mathrm{~ms} ; \mathrm{p}=0.007$ ), but not during A-Ca1.50 (from 439 to $443 \mathrm{~ms} ; \mathrm{p}=0.13$ ). There were no differences in electrolytes composition otherwise between the dialysis fluids.

\section{Conclusion}

In conclusion, we found that the use of C-Ca1.50 or A-Ca1.25 is associated with a significantly prolongation of QTC that was however relatively limited.

\subsection{Introduction}

Although lower dialysate calcium concentrations (dCa) may positively affect the calcification tendency in serum of haemodialysis (HD) patients, they potentially have a prolonging effect on cardiac action potential. International guidelines are conflicting upon proposed dCa. Whereas a dCa of $1.25 \mathrm{mmol} / \mathrm{I}$ is more common in the United States, in Europe and Japan also a dCa of $1.50 \mathrm{mmol} / \mathrm{l}$ is frequently used. Higher dCa is associated with an increased risk for all-cause mortality, while lower dCa $(<1.25 \mathrm{mmol} / 1)$ is associated with a higher risk for sudden cardiac death and arrhythmia ${ }^{1,2}$. The most common dialysis fluid in bicarbonate dialysis is based on a combination with acetate which even in small concentrations is hypothesized to have an effect on haemodynamic stability during dialysis ${ }^{3}$. The recently developed citric-acid dialysate (dCit) showed a positive influence on the dialysis efficiency while haemodynamic stability was possibly improved ${ }^{4,5}$

HD is associated with a risk of prolongation of the heart rate-corrected QT-intervals (QTC) ${ }^{6.7}$. It is known that citrate chelates ionized calcium (iCa) and magnesium. The lower iCa effects cardiac repolarisation and eventually can lead to increased QTC and higher risk on arrhythmiar. However, general studies comparing different dCa concentrations only addressed differences in QTc between the start and end of dialysis, whereas to the best of our knowledge the effect of dCa on QTC during HD nor the effect of dCit on QTc has not been studied yet. We studied the influence of conventional bicarbonate dialysis combined with acetate-acid with dCa 1.50mmol/l (A-Ca1.50), dCa 1.25mmol/I (A-Ca1 25) and dCit with dCa $1.50 \mathrm{mmol} / /$ (C-Ca1.50) on QTC and RR-interval during the complete dialysis sessions. The hypothesis was that QTC would increase during HD with C-Ca1.50 and A-Ca1.25 as compared to A-Ca1.50.

\subsection{Methods}

\subsubsection{Study design}

This multicentre, randomized cross-over trial was conducted in two Dutch hospitals (Catharina hospital Eindhoven and Maastricht Medical University Centre). Chronic and stable HD patients were recruited between April and September 2017. Patients were on thrice weekly HD for at least three months, had a stable blood access and a QTc below 470ms recorded by a 12-lead ECG at inclusion. Patients with acute ongoing illness, malignancy or uncontrolled diabetes were excluded.

Patients received A-Ca1.50 in the first week, in the second week either A-Ca1.25 or C-Ca1.50 depending on randomization, in the third week A-Ca1.50 as a washout and in week four the opposite treatment of the second week. All patients used a high-flux membrane and maintained the same blood- and dialysate flow during the study. They all received thrice weekly HD sessions between three to four hours with a Fresenius 5008 Therapy System dialysis machine (Fresenius Medical Care, Bad Homburg, Germany). Baseline characteristics were attained from medical files.

\subsubsection{Dialysate composition}

HD sessions were bicarbonate-based. All three dialysis fluids consisted of $138.0 \mathrm{mmol} / \mathrm{l}$ sodium, 0.5 $\mathrm{mmol} / \mathrm{l}$ magnesium and $1.0 \mathrm{~g} / \mathrm{lg}$ glucose. The calcium (Ca) concentration was either $1.25 \mathrm{mmol} / \mathrm{I}$ (A-Ca1.25) 
or $1.50 \mathrm{mmo} / /$ (A-Ca1.50 and C-Ca1.50). Whereas A-Ca1.25 and A-Ca1.50 contained $3.0 \mathrm{mmol} / /$ acetate, the acetate was replaced with $1.0 \mathrm{mmol} / \mathrm{I}$ citrate in C-Ca1.50. Potassium $(2-3 \mathrm{mmol} / \mathrm{l})$ and bicarbonate (provided with Bibag $\circledast$, range $30-36 \mathrm{mmol} / /$ ) were individualized, but the concentrations did not change during the study. All used dialysates are registered products that are common in daily practice. All patients had A-Ca1.50 as their regular dialysate.

\subsubsection{Electrocardiography analysis}

The Task Force Monitor (TFM, CN Systems, Austria) was used to record ECG during dialysis sessions. To improve ECG signal-to-noise ratio, a median complex was constructed for every two minutes by aligning all complexes within these two minutes on the R-peak. OT-intervals were determined offline using an automated tangent approach by a custom-made algorithm in MATLAB (2017a, Mathworks, Natick, MA,

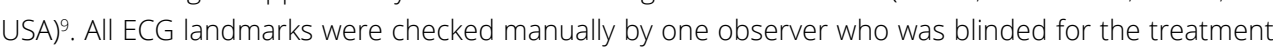
QTc was calculated using Bazett's formula ${ }^{10}$. The first median complex (i.e. first two minutes after start dialysis) was used as the baseline (QTc $c_{\text {Basenen }}$ ). For each hour of dialysis, median QTc (QTc $c_{1 \prime} \mathrm{QTC}_{2 h}$ etc) and $R R$-intervals ( $R R_{1 n}, R_{2 h}$ etc) were calculated. All recordings were made during the second or third session of week one, two and four in order to omit the effects of a long interdialytic period on the haemodynamic response during $\mathrm{HD}$

\subsubsection{Statistical analysis}

The derived data were analysed using IBM SPSS Statistics for Windows version 23.0 (IBM Corp. Armonk NY, USA). Data was expressed as median with interquartile range [25th; 75th percentile]. Due to the small sample size, non-parametric testing was applied (Friedman and Wilcoxon Signed Rank test). All tests were two-tailed and a p-value $<0.05$ was considered statistically significant. No additional correction for multiplicity was used.

\subsection{Results}

Out of the 20 patients, 7 patients were excluded because of missing recordings ( $n=3$ ) or cardiologic disorders that may affect QTC (atrial fibrillation $(n=1)$, repolarisation disorders $(n=2)$ and frequent ventricular extrasystoles $(n=1))$. Patients had a median age of $69[51 ; 75]$ years, 7 were male $(53.8 \%)$ and the median dialysis vintage was $25[7.5 ; 66]$ months. Out of the 13 patients, causes of renal failure were renal vascular disease $(n=5 ; 38.5 \%)$ of which $2(15.4 \%)$ were due to hypertension, diabetes mellitus type $2(n=2 ; 15.4 \%)$, congenital renal dysplasia with urinary tract malformation $(n=1 ; 7.7 \%)$, membranoproliferative glomerulonephritis $(n=1 ; 7.7 \%)$, chronic renal failure aetiology unknown $(n=1 ; 7.7 \%)$ and other $(n=3 ; 23.1 \%)$. A total of $12(92.3 \%)$ patients were diagnosed for hypertension and $3(23.1 \%)$ are current smokers.

\subsubsection{QTC}

Data are expressed in Table 9-1. Baseline QTc did not differ significantly between the three dialysis fluids. For A-Ca1.25, there was a significant increase at QTc $c_{1 h}, \mathrm{QTC}_{2 h}, \mathrm{QT} \mathrm{c}_{3 \mathrm{~h}}$ and $\mathrm{QTC} \mathrm{c}_{4 h}$ as compared to baseline ( $p=0.002 ; p=0.005 ; p=0.009 ; p=0.006$ respectively; not shown). Furthermore, $Q T c_{2 n}$ and $\mathrm{QTC} c_{3 \mathrm{~h}}$ significantly increased from $\mathrm{QTC} c_{1 h}(\mathrm{p}=0.02$ and $\mathrm{p}=0.04$; not shown). For $\mathrm{C}-\mathrm{Ca1} .50$, there was also a significant increase of QTc between baseline and $\mathrm{QTC}_{1 \mathrm{~h}^{\prime}} \mathrm{QTC}_{2 \mathrm{~h}^{\prime}} \mathrm{QTC}_{3 \mathrm{~h}}$ and $\mathrm{QTC}_{4 \mathrm{~h}}(\mathrm{p}=0.007 ; \mathrm{p}=0.01$; $\mathrm{p}=0.003 ; \mathrm{p}=0.006$; not shown

There were no significant differences in QTc between the various hours of HD for A-Ca1.50. For A-Ca1.25 the largest QTc change was between QT $c_{1 n}$ and QT $c_{2 h}$, and the second biggest QTc change was found between QTCBaseline and QTC $c_{1 \text { h }}$. For C-Ca1.50 the largest QTc change was between QTC Baseline and QTc. In general, the largest rise in QTc was observed during the first hours of dialysis and was more pronounced with A-Ca1.25 and C-Ca1.50.

\begin{tabular}{|c|c|c|c|c|c|}
\hline & & A-Ca1.50 & A-Ca1.25 & C-Ca1.50 & p-value* \\
\hline \multirow{6}{*}{ 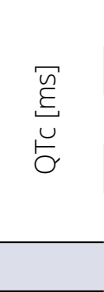 } & QTC $C_{\text {Baseline }}$ & $439[415 ; 447]$ & $431[397 ; 451]$ & $427[408 ; 438]$ & 0.37 \\
\hline & $\mathrm{QTC}_{1 \mathrm{~h}}$ & $439[418 ; 448]$ & $440[401 ; 456]$ & $434[416 ; 456]$ & 0.23 \\
\hline & $\mathrm{QTC}_{2 \mathrm{~h}}$ & $442[421 ; 452]$ & $448[409 ; 460]$ & $441[422 ; 457]$ & 0.50 \\
\hline & $\mathrm{QTC}_{3 \mathrm{~h}}$ & $447[422 ; 455]$ & $449[406 ; 463]$ & $443[430 ; 466]$ & 0.29 \\
\hline & $\mathrm{QTC}_{4 \mathrm{~h}}$ & $443[415 ; 460]$ & $449[408 ; 467]$ & $444[427 ; 467]$ & 0.23 \\
\hline & p-value & 0.13 & $<0.001$ & 0.007 & \\
\hline \multirow{4}{*}{ 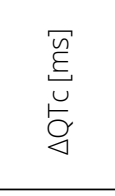 } & $\Delta \mathrm{QTc1}$ & $0[2 ; 9]$ & $5[3 ; 9]$ & $10[2 ; 17]$ & 0.15 \\
\hline & $\triangle \mathrm{QTC2}$ & $4[-9 ; 9]$ & $7[1 ; 13]$ & $4[-2 ; 13]$ & 0.58 \\
\hline & $\triangle \mathrm{QTC3}$ & $1[0 ; 9]$ & $1[-4 ; 7]$ & $4[-2 ; 9]$ & 0.50 \\
\hline & $\triangle \mathrm{QTC4}$ & $1[-5 ; 5]$ & $2[-1 ; 5]$ & $0[-3 ; 6]$ & 0.93 \\
\hline \multirow{3}{*}{ 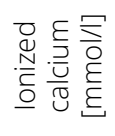 } & Predialysis & $1.07[1.14 ; 1.20]$ & $1.13[1.09 ; 1.21]$ & $1.14[1.06 ; 1.21]$ & 0.91 \\
\hline & Post dialysis & $1.23[1.20 ; 1.30]$ & $1.12[1.08 ; 1.17]$ & $1.10[1.01 ; 1.13]$ & $<0.001$ \\
\hline & Delta & $0.06[0.09 ; 0.13]$ & $0.0[-0.07 ; 0.02]$ & $-0.04[-0.08 ; 0.03]$ & $<0.001$ \\
\hline $\begin{array}{l}\text { Delta ic } \\
\text { p)*t }\end{array}$ & vs. delta C & $0.13 ; 0.70$ & $-0.49 ; 0.09$ & $-0.46 ; 0.12$ & \\
\hline
\end{tabular}

Table 9-1 Overview of QTC and ionized calcium per dialysate. Data are expressed as median with [25th; 75th percentile]. p-value is calculated with Friedman test. *p-value is calculated with Friedmand test between dialysates. ${ }^{*}{ }_{r}$ and $p$ are measured with Spearmans's correlation. A-Ca1.50 = aceticacid dialysate with calcium concentration $1.50 \mathrm{mmol} / \mathrm{l}$. A-Ca1.25= acetic-acid dialysate with calcium concentration $1.25 \mathrm{mmol} / /$. C-Ca1.50 = citric-acid dialysate with calcium concentration $1.50 \mathrm{mmol} /$. $\mathrm{QT} c_{\text {Baseline }}=\mathrm{QTc}$ of the first median complex (first 2 minutes of haemodialysis). QT $c_{1 h^{\prime}} \mathrm{QT} c_{2 h^{\prime}} \mathrm{QT} c_{3 h^{\prime}} \mathrm{QTC}_{4 \mathrm{~h}}$ = median QTc of respectively the first, second, third and fourth hour of haemodialysis. $\triangle \mathrm{QTC1}, \triangle \mathrm{QTC2}$, $\triangle \mathrm{QTC3}, \triangle \mathrm{QTC4}=\triangle \mathrm{QTC}$ of respectively between the first hour and baseline, second and first, third and second, and fourth and third hour of haemodialysis.

\subsubsection{RR-interval}

Only A-Ca1.25 showed a significant decrease of RR-interval from 869 [743; 975] ms at baseline till 794 712; 926] ms at fourth hour ( $p=0.02$ ), with significant differences between $R R_{\text {Baseine }}$ and $R R_{1,}, R R_{2,}, R R_{3}$ and $R R_{4 h}(p<0.05 ; p=0.03 ; p=0.01)$. There were no significant differences in $\triangle R R$ within and between the three dialysates (not shown). 


\section{QTc displayed by hour}

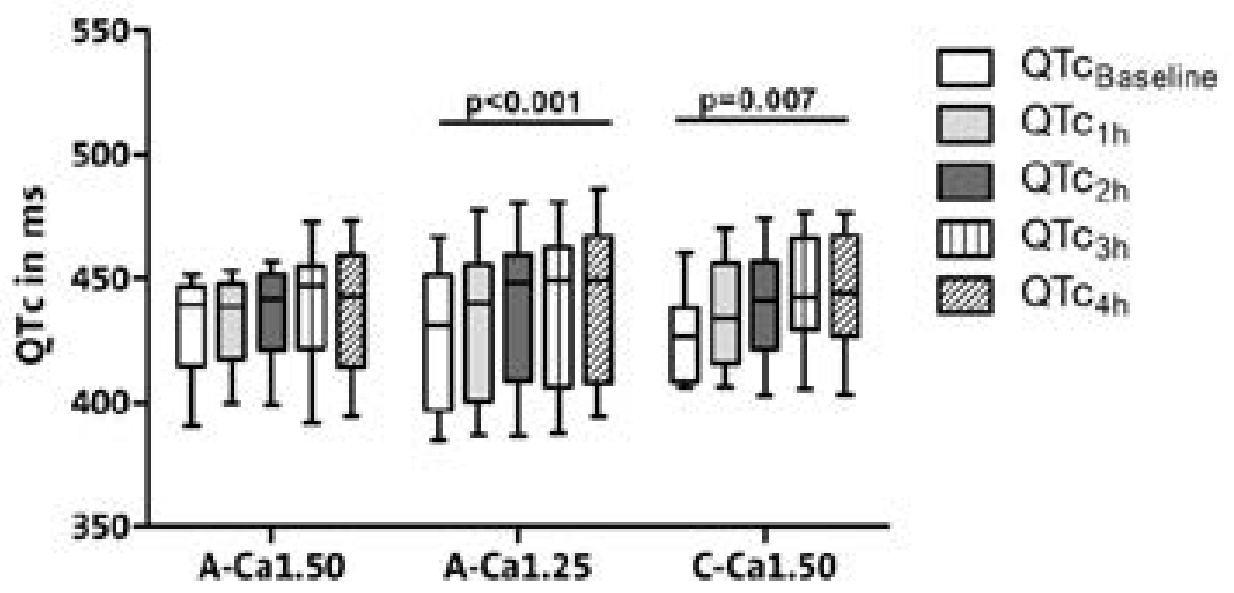

RR displayed by hour

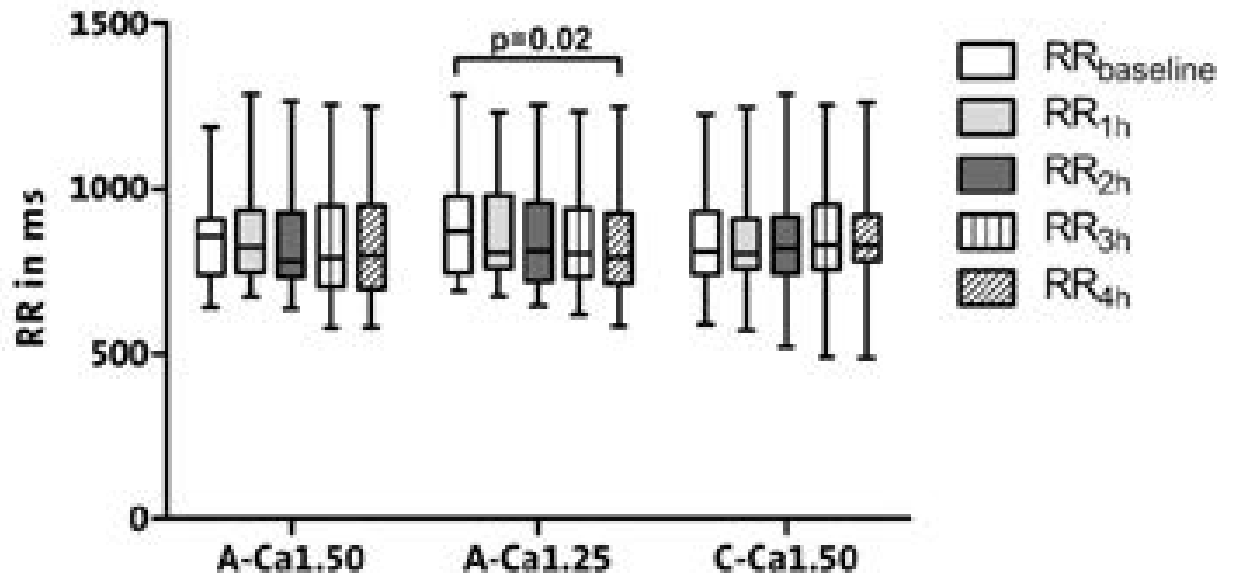

Figure 9-1 QTC and RR per hour. Data are expressed as median, interquartiles with minimum and maximum. A-Ca1.50 = acetic-acid dialysate with calcium concentration 1.50mmol/l. A-Ca1.25 = aceticacid dialysate with calcium concentration $1.50 \mathrm{mmol} / \mathrm{l}$. C-Ca1.50= citric-acid dialysate with calcium concentration 1.50mmo//I. QTC $c_{\text {Baseline }}=$ QTc of the first median complex (first 2 minutes of haemodialysis). $\mathrm{QTC}_{1,}, \mathrm{QTC}_{2 h}, \mathrm{QTC}_{3 n}, \mathrm{QTC} c_{4 h}=$ median QTc of respectively the first, second, third and fourth hour of haemodialysis. $\mathrm{RR}_{1 h^{\prime}} \mathrm{RR}_{2 h^{\prime}} \mathrm{RR}_{3 h^{\prime}}, \mathrm{RR}_{4 \mathrm{~h}}=$ median $\mathrm{RR}$-interval of respectively the first, second, third and fourth hour of haemodialysis. p-value was measured with Friedman's test

\subsubsection{Ionized calcium}

There is a significant difference in $\triangle$ ionized calcium (iCa) between the three dialysis fluids $(\mathrm{p}<0.001)$. It was significant different between A-Ca1.50 $(0.06[0.09 ; 0.13] \mathrm{mmol} / \mathrm{l})$ and A-Ca1.25 $(0.0[-0.07 ; 0.02]$ $\mathrm{mmol} / \mathrm{l} ; \mathrm{p}=0.002)$ but as well as between $\mathrm{A}-\mathrm{Ca} 1.50$ and $\mathrm{C}-\mathrm{Ca} 1.50(-0.04[-0.08 ; 0.03] \mathrm{mmol} / \mathrm{l} ; \mathrm{p}=0.002)$. There is no correlation between $\triangle \mathrm{i} C \mathrm{C}$ and $\triangle \mathrm{QT}$

\subsection{Discussion}

Our results showed that the QTc significantly increased during A-Ca1.25 and C-Ca1.50, but not during A-Ca1.50, albeit without a significant difference in the QTc between the dialysis fluids. The largest changes in QTc were seen during the first hours of treatment for C-Ca1.50 and for A-Ca1.25.

Whereas our study showed the largest increase of QTc in the first hours of the dialysis, Eldehni et al. stated that the haemodynamic stress reached its peak around the third to fourth hour of dialysis ${ }^{11}$. We suggest that the change of QTc is most likely caused by intra- and extracellular shifts of electrolytes, which are likely most pronounced at the start of dialysis because at this point in time the concentration gradient is the largest. Genovesi et al. showed that concentrations of calcium and potassium are associated with changes in ventricular repolarization duration ${ }^{12}$. They found a significant increase of QTC in the first hour of low dCa (1.25 mmol/l/) and low potassium (2mmol//) $)^{12}$. Another study in A-Ca1 25 observed a larger mean Ca loss in dialysate and ultrafiltrate at the beginning of the treatment as compared to its end ${ }^{13}$. In our study, there was no significant inverse correlation between $\triangle \mathrm{QTC}$ and $\mathrm{iCa}$ which is in contrast with other studies ${ }^{8}$. Our finding that the QTc remained mainly stable in A-Ca1.50 is in conformation with other studies ${ }^{8}$.

In C-Ca1.50 there was an increase in QTC during dialysis without a correlation with iCa. The difference might additionally be caused by a decline in ionized magnesium, which can also be caused due to the chelating effect of citric acid. Regrettably, we did not have the opportunity to assess ionized magnesium levels in our study.

A limitation of our study is its small study size. Furthermore, QTc-dispersion between leads could not be calculated since precordial ECG leads are not recorded. For future research, it might be relevant to follow-up QTC in the hours after HD because it is known that Ca may rebound up to 180 minutes after dialysis ${ }^{14}$. It might also be of added value to monitor at-risk patients with implanting devices such as Reveal LINQ for detecting arrhythmias 15,16

Although the effects on QTC appeared in general to be relatively minor, we suggest that this study can contribute to the clinical decision to personalize the optimal dialysate for each patient, which can be based on the calcification propensity, haemodynamic stability and the plasma- to- dialysate calcium ratio $^{14}$

In conclusion, we show that QTc significantly increases during haemodialysis with A-Ca1.25 and C-Ca1.50, but not with A-Ca1.50. Although not statistically different, the largest increase in QTC happens in the first hours of the dialysis. 


\subsection{References}

1. Pun, P. H., Lehrich, R. W., Honeycutt, E. F., Herzog, C. A. \& Middleton, J. P. Modifiable risk factors associated with sudden cardiac arrest within hemodialysis clinics. Kidney Int. 79, 218-227 (2011).

2. Brunelli, S. M., Sibbel, S., Do, T. P., Cooper, K. \& Bradbury, B. D. Facility dialysate calcium practices and clinical outcomes among patients receiving hemodialysis: A retrospective observational study. Am. J. Kidney Dis. 66, 655-665 (2015).

3. Movilli, E. et al. A prospective comparison of bicarbonate dialysis, hemodiafiltration, and acetatefree biofiltration in the elderly. Am. J. Kidney Dis. 27, 541-547 (1996).

4. Schmitz, M. et al. Effects of citrate dialysate in chronic dialysis: A multicentre randomized crossover study. Nephrol. Dial. Transplant. 31, 1327-1334 (2016).

5. Gabutti, L., Lucchini, B., Marone, C., Alberio, L. \& Burnier, M. Citrate- vs. acetate-based dialysate in bicarbonate haemodialysis: Consequences on haemodynamics, coagulation, acid-base status, and electrolytes. BMC Nephrol. 10, (2009).

6. Nie, Y. et al. Electrocardiographic abnormalities and QTC interval in patients undergoing hemodialysis. PLoS One 11,1-11 (2016).

7. Alabd, M. A., El-Hammady, W., Shawky, A., Nammas, W. \& El-Tayeb, M. QT Interval and QT Dispersion in Patients Undergoing Hemodialysis: Revisiting the Old Theory. Nephron Extra 1, 1-8 (2011).

8. Näppi, S. E., Virtanen, V. K., Saha, H. H. T., Mustonen, J. T. \& Pasternack, A. I. QT(c) dispersion increases during hemodialysis with low-calcium dialysate. Kidney Int. 57, 2117-2122 (2000).

9. Hermans, B. J. M. et al. The development and validation of an easy to use automatic QT-interval algorithm. PLoS One 12, e0184352 (2017)

10. Bazett, H. C. An analysis of the time-relation of electrocardiograms. Heart 7, 353-370 (1920).

11. Eldehni, M. T., Odudu, A. \& McIntyre, C. W. Characterising haemodynamic stress during haemodialysis using the extrema points analysis model. Nephron - Clin. Pract. 128, 39-44 (2014).

12. Genovesi, S. et al. Electrolyte concentration during haemodialysis and QT interval prolongation in uraemic patients. Europace 10, 771-777 (2008)

13. Fernandez, E., Borras, M., Pais, B. \& Montolio, J. Low-calcium dialysate stimulates parathormone secretion and its long-term use worsens secondary hyperparathyroidism. J. Am. Soc. Nephrol. 6 , 132-135 (1995).

14. Maduell, F. et al. Individualización del calcio en el baño de diálisis: Una asignatura pendiente. Nefrologia 32, 579-586 (2012).

15. Pürerfellner, $H$. et al. Miniaturized Reveal LINQ insertable cardiac monitoring system: First-inhuman experience. Heart Rhythm 12, 1113-1119 (2015).

16. Charytan, D. M. et al. Arrhythmia and Sudden Death in Hemodialysis Patients: Protocol and Baseline Characteristics of the Monitoring in Dialysis Study. Clin. J. Am. Soc. Nephrol. 11, 721-734 (2016). 


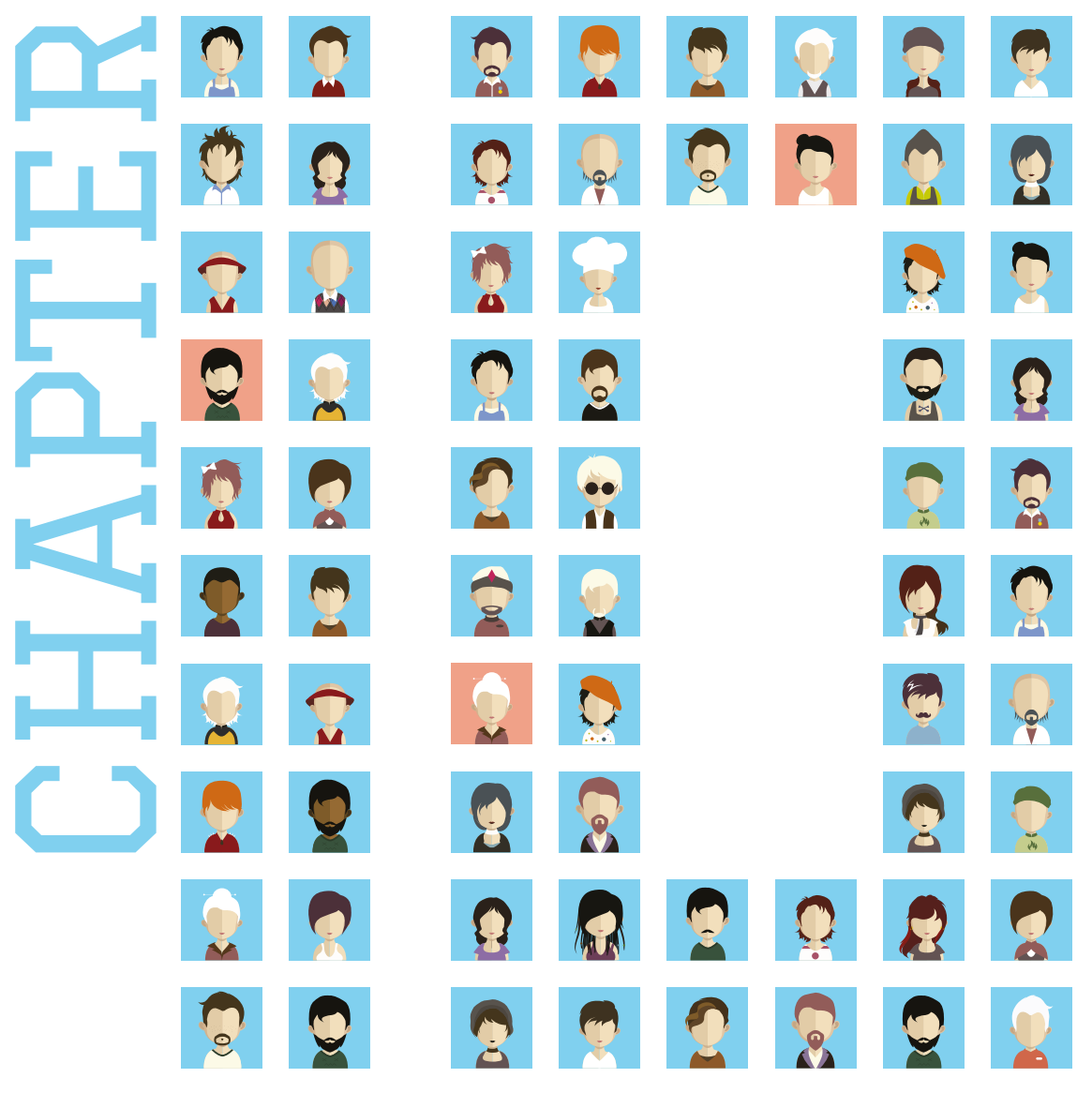

CHAPTER 10

GENERAL DISCUSSION 


\subsection{Introduction}

In this final chapter, the results, limitations and clinical relevance of the studies presented in this thesis are linked and put in broader perspective. To that end, this chapter is divided into paragraphs that discuss topics that either hamper or could aid in the diagnosis of LQTS. The following two paragraphs (Paragraph 10.2 and 10.3) of this chapter discuss what to keep in mind when measuring and interpreting the QT-interval and also briefly touches upon more sophisticated parameters derived from QT-interval measurements. The added value of the T-wave morphology in the diagnosis of LQTS will be discussed in Paragraph 10.4. Paragraph 10.5 reviews current issues with gold standard for the diagnosis of LQTS Finally, future perspectives for the diagnosis of LQTS are discussed in the last paragraph of this chapter (Paragraph 10.6.

\subsection{Measuring the QT-interval}

\subsubsection{Manual assessment of the QT-interval}

The assessment of the QT-interval on standard 10-second electrocardiograms (ECG) has been used worldwide for decades to evaluate the risk of sudden cardiac death due to LQTS ${ }^{1,2}$. Traditionally, lead II is often referred to as the best lead to measure the QT-interval from ${ }^{3.4}$. Although all modern ECG recording systems provide users with a QT-interval and a QT-interval corrected for heart rate (QTC), cardiologists are often trained not to rely on automatically assessed QT-intervals and therefore often measure the interval by hand. Unfortunately, many physicians (including cardiologists) cannot accurately estimate QT and/or calculate a QTc and can therefore not correctly identify a prolonged QT-interval or QTC. Both underestimation of the QTc of patients with LQTS and overestimation of the QTC of healthy patients were common ${ }^{5}$. Driven by this distressing finding, Postema et al. reintroduced the tangent approach to correctly and reliably determine the end of the T-wave ${ }^{6,7}$, since determining the end of the T-wave is the hardest part of measuring a QT-interval. In another approach used to determine the end of the T-wave, the end is defined as the intersection of the terminal limb of the T-wave with the isoelectric baseline Several studies compared the tangent and threshold approach head-to-head and concluded that both methods are more or less equally reliable and reproducible in determining the end of the T-wave ${ }^{8-11}$. Still, we would advise to use the tangent approach since even inexperienced ECG readers were able to measure QT-intervals accurately when using the tangent approach?

A general downside of a manual QT-interval assessment is that it is time consuming wherefore, many physicians will measure one complex and use only one ECG lead to do so. Since QT-intervals can change over time as well as between ECG leads, the chosen complex as well as the used ECG lead influence the measured QT-interval. We therefore advise that if manual QT-interval assessment is needed, measure QT-intervals in multiple leads and complexes, especially when dealing with a borderline prolonged QT-interval.

\subsubsection{Automatic QT-interval measurement algorithms}

QT experts still question the accuracy of automatic measurements and recommend to supplement these measurements with manual reading ${ }^{4}$. This distrust might be fed by a limited number of extreme cases. Moreover, the current use of automatic algorithms can be improved. First of all, the algorithms embedded in the ECG recording systems often are a black box. Understanding why and in which cases 
the determination of the QT-interval might be erroneous might improve users' confidence. Secondly, not only the algorithm is a black box, also the detected landmarks (QRS onset and T-wave end) are often not provided with the ECG by the systems. Users can therefore not appreciate the performance in a blink of the eye by checking whether landmarks are correctly placed but have to manually estimate the QT-interval instead. The third unknown in many algorithms is the used complex. The QT-interval is often determined on an averaged complex over time (cf. Appendix Kligfield et al. ${ }^{12}$ ) which is not provided by standard ECG exports. All manual reassessments therefore assess other complexes which might lead to other QT-intervals and once more feeds the idea that algorithms are questionable. Manufacturers should provide details of their algorithms and show the landmarks used to calculate the intervals from in standard ECG exports.

Custom-made algorithms are widely available, but also have some general limitations. First of all, custommade algorithms are often published in technical journals not read by physicians and therefore these algorithms do not make it to the clinics. Secondly, details are often not provided wherefore implementing the algorithm by others Is not straightforward. Cardiology departments furthermore often lack technical knowledge to implement custom-made algorithm. Finally, the most important limitation might be the missing or poor validation of many custom-made algorithms. The community should invest in developing and validating a reliable open-source algorithm that can easily been used by clinicians all over the world.

\subsection{Diagnosing LQTS using the QT-interval}

\subsubsection{QT cut-offs}

In genetically confirmed LQTS, genetic mutations might lead to malfunction of ion channels thereby delaying ventricular repolarization². This delayed ventricular repolarisation is reflected by a prolonged QT-interval on the body surface ECG. Unfortunately, a prolonged QT-interval is not always caused by LQTS and a normal QT-interval does not always imply that the patient does not have LQTS ${ }^{13}$. The considerable overlap in QTc between LQTS patients and healthy controls have been shown in multiple studies $8,13,14$ including the studies shown in this thesis (Chapters 3 - 8). This considerable overlap is one of the main reasons for the troublesome diagnosis of LQTS.

Current international guidelines mainly focus on QTc cut-offs in the diagnosis of LQTS15,16. The latest EHRA guidelines recommend a QTC cut-off of $\geq 480 \mathrm{~ms}$ in repeated ECGS or an LQTS risk score ${ }^{17} \geq 3$ for clinical diagnosis of LQTS in asymptomatic patients ${ }^{16}$. LQTS should further be considered in the presence of a QTc $\geq 460 \mathrm{~ms}$ in patients with an unexplained syncopal episode ${ }^{16}$. Data from the cohort used in Chapters 3 - $\mathbf{7}$ of this thesis illustrates that a large number of LQTS patients with confirmed pathogenic variants in KCNQ1, KCNH2, or SCN5A have QTc < 460ms. Apart from genetic testing, these patients can only be diagnosed using the LQTS risk score according to the guidelines. However, since QTc is incorporated in the LQTS risk score, reaching an LQTS risk score $\geq 3$ with a QTc $<460 \mathrm{~ms}$ is difficult ${ }^{18}$. Many LQTS patients will therefore not be diagnosed if one applies the diagnostic criteria recommended in the latest EHRA guidelines. The diagnostic criteria in the guidelines should therefore be updated. Furthermore, the guidelines should elaborate on the issues with diagnosing LQTS solely based on QTc to warn physicians unfamiliar with these limitations and the possible consequences.
The guidelines furthermore do not elaborate on what method should be used to assess the QT-interva nor do they elaborate on the heart rate correction method. A comprehensive analysis of a large cohort of LQTS patients and controls showed that diagnostic cut-offs should be specified both for the method used to determine the QT-interval as well as for the method used to calculate the QTc with ${ }^{8}$. The best known and most frequently used QTC correction in both clinical practice and research is Bazett's formula: $\mathrm{QTc}=\mathrm{QT} / \mathrm{RR}^{1 / 2}$ (with QT in milliseconds and RR in seconds) ${ }^{19}$. However, Bazett's formula overestimates QT at high heart rates and underestimates QTC at low heart rate ${ }^{20}$. The QTc correction formula proposed by Fridericia $\left(\mathrm{QTC}=\mathrm{QT} / \mathrm{RR}^{1 / 3}\right)$ has been shown to be superior to Bazett by several studies ${ }^{20-22}$. The result from Chapter $\mathbf{8}$ is a clear example of possible clinical implications of using Bazett's correction method: whereas the QTc calculated using Bazett's formula showed a significant increase after a pulmonary vein isolation, the QTc using Fridericia's formula did not show any significant difference in QTc. The significant increase in QTC Bazett can be explained by the increase in heart rate in halve of the study cohort and the fact that Bazett is known to overestimate QTc at high heart rates. If we wouldn't have used Fridericia's formula as well, the conclusion of this study would be completely different. Although we do encourag the use of Fridericia's formula to correct QT-intervals for heart rate, one should keep in mind that all QT cut-offs derived from studies using Bazett's formula cannot be applied on QTC calculated with the use of Fridericia's formula. In summary, consensus on what heart rate correction method should be used should be reached. Since Fridericia has been shown to be superior to Bazett and is as easy to calculate as Bazett, we suggest shifting to Fridericia as the standard heart rate correction method. As a consequence of that, QTc cutoffs should be adjusted for Fridericia's method.

The QTc furthermore is affected by other influences such as age, sex, and hormones ${ }^{23}$. Therefore, in Chapter $\mathbf{7}$ we described a support vector machine model which is trained to identify LQTS patients based on the inputs age, sex and QTc. We showed that this model improved the diagnosis of LQTS tremendously when compared with two often mentioned QTc cut-offs from $>480 \mathrm{~ms}$ and $\geq 450 / 460 \mathrm{~ms}$ for males/females. Large clinical studies on the possible added value of sex and age-based QT-interval cut-offs should be performed and depending on the results, QT-interval cut-offs might have to be age- and sex-specific

\subsubsection{QT dynamics}

Cardiac events are often triggered by physical or emotional stress in LQTS genetic subtypes LQT-1 and LQT-21,24. Therefore, studying the QT-interval dynamic behaviour during circumstances that mimic arrhythmogenic triggers instead of using a standard 10-second resting ECG might improve LQTS diagnosis. Three provocation tests that improve LQTS diagnosis have been described: exercise test5 ${ }^{25-28}$, epinephrine infusion ${ }^{29-31}$ and the brisk standing test ${ }^{32,33}$. A general downside of these studies is that they all (except on $\mathrm{e}^{30}$ ) rely on manually assessed QT-interval measurements and therefore only measure limited number of complexes with the inevitable consequence that information is lost. In Chapter 4, we applied our QT-interval algorithm from Chapter 2 on ECGs obtained during brisk standing tests and extracted the same parameters as the initial studies $\mathrm{did}^{32,33}$. Although we were unable to reproduce the diagnostic accuracy of this initial studies, a remarkable difference between male and female subjects as well as between LQTS subtypes was found in our study (Chapter 4). Female LQTS patients were found to have a more pronounced QT-interval prolongation initiated by the brief tachycardia provoked by standing. Furthermore, the QT-interval prolongation was most pronounced in LQT-2 patients whereas LQT-3 patients, on average, did not show any prolongation of the QT-interval. We encourage the use of QT-interval algorithms to study the QT-interval dynamics in provocation tests since a manual assessment of the 
QT-intervals of a provocation tests offers a restrictive view of the dynamic behaviour. However, the landmarks of QRS onset and T-wave end as detected by the algorithms should be manually checked to ensure reliable QT-intervals. This is especially important for exercise and brisk standing tests since these are associated with muscle noise and baseline wander.

\subsubsection{Other QT measures}

Two more sophisticated QT measures are QT variability34-36 and QT dispersion ${ }^{37-39}$. QT variability reflects subtle temporal variations in ventricular repolarization duration and is repeatedly reported to be increased in LQTS patients ${ }^{40-42}$. The underlying physiological mechanism is not yet completely understood. At rest, variations in heart rate might be the underlying cause of QT-interval variations ${ }^{43}$. Other explanations and theories for QT-interval variations are: spontaneous sarcoplasmic reticulum calcium release; fluctuations in ion channel gating properties; and changes in sympathetic tonus ${ }^{44}$. It could very well be that all these explanations are entangled and therefore hamper the interpretation of QT variability. Besides this, the interpretation of QT variability is furthermore hampered by the diverse methods being used to determine QT variability. These methods range from the standard deviation or variance of QT-intervals over time to sophisticated measures that require frequency domain analysis of QT-intervals ${ }^{44}$. Furthermore, since QT variabilities are very small and require a lot of complexes to be measured, proper use of reliable QT-interval algorithms as well as high quality ECGs are needed to acquire a reliable QT variability. To conclude, QT variability could in future be a measure that improves LQTS diagnosis but requires more research on the mechanistic as well as the technical aspects of this measure.

Whereas QT variability is a measure for QT variations in time, QT dispersion measures the difference in QT-interval of one complex between ECG leads. The rationale behind QT dispersions is straight forward. Experimental mapping studies showed that heterogenic ventricular repolarization times were associated with ventricular arrhythmias ${ }^{45-47}$ and ECGs were believed to be capable of recording regional differences in de- and repolarization. However, the interpretation, measurement and cut-offs for QT dispersions are not as straight forward as the idea itself. Malik et al. showed that reported normal values of QT dispersion range from 10 to 71 milliseconds $^{39}$. This is supported by the broad range of QT dispersions within patients and between patients with atrial fibrillation undergoing a pulmonary vein isolation as shown in this thesis (Chapter 8). A reason for this broad range of QT dispersion might be the measurement error which, according to Malik et al., is about the size of the differences between patient groups ${ }^{39}$. Since QT dispersion is calculated as the difference between the longest and the shortest QT-interval, only one erroneous QT-interval calculation is enough for a false increased QT dispersion. However, even after manually checking all QRS onset and T-wave end landmarks in the QT dispersions mentioned in Chapter 8, the range of QT dispersions found within and between patients with atrial fibrillation was still broad. Lastly, a large QT dispersion most likely is a result of an altered T-wave morphology in at least one ECG ead. It might therefore be more useful to study the T-wave morphology itself instead of a measure that is affected by the morphology. In conclusion, though a ventricular heterogenic repolarization might lead to different QT-intervals between ECG leads, the accuracy and reliability of current QT dispersion algorithms should be investigated intensively before it can be used in the diagnosis of LQTS. Since an aberrant T-wave morphology most likely is the cause of a large QT dispersion, an intensively study to the possible added value of the T-wave morphology might be more useful than an intensive in QT dispersion.

\subsection{T-wave morphology}

Apart from a prolonged QT-interval, altered T-wave morphologies have been described in LQTS patients ${ }^{48-52}$. Many years ago, Peter Schwartz therefore already said: 'Don't (only) measure the QT-interval - look at it "153. The T-wave morphology might thus contain additional diagnostic information that is not reflected by only the QT-interval. However, the interpretation of the T-wave morphology is subjective and relies on experience. Therefore some groups developed algorithms to characterize T-wave morphology and showed an improved diagnosis when using these algorithms ${ }^{54-56}$. Despite these promising results, none of these T-wave morphology characterizations are mentioned in current international guidelines yet. In Chapters 6 and $\mathbf{7}$ we showed that two different T-wave morphology characterization methods combined with age, sex and QTc improved LQTS diagnosis in a cohort consisting of LQTS patients and LQTS family members. Though, the fact that T-wave morphology can aid in the diagnosis of LQTS is proven by now, what algorithm has the best performance is still unknown and requires a head-to-head comparison between available algorithms. Apart from the T-wave morphology at rest, Chorin et al. showed that changes in T-wave morphology increased the diagnostic value of the brisk standing test ${ }^{5}$. Therefore, apart from standard 10-second ECGs, T-wave morphology characterization algorithms should also be applied to ECGs obtained during provocation tests to study T-wave morphology dynamics. For example, applying the Hermite-Gauss polynomial T-wave morphology characterization we've described in Chapter $\mathbf{7}$ to quantify changes in T-wave morphology provoked by standing might further improve the diagnostic value of the brisk standing test. Altogether, the question to be answered is not if the T-wave morphology can aid in the diagnosis of LQTS, the question is how we can get the best out of the T-wave morphology in the diagnosis of LQTS.

\subsection{What do we want to diagnose?}

Although many efforts are made to improve LQTS diagnosis, what should be diagnosed is still not completely understood. In many studies (including Chapters 4-7 of this thesis), genetic testing results were used as the gold standard for diagnosing LQTS. Although interpreting genetic testing results seems relatively simple and straight forward, studies have shown differently. First of all, no known LQTS mutations can be found in approximately $20 \%$ of clinically diagnosed LQTS patients $5^{58-60}$. Furthermore, known pathogenic variants seem to have a reduced penetrance and therefore do not always lead to symptoms and/or a prolonged QT-interval61 ${ }^{16}$. To use genetic testing results as gold standard is therefor debatable. In Chapters 6 and 7 a sensitivity of respectively 0.82 and 0.83 was reached by combining QTc, age, sex with T-wave morphology parameters in support vector machine models. In Chapter 7 the model was validated on an external cohort and reached a sensitivity of 0.79. Figure 7-2 shows that all LQTS patients that were classified incorrectly by our model have a QTC within or slightly above the normal range. Knowing that the penetrance of LQTS gene mutations are low, these 'LQTS patients' could be patients without any phenotypic sign of LQTS and therefore classified as healthy controls by our model. The other way around, some genotype-negative family members that were incorrectly classified by our model, have prolonged QTc. These subjects might be LQTS phenotypic subjects without any known LQTS mutation. Whether this is true remains unknown but it clearly illustrates the fact that we don't know what should be diagnosed.

One could also say we should predict symptoms (for example syncope and cardiac arrest) since the reason we want to diagnose LQTS after all is to prevent symptoms regardless of genetic mutations. 
However, conducting good clinical studies to predict symptoms is very complicated because of several reasons. First of all, an asymptomatic patient can become symptomatic because of ageing or other external influences ${ }^{24}$. Therefore, susceptibility for ventricular arrhythmias might change within months, weeks, days or even within one day. How much time before the onset of symptoms should a method therefore be able to predict symptomaticity is unknown. For example, if a patient becomes symptomatic one month after it was predicted to be an asymptomatic patient, it is not clear whether the prediction was wrong or whether external influences changed and subsequently caused the symptoms. Not only does this hamper a future implication of such a model, it also hampers its development. Secondly, though preventive treatments could blur the first recognizable effects of, for example, a patient's ECG they cannot be withheld from LQTS patients since the consequences might be devastating. Lastly, symptoms very likely occur in an unmonitored setting through which the precise underlying mechanism of the symptoms remains unclear. An incidental syncope in a patient suspect for LQTS can still be a vasovagal syncope and therefore does not proof LQTS. For instance, Hofman et al. reported that 50 out of 87 subjects that experienced a syncope in a large cohort of LQTS relatives were non-carriers ${ }^{18}$. It remains unclear whether these patients should be treated as phenotype-positive or genotype-negative QTS patients. To conclude, predicting symptoms and the susceptibility to ventricular arrhythmias could be very useful but is still far away from reality. Therefore, in this thesis, genetic testing results were used as the gold standard method to diagnose LQTS.

\subsection{Future Perspectives}

The diagnosis of LQTS is hampered by many known and probably also by many unknown factors. First of all, the lack of consensus on the QT-interval measurement method and correction for heart rate methods probably are the easiest to address. What method should be the gold standard for measuring the QT-interval and correcting it for heart rate should be included in the international guidelines. We recommend using Fridericia's formula to correct QT-intervals for heart rates. The same holds for QTinterval algorithms. Currently, all ECG system manufacturers and many researchers use their ow algorithm to measure the QT-interval. The community should invest in developing and validating an open source QT-interval algorithm. Secondly, QT-interval cut-offs should be lowered and should be adjusted for sex and age. Furthermore, automatic assessment of the T-wave morphology can guide in the aid of diagnosing LQTS and should therefore be widely available. The diagnostic value of T-wave morphology dynamics during provocation tests is an undiscovered field of research and should furthermore be studied. Lastly, intensive cooperation between large LQTS centers is required to address the issues of whether symptoms or LQTS mutations should be diagnosed. A large international structured registry of all individuals suspect for LQTS containing at least standard 10-second ECGs, 24-hour Holter measurements, symptoms, genetic mutations and medication use should be performed to unrave factors that might affect LQTS diagnosis. Not only should data from more patients be gathered, studies should also focus on gathering data of individual patients more frequently. Furthermore, the use of noninvasive mapping with electrocardiographic imaging (ECGi) is a new and promising field that could aid in the diagnosis of LQTS and should be explorer further ${ }^{62}$. The use of wearables and home monitoring is currently an unexplored field of research that could aid in understanding the mechanisms behind and the temporal fluctuations in LQTS. If all this data is combined and studied carefully, hopefully, someday a PhD student will defend his/her thesis entitled 'Diagnosing LQTS, Simple'.

\subsection{References}

1. Roden, D. M. Clinical practice. Long-QT syndrome. N. Engl. . . Med. 358, 169-76 (2008).

2. Viskin, S. Long QT syndromes and torsade de pointes. Lancet 354, 1625-1633 (1999).

3. Moss, A. J., Schwartz, P. J., Crampton, R. S., Locati, E. \& Carleen, E. The long QT syndrome: a prospective international study. Circulation 71, 17-21 (1985).

4. Goldenberg, I., Moss, A. J. \& Zareba, W. QT interval: How to measure it and what is 'normal'. J. Cardiovasc. Electrophysiol. 17, 333-336 (2006).

5. Viskin, S. et al. Inaccurate electrocardiographic interpretation of long QT: The majority of physicians cannot recognize a long QT when they see one. Heart Rhythm 2, 569-574 (2005)

6. Lepeschkin, E. \& Surawicz, B. The Measurement of the Q-T Interval of the Electrocardiogram. Circulation 6, 378-388 (1952).

7. Postema, P. G., De Jong, J. S. S. G., Van der Bilt, I. A. C. \& Wilde, A. A. M. Accurate electrocardiographic assessment of the QT interval: Teach the tangent. Heart Rhythm 5, 1015-1018 (2008).

8. Vink, A. S. et al. Determination and Interpretation of the QT Interval. Circulation 138, 2345-2358 (2018).

9. Ireland, R. H., Robinson, R. T., Heller, S. R., Marques, J. L. \& Harris, N. D. Measurement of high resolution ECG QT interval during controlled euglycaemia and hypoglycaemia. Physiol. Meas. 21 295-303 (2000).

10. Kasamaki, Y. et al. Automated versus manual measurement of the QT interval and corrected QT interval. Ann. Noninvasive Electrocardiol. 16, 156-164 (2011).

11. Panicker, G. K. et al. Intra- and interreader variability in QT interval measurement by tangent and threshold methods in a central electrocardiogram laboratory. J. Electrocardiol. 42, 348-352 (2009).

12. Kligfield, P. et al. Comparison of automated measurements of electrocardiographic intervals and durations by computer-based algorithms of digital electrocardiographs. Am. Heart J. 167, 150-159. e1 (2014).

13. Vincent, G. M., Timothy, K. W., Leppert, M. \& Keating, M. The Spectrum of Symptoms and Q Intervals in Carriers of the Gene for the Long-QT Syndrome. N. Engl. J. Med. 327, 846-852 (1992).

14. Viskin, S. The QT interval: Too long, too short or just right. Heart Rhythm 6, 711-715 (2009).

15. Priori, S. G. et al. HRS/EHRA/APHRS Expert Consensus Statement on the Diagnosis and Management of Patients with Inherited Primary Arrhythmia Syndromes: Document endorsed by HRS, EHRA, and APHRS in May 2013 and by ACCF, AHA, PACES, and AEPC in June 2013. Heart Rhythm 10, 1932 1963 (2013).

16. Priori, S. G. et al. 2015 ESC Guidelines for the management of patients with ventricular arrhythmias and the prevention of sudden cardiac death the Task Force for the Management of Patients with Ventricular Arrhythmias and the Prevention of Sudden Cardiac Death of the Europea. Eur. Heart 36, 2793-2867l (2015).

17. Schwartz, P. J., Moss, A. J., Vincent, G. M. \& Crampton, R. S. Diagnostic criteria for the long QT syndrome. An update. Circulation 88, $782-784$ (1993).

18. Hofman, N. et al. Diagnostic criteria for congenital long QT syndrome in the era of molecular genetics: do we need a scoring system? Eur. Heart J. 28, 575-580 (2007).

19. Bazett, H. C. An analysis of the time-relation of electrocardiograms. Heart 7, 353-370 (1920).

20. Vandenberk, B. et al. QT correction across the heart rate spectrum, in atrial fibrillation and ventricular conduction defects. PACE - Pacing Clin. Electrophysiol. 41, 1101-1108 (2018).

21. Luo, S., Michler, K., Johnston, P. \& MacFarlane, P. W. A comparison of commonly used QT correction formulae: The effect of heart rate on the QTc of normal ECGs. J. Electrocardiol. 37, 81-90 (2004). 
22. Noordam, R. et al. Assessing prolongation of the heart rate corrected QT interval in users of tricyclic antidepressants. J. Clin. Psychopharmacol. 35, 260-265 (2015).

23. Vink, A. S., Clur, S. A. B., Wilde, A. A. M. \& Blom, N. A. Effect of age and gender on the QTc-interval in healthy individuals and patients with long-QT syndrome. Trends Cardiovasc. Med. 28, 64-75 (2018).

24. Schwartz, P. J. et al. Genotype-Phenotype Correlation in the Long-QT Syndrome. Circulation 103, 89-95 (2001).

25. Vincent, G. M., Jaiswal, D. \& Timothy, K. W. Effects of exercise on heart rate, QT, QTc and Q QS2 in the Romano-Ward inherited long QT syndrome. Am. J. Cardiol. 68, 498-503 (1991).

26. Sy, R. W. et al. Derivation and validation of a simple exercise-based algorithm for prediction of genetic testing in relatives of LQTS probands. Circulation 124, 2187-2194 (2011).

27. Horner, J. M., Horner, M. M. \& Ackerman, M. J. The diagnostic utility of recovery phase QTc durin treadmill exercise stress testing in the evaluation of long QT syndrome. Heart Rhythm 8, 16981704 (2011).

28. Chattha, I. S. et al. Utility of the recovery electrocardiogram after exercise: a novel indicator for the diagnosis and genotyping of long QT syndrome? Heart Rhythm 7, 906-911 (2010).

29. Ackerman, M. J. et al. Epinephrine-Induced QT Interval Prolongation: A Gene-Specific Paradoxical Response in Congenital Long QT Syndrome. Mayo Clin. Proc. 77, 413-421 (2002).

30. Shimizu, W. et al. Diagnostic value of epinephrine test for genotyping LQT1, LQT2, and LQT3 forms of congenital long QT syndrome. Heart Rhythm 1, 276-283 (2004).

31. Vyas, H., Hejlik, J. \& Ackerman, M. J. Epinephrine QT Stress Testing in the Evaluation of Congenital Long-QT Syndrome. Circulation 113, 1385-1392 (2006).

32. Viskin, S. et al. The Response of the QT Interval to the Brief Tachycardia Provoked by Standing. J. Am. Coll. Cardiol. 55, 1955-1961 (2010).

33. Adler, A. et al. The phenomenon of "QT stunning": The abnormal QT prolongation provoked by standing persists even as the heart rate returns to normal in patients with long QT syndrome. Heart Rhythm 9, 901-908 (2012).

34. Sarma, J. S. M., Singh, N., Schoenbaum, M. P., Venkataraman, K. \& Singh, B. N. Circadian and power spectral changes of RR and QT intervals during treatment of patients with angina pectoris with nadolol providing evidence for differential autonomic modulation of heart rate and ventricular repolarization. Am. J. Cardiol. 74, 131-136 (1994).

35. Berger, R. D. et al. Beat-to-Beat QT Interval Variability : Novel Evidence for Repolarization Lability in Ischemic and Nonischemic Dilated Cardiomyopathy. Circulation 96, 1557-1565 (1997).

36. Jensen, B. T. et al. Beat-to-Beat QT Dynamics in Healthy Subjects. Ann. Noninvasive Electrocardiol. 9, 3-11 (2004)

37. Day, C. P., McComb, J. M. \& Campbell, R. W. QT dispersion: an indication of arrhythmia risk in patients with long QT intervals. Br. Heart J. 63, 342-344 (1990).

38. Franz, M. R. \& Zabel, M. Electrophysiological basis of QT dispersion measurements. Prog. Cardiovasc. Dis. 42, 311-324 (2000)

39. Malik, M. \& Batchvarov, V. N. Measurement, interpretation and clinical potential of QT dispersion. J. Am. Coll. Cardiol. 36, 1749-1766 (2000)

40. Porta, A. et al. Autonomic Control of Heart Rate and QT Interval Variability Influences Arrhythmic Risk in Long QT Syndrome Type 1. J. Am. Coll. Cardiol. 65, 367-374 (2015).

41. Hinterseer, M. et al. Relation of Increased Short-Term Variability of QT Interval to Congenital LongQT Syndrome. Am. J. Cardiol. 103, 1244-1248 (2009).

42. Němec, J. et al. QT interval variability and adaptation to heart rate changes in patients with long QT syndrome. PACE - Pacing Clin. Electrophysiol. 32, 72-81 (2009).

43. Zaza, A., Malfatto, G. \& Schwartz, P. J. Sympathetic modulation of the relation between ventricular repolarization and cycle length. Circ. Res. 68, 1191-1203 (1991).

44. Baumert, M. et al. QT interval variability in body surface ECG: measurement, physiological basis, and clinical value: position statement and consensus guidance endorsed by the European Hear Rhythm Association jointly with the ESC Working Group on Cardiac Cellular Electroph. Europace 18, 925-944 (2016).

45. Allessie, M. A., Bonke, F. I. \& Schopman, F. J. Circus movement in rabbit atrial muscle as a mechanism of tachycardia. II. The role of nonuniform recovery of excitability in the occurrence of unidirectiona block, as studied with multiple microelectrodes. Circ. Res. 39, 168-177 (1976).

46. Han, J. \& Moe, G. K. Nonuniform Recovery of Excitability in Ventricular Muscle. Circ. Res. 14, 44-60 (1964).

47. Kuo, C. S., Munakata, K., Reddy, C. P. \& Surawicz, B. Characteristics and possible mechanism of ventricular arrhythmia dependent on the dispersion of action potential durations. Circulation 67 1356-1367 (1983)

48. Lehmann, M. H. et al. T wave 'humps' as a potential electrocardiographic marker of the long QT syndrome. J. Am. Coll. Cardiol. 24, 746-754 (1994).

49. Moss, A. J. et al. ECG T-Wave Patterns in Genetically Distinct Forms of the Hereditary Long Q Syndrome. Circulation 92, 2929-2934 (1995).

50. Dausse, E. et al. A mutation in HERG associated with notched T waves in long QT syndrome. J. Mol. Cell. Cardiol. 28, 1609-1615 (1996).

51. Yan, G.-X. \& Antzelevitch, C. Cellular basis for the normal T wave and the ECG manifestations of the long QT syndrome. J. Electrocardiol. 30, 148 (1998).

52. Zhang, L. et al. Spectrum of ST-T-Wave Patterns and Repolarization Parameters in Congenital LongQT Syndrome. Circulation 102, 2849-2855 (2000).

53. Schwartz, P.J. \& Ackerman, M. J. The long QT syndrome: A transatlantic clinical approach to diagnosis and therapy. Eur. Heart J. 34, 3109-3116 (2013).

54. Porta-Sánchez, A. et al. T-Wave Morphology Analysis in Congenital Long QT Syndrome Discriminates Patients From Healthy Individuals. JACC Clin. Electrophysiol. 3, 374-381 (2017).

55. Andersen, M. P. et al. New descriptors of T-wave morphology are independent of heart rate. J. Electrocardiol. 41, 557-561 (2008)

56. Sugrue, A. et al. Automated T-wave analysis can differentiate acquired QT prolongation from congenital long QT syndrome. Ann. Noninvasive Electrocardiol. 22, 1-7 (2017).

57. Chorin, E. et al. Diagnostic value of T-wave morphology changes during "QT stretching" in patients with long QT syndrome. Heart Rhythm 12, 2263-2271 (2015).

58. Kapplinger, J. D. et al. Spectrum and prevalence of mutations from the first 2,500 consecutive unrelated patients referred for the FAMILION@ long QT syndrome genetic test. Heart Rhythm 6 1297-1303 (2009)

59. Tester, D. J. \& Ackerman, M. J. Novel gene and mutation discovery in congenital long QT syndrome: Let's keep looking where the street lamp standeth. Heart Rhythm 5, 1282-1284 (2008).

60. Kapa, S. et al. Genetic Testing for Long-QT Syndrome. Circulation 120, 1752-1760 (2009).

61. Priori, S. G., Napolitano, C. \& Schwartz, P. J. Low penetrance in the long-QT syndrome: clinical impact. Circulation 99, 529-533 (1999).

62. Vijayakumar, R. et al. Electrophysiologic Substrate in Congenital Long QT Syndrome: Noninvasive Mapping With Electrocardiographic Imaging (ECGI). Circulation 130, 1936-1943 (2014). 


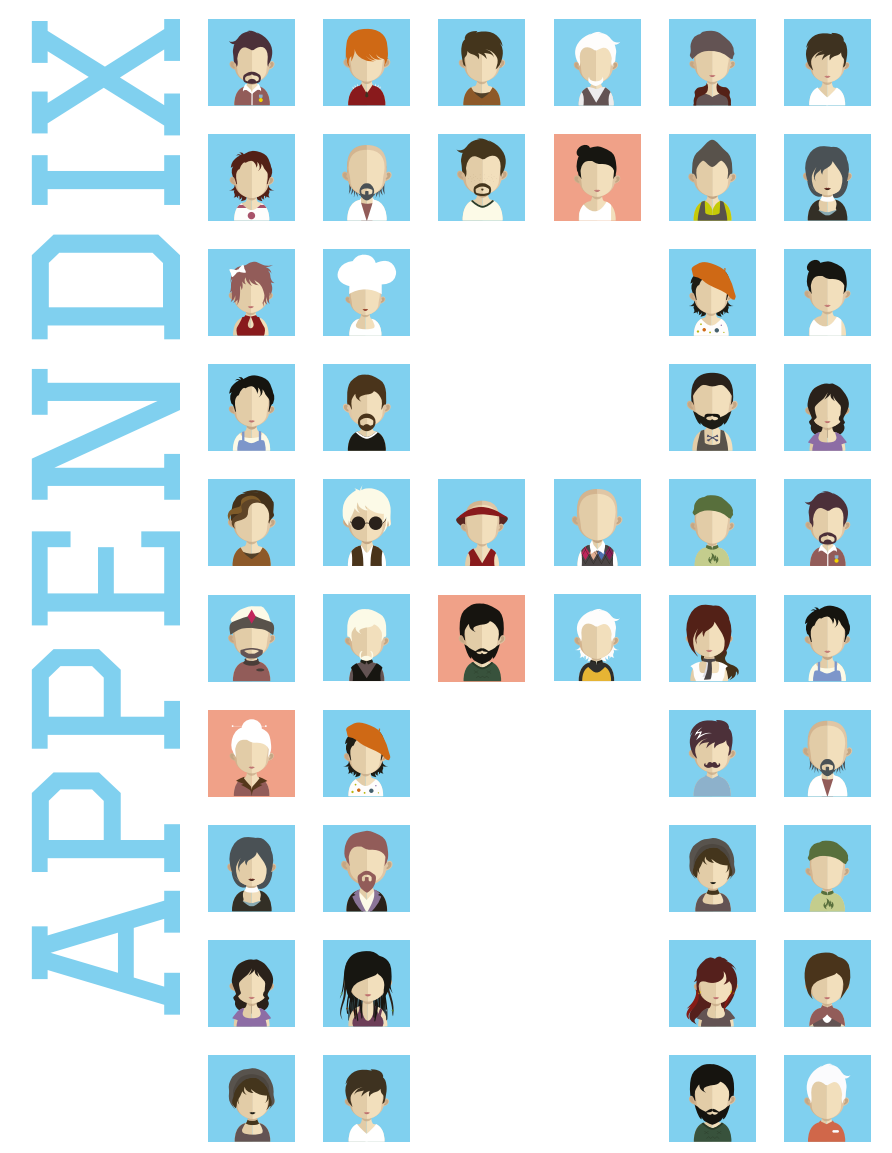

\section{APPENDIX A}

SUMMARY 
The congenital long-QT syndrome (LQTS) is an inherited condition in which the ventricular cardiac action potential can be prolonged due to an altered repolarization. The underlying mechanism of the altered repolarization is a mutation in genes encoding ventricular ion channels involved in the repolarization phase of the action potential. The prolonged action potential duration and altered repolarization is typically characterized by an increased QT-interval and/or an abnormal T-wave morphology on the electrocardiogram (ECG). LQTS patients have an increased risk on potentially lethal ventricular arrhythmias such as Torsades de Pointes. An early diagnosis is crucial since current treatments are well able to reduce the risk on a cardiac event.

Since LQTS owes its name to a prolonged QT-interval, it seems obvious to diagnose LQTS based on the QT-interval. However, diagnosing LQTS solely based on the QT-interval comes with serious limitations. First of all, manual assessment of the QT-interval is subjective and may lead to erroneous measurements. Besides that, two often used methods to manually measure the QT-interval (the threshold and tangent method) result in different QT-intervals while all maintaining the same cut-off for a prolonged QT-interval. The sensitivity and specificity for the currently used cut-offs therefore differ per method. Secondly, an existing considerable overlap in QT-intervals at rest between LQTS patients and healthy individuals hampers the diagnosis of LQTS. Therefore, the diagnostic accuracy of a QT-interval driven diagnosis is poor.

Apart from the diagnostic limitations of the QT-interval, DNA testing for known LQTS mutations also has limitations. Other (unknown) genetic factors like mutations or variants distinct from the LQTS mutation can influence the expression of the LQTS mutation or affect the repolarization via other pathways. As result, approximately $20 \%$ of phenotype-positive LQTS patients remain genetically elusive whereas on the other hand, genotype-positive LQTS patients can be phenotype-negative. Therefore, classification and reporting of potentially malign genetic variants might currently be incomplete and misleading.

In this thesis we addressed several of the above-mentioned limitations to improve the diagnosis of LQTS.

In Chapter 2, we developed and validated a QT-interval algorithm robust to heart axis orientation and T-wave morphology that can be applied on a beat-to-beat basis. The algorithm not only uses the standard 12 ECG leads, but also a root mean square, standard deviation and vectorcardiogram to measure the QT-interval from. Whereas the QRS-onset is taken from the root mean square, the end of the T-wave is defined per individual lead and per reconstructed scalar ECG leads by an automated tangent approach. The median of all T-wave ends is finally used as the general T-wave end for the particular complex. To validate the algorithm, QT-intervals measured using the algorithm were compared with the QTintervals manually measured by three observers. Measuring errors between our algorithm and manua measurements were similar or even smaller than inter-observer measuring errors and therefore we concluded that the algorithm was a good objective alternative for manual QT-interval measurements.

In Chapter 3 we investigated whether genotype-negative individuals from LQTS families have a prolonged QT-interval with respect to healthy controls. We hypothesized that, due to modifier genes, there would be a graded increase in QTc from shortest in healthy non-family members, intermediate in genotypenegative individuals from LQTS families and finally longest in genotype-positive LQTS patients. To study this hypothesis, we applied our QT-interval algorithm as described in chapter 2 to standard 10-second twelve-lead ECGs obtained from healthy subjects, genotype-negative individuals from LQTS families 
and genotype-positive LQTS patients. A multilevel linear regression analysis with QTC as the dependent variable and sex, age, and LQTS-family vs no-family and LQTS vs no-LQTS as independent variables showed that there was no difference between genotype-negative individuals from LQTS families and healthy control subjects.

In Chapters $\mathbf{4}$ and $\mathbf{5}$ we studied the brisk standing test that has been presented as a promising bedside provocation test that could aid in the diagnosis of LQTS. The so far promising results of the brisk standing test rely on manually assessed QT-intervals of several predefined complexes. In Chapters 4 and 5 we examine the diagnostic value of the QT-intervals of these complexes as well as the dynamic behaviour of the QT-intervals of all complexes in adult (Chapter 4) and paediatric (Chapter 5) LQTS patients and controls. For both the adult and the paediatric cohort, the brisk standing provocation did not result in a better classification of LQTS patients. The diagnostic accuracy of QT-intervals measured during provocation did not significantly improve compared to the diagnostic accuracy of QT-intervals during baseline (i.e. QT-intervals at rest). The reasons why our results differ from previous studies remain unknown. Assuming that the analysis protocol and the use of our QT-interval instead of manua assessment of the QT-interval did not cause these differences, inequalities in our cohort compared with the previously used cohorts might explain the differences. The data used in our study is more a reflection of a 'real-world-population' than the data used in the previous studies. For example, the control groups of the previous studies largely consisted of healthy volunteers whereas we included patients suspect for LQTS as controls.

Apart from a prolongation of the QT-interval, T-wave morphology variations are also seen in LQTS patients. In Chapters 6 and 7 we investigate the added value of T-wave morphology markers obtained from standard 10-second 12-lead ECGs in the diagnosis of LQTS. In Chapter 6 we trained and tested two models: a baseline model with age, sex, heart rate, QT-interval and QT-interval corrected for heart rate (QTc) as inputs and an extended model including several known T-wave morphology-features next to the baseline model parameters. The extended model resulted in a major rise in sensitivity and specificity compared to the baseline model. From this, we concluded that T-wave morphology does have an added value in the diagnosis of LQTS. Although the model described in Chapter 6 already increased the diagnostic accuracy, a downside of the morphology features used in this chapter is that they describe altered LQTS T-wave morphology characteristics known so far. Unrecognized altered LQTS T-wave morphology characteristics might therefore not lead to an abnormal morphology characterisation. Therefore, in Chapter 7 we developed an objective method to characterize T-wave morphology based on Hermite-Gauss polynomials. The diagnostic accuracy was again compared with a baseline mode containing age, sex and QT-intervals. The extended model again had a better overall accuracy compared to the baseline model and could, based on the T-wave morphology characterization and in contrast to the baseline model, also accurately diagnose LQTS patients with a QTc down to 400ms.

Other pathologies or treatments can also lead to a prolonged QT-interval. In Chapters 8 and 9 we studied two interventions that might lead to a prolonged QT-interval. In Chapter 8 we studied the effect of a pulmonary vein isolation (PVI) on QTc. PVI has become the cornerstone treatment for atrial fibrillation but unintentional modulation of the ganglionated plexi by PVI might affect ventricular electrophysiology. Recently, an experimental study in canine hearts showed an increased ventricular action potentia duration after ablating the ganglionated plexi. To investigate whether PVI induces a prolongation of QTc, we compared QTc's obtained one day before, one day after and three months after PVI in 279 patients. No statistically significant within-subject difference in QTc was found between the recordings indicating that PVI on average does not prolong QTc. Whether this is because a standard PVI does not modulate the ganglionated plexi (enough) or whether the gaglionated plexi modulation does not prolong QTc cannot be concluded from this study. In Chapter 9 we study the effect of various calcium concentrations in dialysates on the QT-interval. A low dialysate calcium concentration may positively affect the calcification tendency in serum of haemodialysis patients. However, calcium is an important electrolyte in the repolarization of cardiac myocytes and a lower calcium concentration in the dialysate, and subsequently in the blood, might lead to a prolonged cardiac action potential and thus a prolonged QT-interval. In Chapter 9 we analysed ECGs recorded in a four-week multicentre randomized crossover trial in which 13 patients received different dialysates during their thrice weekly haemodialysis sessions. The results of this study showed that QTc significantly increases during haemodialysis session with an acetic-acid dialysate with calcium concentration of $1.25 \mathrm{mmol} / \mathrm{L}$ and a citric-acid dialysate with a calcium concentration of $1.50 \mathrm{mmol} / \mathrm{L}$ whereas the QTC did not significantly increase during sessions with an acetic-acid dialysate with a calcium concentration of $1.50 \mathrm{mmol} / \mathrm{L}$. Benefits of the lower calcium concentration might therefore not be worthy in all haemodialysis patients and, hence, dialysate concentrations should be personalized for each patient.

In Chapter 10, all findings of the various chapters as well as all currently known limitations of the diagnosis of LQTS were put into broader perspective. The main conclusions of this thesis that could improve LQTS diagnostics are:

The automatic QT-interval algorithm described in Chapter $\mathbf{2}$ is as accurate as instructed manual observers are.

QT-intervals at rest do not differ between healthy subjects and genotype-negative individuals from LQTS families (Chapter 3).

- Contradictory with earlier studies, we could not replicate earlier documented added diagnostic value of brisk standing tests for the diagnosis of LQTS in an adult and paediatric cohort (Chapter 4 and 5)

T-wave morphology contains additional diagnostic information that can be useful to diagnose LQTS. (Chapter 6 and 7)

Pulmonary vein isolation, on average, does not induce a prolongation of QTc (Chapter 8 )

- QTC significantly increases during haemodialysis with an acetic-acid dialysate with calcium concentration of $1.25 \mathrm{mmol} / \mathrm{L}$ as well as with a citric-acid dialysate with a calcium concentration of $1.50 \mathrm{mmol} / \mathrm{L}$ (Chapter 9) 


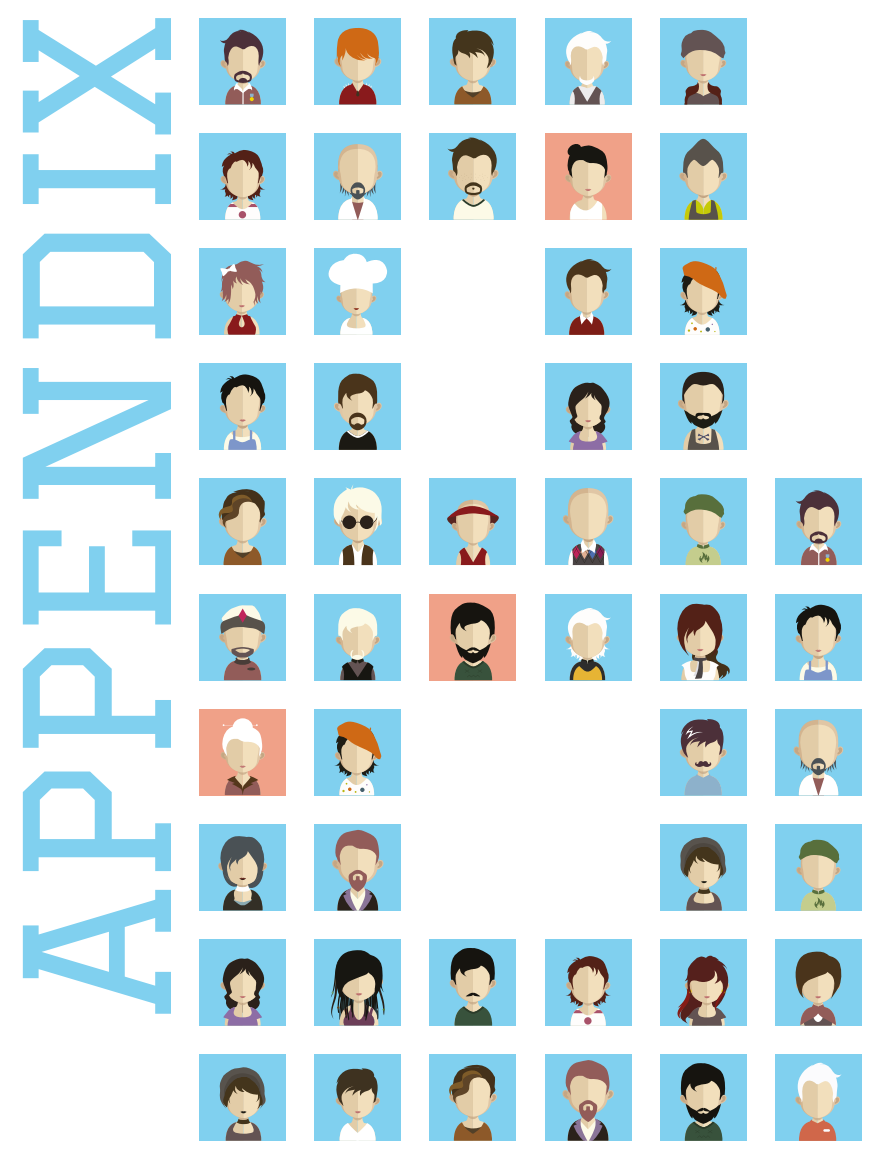

APPENDIX B

VALORISATION 


\section{B.1 Introduction}

Diagnosing the long-QT syndrome (LQTS) as soon as possible is crucial since $26 \%$ of untreated symptomatic LQTS patients will have a lethal cardiac event within three years's. If recognized and treated early enough mortality rate drops to approximately $1 \%$ over a 15-year follow-up². Unfortunately, as the title of this thesis already highlights, diagnosing LQTS is simple but not easy. One reason for this is the complex underlying genetic origin of LQTS that is not yet completely understood. For example, approximately $20 \%$ of phenotype-positive LQTS patients remain genetically elusive, i.e. none of the currently known pathogenic mutations are found in these patients ${ }^{3}$. A phenotype-based diagnosis of LQTS can also be complicated since known pathogenic mutations do not always lead to symptoms or other phenotypic signs of LQTS ${ }^{4.5}$. A considerable overlap in QT-intervals obtained from electrocardiograms (ECG) between affected and unaffected individuals6 hampers screening for LQTS based on standard ECGS.

Research into the field of diagnosing LQTS is very relevant for two reasons. First of all, new studies might directly lead to better diagnosis of LQTS because of new diagnostic tools. Secondly, studies into the field of diagnosing LQTS might lead to an enhanced understanding of the disease and the link between genotype and phenotype. In this thesis, we aimed to improve the diagnosis of genotype-positive LQTS based on computational analysis of the electrocardiogram and therefore contribute to both fields.

\section{B.2 New diagnostic tools}

In Chapter 2 we present a fully automated algorithm to measure QT-intervals. This could lead to a better diagnosis of LQTS since it has been shown that most physicians seem to be unable to identify a prolonged QT-interval when they encounter one? One of the reasons for this is erroneous QT-interva measurements. Therefore, diagnosis would be easier for physicians if they would have access to a reliable, objective and automated method to measure the QT-interval. Our algorithm is widely applicable and formed the basis of this thesis as it is applied in all follow chapters.

Apart from a prolonged QT-interval, altered T-wave morphologies can often be seen on ECG from LQTS patients and could therefore aid in the diagnosis of LQTS ${ }^{8-10}$. Many LQTS experts do not only measure the QT-interval but also evaluate the T-wave morphology in an ECG suspect for LQTS ${ }^{11,12}$. This subjective method, however, requires training and experience causing it to be more or less reserved for LQTS experts. In Chapter 6 and Chapter 7 we therefore developed two algorithm-based automatic T-wave morphology characterization methods that can help in the diagnosis of LQTS. Both algorithms had an increased accuracy in the diagnosis of LQTS compared to QT-interval driven diagnosis.

Both the automated algorithm to measure QT-intervals (Chapter 2) as well as the T-wave morphology characterizations (Chapters 6 and 7) are applicable on standard 10-second 12-lead ECGs. Since it is very cheap to record these ECGs and since they are already being recorded in all LQTS suspects, both methods can be applied without any additional costs. Especially because the algorithms are easy to reproduce or are made freely available on request by e-mail. Thereafter, since the algorithms are fully automatic, using the algorithms does not require any additional effort and physicians' experience does not affect the result. The diagnostic accuracy of LQTS could also be increased in non-specialized centres when using the algorithms presented in this thesis. Fewer patients should thereafter be referred to specialized centres for diagnosis. Apart from an improved diagnosis, the 'time-to-diagnosis' can also be 
significantly reduced by these algorithms meaning that therapies can be started earlier and patients will be kept shorter in uncertainty. The algorithms might furthermore be useful in the follow-up of LQTS patients. Changes in lifestyle, ageing or other (new) health conditions might change the expression of LQTS wherefore treatments could be reduced or should be enhanced to prevent cardiac events. The algorithms might be a useful tool to assess the expression of LQTS and therefore might lead to personalized treatments. However, this has not been investigated within this thesis, new research on this topic is needed.

\section{B.3 Enhanced understanding}

In 2010, a new elegant bed-side provocation test that could aid in the diagnosis of LQTS was introduced ${ }^{13}$. The QT-interval of LQTS patients was thought to adapt less to a short episode of tachycardia provoked by standing in comparison with healthy subjects ${ }^{13}$. In Chapter 4, we examine the diagnostic value of this test and study the dynamic behaviour of the QT-interval in a beat-to-beat manner. Up until now, the diagnostic value of the supine-standing test was promising. However, all studies were performed on an adult cohort. In Chapter 5 we examined the diagnostic value of the supine-standing test on a paediatric cohort. For both the adult (chapter 4) and the paediatric cohort (chapter 5), we remarkedly found that the QT-intervals measured during the test did not have an additional value to the QT-interval measured during baseline (which is similar to a QT-interval measured on a standard ECG). Since we were unable to reproduce the diagnostic value of the supine-standing test in adult patients and found no additional value in a paediatric cohort, the usefulness of the test suddenly is unclear. These new insights revealed that the test might lead to false positive or even worse, false negative diagnosis. As a result, healthy subjects might receive unnecessary treatment and LQTS subjects might remain untreated with all the associated consequences.

\section{B.4 References}

1. Schwartz, P. J. Idiopathic long QT syndrome: Progress and questions. Am. Heart J. 109, 399-411 (1985).

2. Schwartz, P.J. \& Crotti, L. Cardiac Electrophysiology: From Cell to Bedside. (Elsevier/Saunders, 2009).

3. Kapplinger, J. D. et al. Spectrum and prevalence of mutations from the first 2,500 consecutive unrelated patients referred for the FAMILION® long QT syndrome genetic test. Heart Rhythm 6, 1297-1303 (2009)

4. Priori, S. G., Napolitano, C. \& Schwartz, P. J. Low penetrance in the long-QT syndrome: clinical impact. Circulation 99, 529-533 (1999).

5. Crotti, L. et al. The common long-OT syndrome mutation KCNQ1/A341V causes unusually severe clinical manifestations in patients with different ethnic backgrounds: Toward a mutation-specific risk stratification. Circulation 116, 2366-2375 (2007).

6. Viskin, S. The QT interval: Too long, too short or just right. Heart Rhythm 6, 711-715 (2009).

7. Viskin, S. et al. Inaccurate electrocardiographic interpretation of long QT: The majority of physicians cannot recognize a long QT when they see one. Heart Rhythm 2, 569-574 (2005).

8. Lehmann, M. H. et al. T wave 'humps' as a potential electrocardiographic marker of the long QT syndrome. J. Am. Coll. Cardiol. 24, 746-754 (1994).

9. Moss, A. J. et al. ECG T-Wave Patterns in Genetically Distinct Forms of the Hereditary Long QT Syndrome. Circulation 92, 2929-2934 (1995)

10. Yan, G.-X. \& Antzelevitch, C. Cellular basis for the normal T wave and the ECG manifestations of the Iong QT syndrome. J. Electrocardiol. 30, 148 (1998)

11. Schwartz, P. J. Clinical significance of QT prolongation: a personal view. in Clinical Aspects of Ventricular Repolarization (eds. Butrous, G. \& Schwartz, P. J.) 343-356 (Farrand Press, 1989).

12. Schwartz, P.J. \& Ackerman, M. J. The long QT syndrome: A transatlantic clinical approach to diagnosis and therapy. Eur. Heart J. 34, 3109-3116 (2013).

13. Viskin, S. et al. The Response of the QT Interval to the Brief Tachycardia Provoked by Standing. J. Am. Coll. Cardiol. 55, 1955-1961 (2010). 


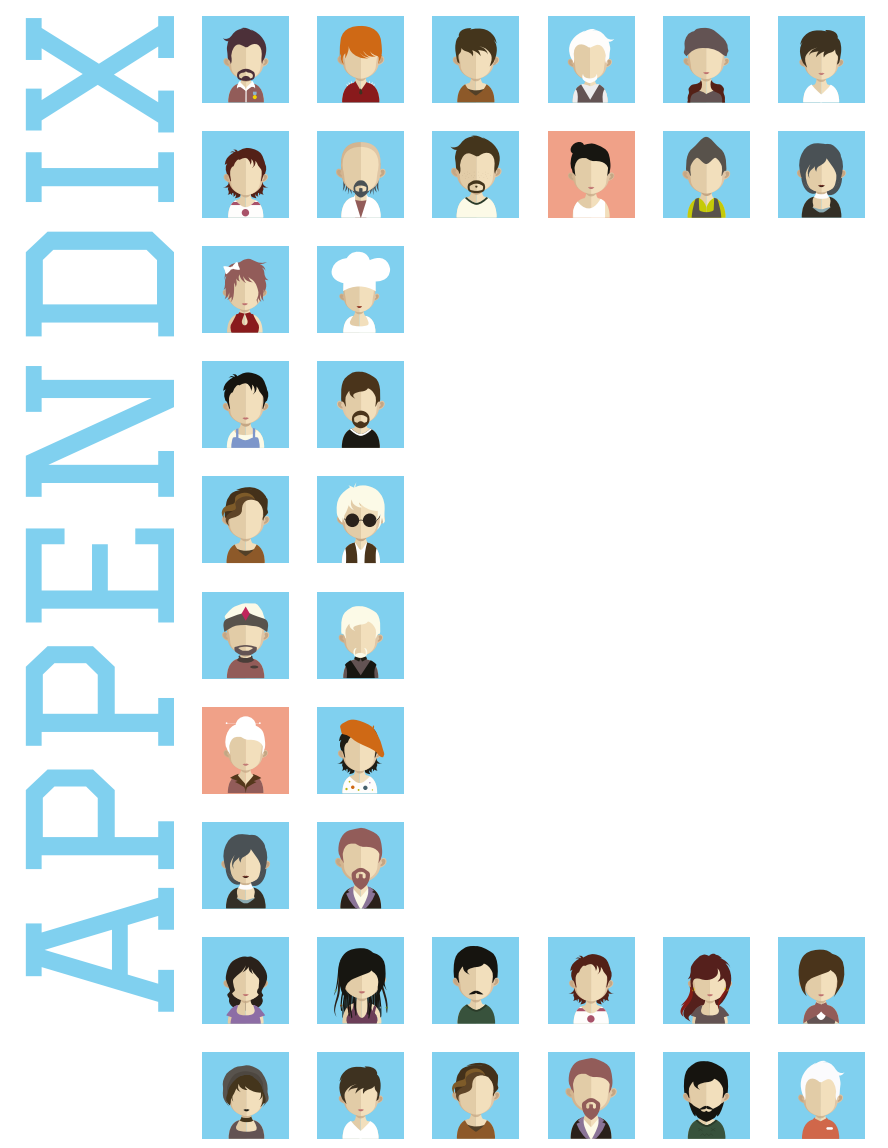

APPENDIX C

DANKWOORD / ACKNOWLEDGEMENTS 
Het moet ongeveer rond deze tijd zijn geweest, 12 jaar (!!) geleden, dat ikvoor de eerste keer bij Biomedica Engineering aan een stage begon. Toen nog voor de opleiding Biometrie. Ik besloot vervolgens zowel mijn afstudeerstage voor Biometrie als die voor Technische Geneeskunde bij BME te doen. Nu heb ik mijn laatste 'academische examen' hier ook afgerond. Ik zou dus met recht kunnen zeggen: 'de cirkel is rond'. Het is onmogelijk iedereen die mij op wat voor een manier dan ook geholpen heeft tijdens deze hele periode te bedanken. Ik richt mij daarom op de afgelopen vier jaar, dat is al moeilijk genoeg.

Uiteraard onmisbaar in deze vier jaren waren mijn promotors, prof. Delhaas en prof. Pison. Beste Tammo, jou kende ik al vanaf mijn eerste stage. Ik hoefde (in tegenstelling tot vele voorgangers, aan hun dankwoorden te lezen...) dan ook niet te wennen aan je directe commentaar. Ik heb me vanaf het begin altijd op mijn gemak bij je gevoeld. Overleg tussen ons voelde vaak als overleg tussen collega's in plaats van als overleg tussen student en leermeester of medewerker en baas. Ik heb je altijd enorm benaderbaar gevonden. Ik kon daardoor over de kleinste dingen meteen overleggen waardoor we weer op één lijn zaten en ik mijn eindsprint heb weten te voltooien. Bedankt ook voor je advies over carrièremogelijkheden. Mede dankzij jou ben ik technische geneeskunde gaan doen. Al weet jij nog steeds niet echt wat je daar mee moet.

Beste Laurent, jouw kennis over de elektrofysiologie is ongekend. Ik zal nooit vergeten hoe je meer dan eens 'Ja! Eén blik, één diagnose!' zei op de vaatkamer. Jij wist al wat er aan de hand was terwijl ik nog niet eens wist naar welke katheter ik moest kijken. Wat heb ik veel van je geleerd en wat had ik graag nog meer van je geleerd. Je bent een waar voorbeeld geweest en ik hoop iets van je kennis, maar ook zeker van je manieren (en vooral je geduld) overgenomen te hebben. Al jouw patiënten waren namelijk lovend over je. Nu ik als postdoc weer aan de slag mag met 'voorkamerfibrilleren' hoop ik je nog vaak tegen te komen!

Zeker ook onmisbaar was mijn co-promotor, dr. Postema. Beste Pieter, toen ik in het laatste jaar van mijn promotie besloot om mijn promotieonderwerp van boezemfibrilleren naar het lange QT-syndroom te veranderen kon ik echt niet zonder jou. Je was in deze periode zeer betrokken. Dat bleek niet alleen uit onze skypemeetings buiten werktijd maar ook zeker toen je in Lissabon, vlak voor mijn presentatie, op mijn schouder tikte en je een paar maand later ook in San Francisco plots naast mijn poster stond. Ik heb je inzet altijd enorm gewaardeerd en ik weet echt 100\% zeker dat dit boekje zonder jouw hulp nooit gelukt was.

Prof. Volders, prof. Blom, prof. Prinzen, prof. Vos en dr. Zeemering, leden van de beoordelingscommissie, wil ik graag hartelijk bedanken voor de kritische en vooral de snelle beoordeling van mijn proefschrift.

Ruud, het was niet altijd makkelijk in jouw voetsporen te treden. Als een echte havist betaamd, was ik namelijk een stuk minder gemotiveerd dan mijn grote broer en vond ik een zesje wel prima. Toen ik een laatbloeier bleek te zijn, is toch alles goed gekomen. Hoe mooi is het om deze dag met jou als mijn paranimf te kunnen delen.

Frank, we zijn bijna 10 jaar lang directe collega's geweest, hebben menig (ECTM-)borrel uitgespeeld en tijdens een stage zelfs een appartement in Rotterdam gedeeld. Daarnaast hebben we talloze projecten 
tijdens de studie samen met Bennis en Timon afgerond. Ons huzarenstukje moet toch wel 'Ed de GUI zijn geweest! Een logische keuze dus om jou als paranimf te vragen. Bedankt!

Suzanne ben ik enorm veel dank verschuldigd. Beste Suzanne, eerlijk is eerlijk, er zat in het begin wat uis op de lijn. Van de een op de andere dag kwamen één stel nerds uit Maastricht (of all places) jouw databases plunderen. Zo moet het ongeveer gevoeld hebben. Uiteindelijk is het allemaal wel goed gekomen (hoop ik). De hoeveelheid werk die jij verzet is buitengewoon. Inhoudelijk had iij zo maar een copromotor van me kunnen zijn. Ook de andere collega's uit Amsterdam, prof. Wilde, Veronique, Puck en Jean-Luc bedankt voor de samenwerking. Ik bewonder hoe jullie dringende wetenschappelijke vragen aanpakken, ongeacht de hoeveelheid (hand)werk die daarvoor nodig is.

Bennis, iij bent als geen ander in staat om van iets simpels iets complex te maken. Nu nog andersom. ) Bedankt voor alle hulp met machine learning. Ingewikkelde vragen tijdens mijn verdediging verwijs ik graag naar je door, dan weet ik zeker dat het uur zo om is. Ik zal een flip-over meenemen zodat je je legendarische 'Wait! 'Ill make a drawing' nog eens kunt herhalen.

Alle (ex-)collegae van BME enorm bedankt voor de super gemoedelijke en fijne sfeer op de afdeling. Voornamelijk dankzij de sfeer op de afdeling kijk ik terug op een leuke tijd. In de lunchpauzes hebbe we diverse wereldproblemen opgelost maar de AIVD-kerstpuzzels waren ons toch echt de baas. Sjeng onze origami kunstwerken en je nutteloze feitjes zal ik niet snel vergeten. Maarten, dat er door onze sportieve activiteiten op het lab niets kapot is gegaan, blijft een wonder. Je bent een werkpaard, houd dat vol maar maak het niet te bont. Erik, ouwe klusser, hoewel we er vaak over gelachen hebben, verdient het niets dan respect hoe jij promoveren en het verbouwen van een huis (in je eentje!) combineert. Peter, weinig mensen zijn zo lomp als jij. Als jij mee voetbalde waren scheenbeschermers zelfs tijdens het hooghouden geen overdreven luxe. Niek, nu je AlOS bent heeft je witte pekske eindelijk nut! Ik neem aan dat je je brieven in een combinatie van MATLAB en LaTeX maakt. Raoul, blijf oefenen met CS, misschien wordt het ooit nog wat. Aurore, merci pour les chocolats et vos conseils tout en complétant ma thèse (did absolutely not use translate for that!). Lauren, it's friday b*tch! Tijmen, bedankt voor de wekelijkse analyses over de hoogte- en dieptepunten van Oranje, Fortuna, F.C. Twente en PSV. Myrthe 'muizen-meisje', bedankt voor de gezelligheid. Je hebt nu het mooiste bureau van het lab, pas er goed op. Letty, jouw komst is de sfeer op het lab zeker ten goede gekomen. Pamir, veel succes met de afronding van jouw promotie en uiteraard veel geluk in Zitterd! Nick, bedankt dat ik jouw kamer als wachtkamer voor Tammo mocht gebruiken. Mehrdad, thank you for the 'pantoffel' football moments! Wouter, Theo en Koen, bedankt voor alle adviezen door de jaren heen. Luumes, bedankt voor alle wijze raad! Bii GVCG is het bij een talent gebleven maar ik weet zeker dat het top-talent het op de UM we gaat maken. ;) Het is aan de nieuwe(re) garde van BME, Tim, Anneloes, Lian, om de sfeer erin te houden op de afdeling. Jeroen, geniet van je pensioen! Clêhr!, bedankt voor het regelen van alle administratieve rompslomp en je interesse in huis tuin en keuken zaken en met name de schaapjes.

Uyen, Floor, Luuk, Nick, Erik, Aurore, bedankt voor de gezelligheid tijdens EHRA en HRS!

Timon, bedankt voor alle TBFF-uitjes samen met de Franken. Als mijn verdediging maar half zo goed gaat als die van jou, ben ik dik tevreden!
Alle stagiaires dieiktijdens mijn promotie heb mogen begeleiden wil ikbedanken voor de samenwerkingen ob en Sebastiaan wil ik hier extra voor bedanken. Jullie legden de basis (of nog meer dan dat) voor, respectievelijk, hoofdstuk 6 en 9 .

Hoewel het werk niet tot veel hoofdstukken in dit proefschrift heeft geleid, wil ik toch alle (EFO-) laboranten Suzanne Philippens en Matthias Zink bedanken voor de samenwerking en support. Ik zal jullie allen de komende tijd nog vaker lastigvallen!

Karlien en alle overige co-auteurs van de hoofdstukken in dit proefschrift wil ik bedanken voor de hulp en goede samenwerking.

Prof. Crijns, dr. Vernooy, Alex, Luuk en Henk, bedankt voor het actief meedenken over mijn toekomstplannen!

Leuke jongens, bedankt voor alle sjaele zeiver. Wat er ook speelde op het werk, tijdens de leuke jongens dag, een avond in de kantine of in de kelder of tijdens ons oud en nieuw uitje zorgen jullie voor de nodige afleiding. Al had ik de dag daarna meestal andere kopzorgen... Stefanie bedankt voor de tips and tricks in InDesign. Als je nu nog dingen opvallen, mag je ze voor je houden. ;

Pa en ma, om jullie te bedanken voor alle hand-en-spandiensten in en om het huis ligt wel heel erg voo de hand. Of nu een koelkast in Enschede opgehaald moest worden, een oude vijver uitgegraven moest worden of ik van of naar station moest, jullie stonden klaar. Maar ik wil jullie nu ook vooral bedanken voor het vertrouwen, de support en alle kansen die jullie mij geboden hebben. Zonder dat was ik nooit zover gekomen. Merci.

Lieve Carry, waar het verhuizen naar Sittard en Guttecoven voor mij een soort thuiskomen was, betekende dit voor jou vooral opnieuw beginnen. Ik ben zo trots op hoe jij dit gedaan hebt! Het is ech niet makkelijk om als Tukker in Limburg te integreren, maar jou is het gelukt. In de laatste periode van het promoveren heb jij veel alleen moeten doen en ben ik er niet altijd voor je geweest. Ik heb wat goed te maken. Nuup, bedankt voor je vertrouwen en support! 


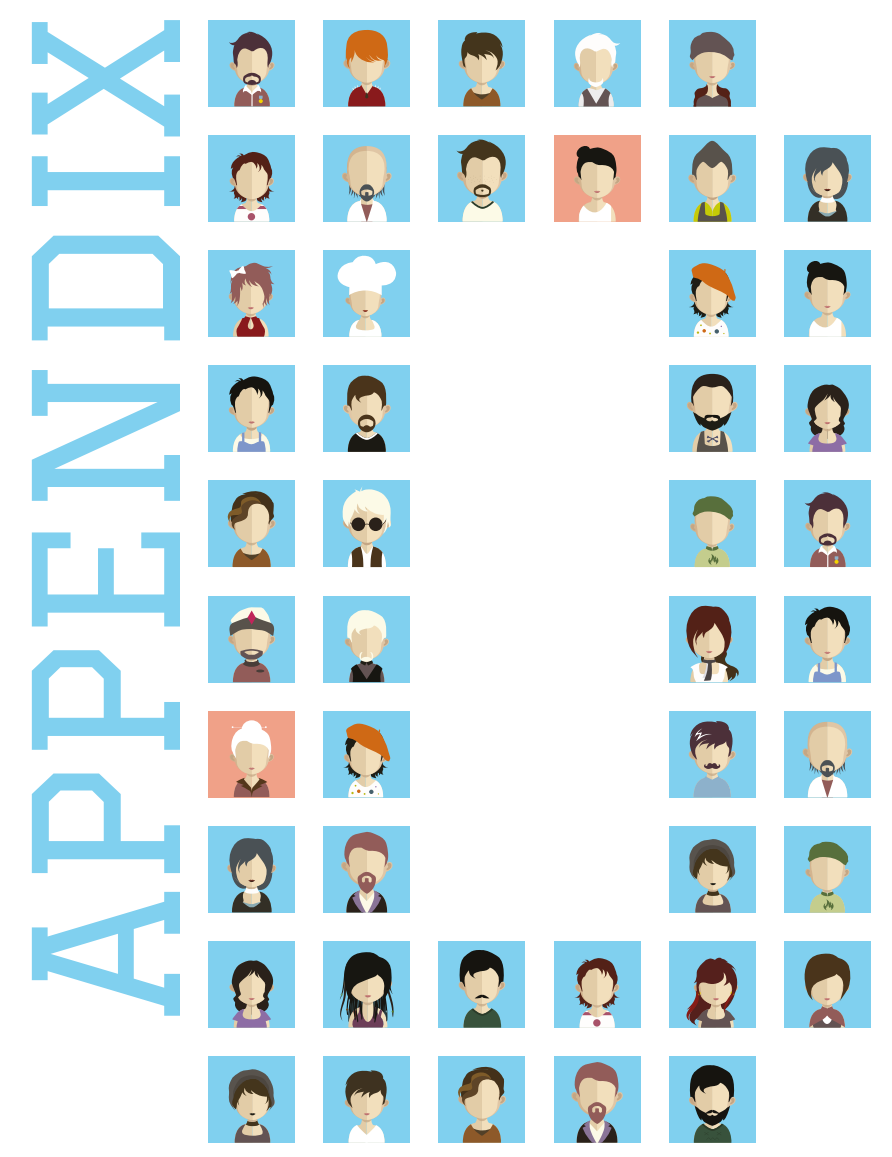

APPENDIX D

CURRICULUM VITAE 


\section{About the author}

Ben Hermans was born in Sittard in 1988 and grew up in Einighausen. In 2005 he graduated for his HAVO degree at the Trevianum in Sittard. After secondary school, Ben started to study Medical Technology (Dutch: Biometrie) at the Zuyd University for Applied Sciences. After his graduation in 2009, he started to study Technical Medicine at the University of Twente in Enschede. He obtained his Bachelor's degree for Technical Medicine in 2012 and continued to study for the Master's degree of Technical Medicine. During the three-year lasting Master track, Medical Sensing and Stimulation, he performed clinical internships at the Neonatal Intensive Care Unit in the RadboudUMC (Nijmegen), Intensive Care Unit in the Erasmus UMC (Rotterdam), general surgery department in the Meander MC (Amersfoort), and Cardiology department in the Maastricht UMC+. In 2015 he obtained his Master's degree after finishing his graduation internship at the deparments of Biomedical Engineering, Physiology and Cardiology. Ben was a student-assistant in the Experimental Centre for Technical Medicine and assisted in various bachelor courses during his study. After graduation, Ben started as a PhD candidate at the departments of Biomedical Engineering and Cardiology under supervision of prof. Delhaas and prof. Pison. He initially worked on improving the treatment of atrial fibrillation by using an advanced and new electroanatomical mapping system. Unfortunately, due to unexpected delays in the delivery of this system and high procedural costs, Ben changed his PhD project to improving the diagnosis of Long-QT Syndrome by advanced signal analysis of the electrocardiogram. Dr. Postema from the cardiology department of the Amsterdam UMC joined as a supervisor. As of November 2019, Ben works as a postdoctoral researcher at the department of Physiology under supervision of prof. Schotten. 


\section{List of publications}

- Van Geldorp IE, Delhaas T, Hermans B, et al.: Comparison of a non-invasive arterial pulse contour technique and echo Doppler aorta velocity-time integral on stroke volume changes in optimization of cardiac resynchronization therapy. Europace 2011; 13:87-95.

- $\quad$ Lansdorp B, Hermans B, Miedema H: Simulation instructor saved by student. Simul Healthc 2012; 7:391.

- Blankman P, Shono A, Hermans BJM, Wesselius T, Hasan D, Gommers D: Detection of optimal PEEP for equal distribution of tidal volume by volumetric capnography and electrical impedance tomography during decreasing levels of PEEP in post cardiac-surgery patients. Br J Anaesth 2016; 116:862-869.

- Zwanenburg A, Hermans BJM, Andriessen P, Niemarkt HJ, Jellema RK, Ophelders DR, Vullings R, Wolfs TG, Kramer BW, Delhaas T: Comparison of ECG-based physiological markers for hypoxia in a preterm ovine model. Pediatr Res IOP Publishing, 2016; 79:907-915.

- $\quad$ van't Hullenaar CDP, Hermans B, Broeders IAMJ: Ergonomic assessment of the da Vinci console in robot-assisted surgery. Innov Surg Sci 2017; 2:97-104.

- Hermans BJM, Vink AS, Bennis FC, Filippini LH, Meijborg VMF, Wilde AAM, Pison L, Postema PG, Delhaas T: The development and validation of an easy to use automatic QT-interval algorithm. Baumert M, ed: PLoS One 2017; 12:e0184352.

- Engels EB, Mafi-Rad M, Hermans BJM, Aranda A, van Stipdonk AMW, Rienstra M, Scheerder COS, Maass AH, Prinzen FW, Vernooy K: Tailoring device settings in cardiac resynchronization therapy using electrograms from pacing electrodes. EP Eur 2017; .

- $\quad$ Sathyaprabha TN, Koot LAM, Hermans BJM, Adoor M, Sinha S, Kramer BW, Raju TR, Satishchandra P, Delhaas T: Effects of Chronic Carbamazepine Treatment on the ECG in Patients with Focal Seizures. Clin Drug Investig 2018; 38.

- $\quad$ Filippini LHPM, Postema PG, Zoubin K, Hermans BJM, Blom NA, Delhaas T, Wilde AAM: The briskstanding-test for long QT syndrome in prepubertal school children: defining normal. EP Eur 2018; 20:f108-f112.

- Hermans BJM, Stoks J, Bennis FC, Vink AS, Garde A, Wilde AAM, Pison L, Postema PG, Delhaas T: Support vector machine-based assessment of the T-wave morphology improves long QT syndrome diagnosis. EP Eur 2018; 20:iii113-iii119.

- $\quad$ Andriessen P, Zwanenburg A, van Laar JOEH, Vullings R, Hermans BJM, Niemarkt HJ, Jellema RK, Ophelders DRMG, Wolfs TGAM, Kramer BW, Delhaas T: ST waveform analysis for monitoring hypoxic distress in fetal sheep after prolonged umbilical cord occlusion. Frasch MG, ed: PLoS One 2018; 13:e0195978. 